\title{
Optical and near-infrared observations of SN 2011dh - The first 100 days ${ }^{\star \star \star \star}$
}

\author{
M. Ergon ${ }^{1}$, J. Sollerman ${ }^{1}$, M. Fraser ${ }^{2}$, A. Pastorello ${ }^{3}$, S. Taubenberger ${ }^{4}$, N. Elias-Rosa ${ }^{5}$, M. Bersten ${ }^{6}$, A. Jerkstrand ${ }^{2}$,

 \\ E. Cappellaro ${ }^{3}$, M. Fiaschi ${ }^{3}$, A. Howell ${ }^{11}$, E. Kankare ${ }^{12}$, L. Magill ${ }^{2,13}$, S. Mattila ${ }^{12}$, J. Maund ${ }^{2}$, R. Naves ${ }^{15}$, P. Ochner ${ }^{3}$, \\ J. Ruiz ${ }^{14}$, K. Smith ${ }^{2}$, L. Tomasella ${ }^{3}$, and M. Turatto ${ }^{3}$
}

1 The Oskar Klein Centre, Department of Astronomy, AlbaNova, Stockholm University, 10691 Stockholm, Sweden e-mail: mattias.ergon@astro.su.se

2 Astrophysics Research Center, School of Mathematics and Physics, Queens University Belfast, Belfast, BT7 1NN, UK

3 INAF, Osservatorio Astronomico di Padova, vicolo dell'Osservatorio n. 5, 35122 Padua, Italy

${ }^{4}$ Max-Planck-Institut für Astrophysik, Karl-Schwarzschild-Str. 1, 85741 Garching, Germany

5 Institut de Ciències de l'Espai (IEEC-CSIC), Facultat de Ciències, Campus UAB, 08193 Bellaterra, Spain

${ }^{6}$ Kavli Institute for the Physics and Mathematics of the Universe (WPI), Todai Institutes for Advanced Study, University of Tokyo, 5-1-5 Kashiwanoha, Kashiwa, 277-8583 Chiba, Japan

7 INAF - Osservatorio Astronomico di Capodimonte, Salita Moiariello, 1680131 Napoli, Italy

8 Fundación Galileo Galilei-INAF, Telescopio Nazionale Galileo, Rambla José Ana Fernández Pérez 7, 38712 Breña Baja TF, Spain

9 INAF - Osservatorio Astrofisico di Catania, via Santa Sofia, 95123 Catania, Italy

10 Departamento de Ciencias Fisicas, Universidad Andres Bello, Av. Republica 252, Santiago, Chile

11 Las Cumbres Observatory Global Telescope Network, 6740 Cortona Dr., Suite 102, Goleta, CA 93117, USA

12 Finnish Centre for Astronomy with ESO (FINCA), University of Turku, Väisäläntie 20, 21500 Piikkiö, Finland

13 Isaac Newton Group, Apartado 321, 38700 Santa Cruz de La Palma, Spain

14 Observatorio Montcabrer, C Jaume Balmes 24, 08348 Cabrils, Spain

15 Observatorio de Cántabria, Ctra. de Rocamundo s/n, Valderredible, Cantabria, Spain

Received 6 May 2013 / Accepted 13 October 2013

\section{ABSTRACT}

\begin{abstract}
We present optical and near-infrared (NIR) photometry and spectroscopy of the Type IIb supernova (SN) $2011 \mathrm{dh}$ for the first 100 days. We complement our extensive dataset with Swift ultra-violet (UV) and Spitzer mid-infrared (MIR) data to build a UV to MIR bolometric lightcurve using both photometric and spectroscopic data. Hydrodynamical modelling of the SN based on this bolometric lightcurve have been presented in Bersten et al. (2012, ApJ, 757, 31). We find that the absorption minimum for the hydrogen lines is never seen below $\sim 11000 \mathrm{~km} \mathrm{~s}^{-1}$ but approaches this value as the lines get weaker. This suggests that the interface between the helium core and hydrogen rich envelope is located near this velocity in agreement with the Bersten et al. (2012) He4R270 ejecta model. Spectral modelling of the hydrogen lines using this ejecta model supports the conclusion and we find a hydrogen mass of 0.01-0.04 $M_{\odot}$ to be consistent with the observed spectral evolution. We estimate that the photosphere reaches the helium core at 5-7 days whereas the helium lines appear between $\sim 10$ and $\sim 15$ days, close to the photosphere and then move outward in velocity until $\sim 40$ days. This suggests that increasing non-thermal excitation due to decreasing optical depth for the $\gamma$-rays is driving the early evolution of these lines. The Spitzer $4.5 \mu \mathrm{m}$ band shows a significant flux excess, which we attribute to CO fundamental band emission or a thermal dust echo although further work using late time data is needed. The distance and in particular the extinction, where we use spectral modelling to put further constraints, is discussed in some detail as well as the sensitivity of the hydrodynamical modelling to errors in these quantities. We also provide and discuss pre- and post-explosion observations of the SN site which shows a reduction by $\sim 75$ percent in flux at the position of the yellow supergiant coincident with SN 2011dh. The $B, V$ and $r$ band decline rates of $0.0073,0.0090$ and $0.0053 \mathrm{mag} \mathrm{day}^{-1}$ respectively are consistent with the remaining flux being emitted by the SN. Hence we find that the star was indeed the progenitor of SN 2011dh as previously suggested by Maund et al. (2011, ApJ, 739, L37) and which is also consistent with the results from the hydrodynamical modelling.
\end{abstract}

Key words. supernovae: general - supernovae: individual: SN 2011dh - galaxies: clusters: individual: M51

\section{Introduction}

Core-collapse (CC) supernovae ( $\mathrm{SNe}$ ) are caused by the gravitational collapse of the core in massive stars. The diversity of the events that we observe reflects the diversity of the progenitor

* Figures 2, 3, Tables 3-10, and Appendices are available in electronic form at http: //www . aanda.org

$\star \star$ The photometric tables are only available at the CDS via anonymous ftp to cdsarc.u-strasbg. fr (130.79.128.5) or via http://cdsarc.u-strasbg.fr/viz-bin/qcat?J/A+A/562/A17 stars and their surrounding circumstellar media (CSM). In particular, the extent to which the star has lost its hydrogen envelope has a profound impact on the observed properties of the SN. Through the presence or absence of hydrogen lines in their spectra these $\mathrm{SNe}$ are classified as Type II or Type I, respectively. The ejecta mass of Type I SNe tends to be smaller and thus the diffusion time shorter and the expansion velocity higher. The designation $\mathrm{IIb}$ is used for $\mathrm{SNe}$ which show a spectral transition from Type II (with hydrogen) at early times to Type Ib (without 
hydrogen but with helium) at later times. These SNe are thought to arise from stars that have lost most, but not all, of their hydrogen envelope. The prime example of such a $\mathrm{SN}$ is 1993J, where the progenitor star was a yellow (extended) supergiant proposed to have lost most of its hydrogen envelope through interaction with its blue (compact) companion star (Podsiadlowski et al. 1993; Maund et al. 2004; Stancliffe \& Eldridge 2009). As Type IIb SNe are surprisingly common given the brief period single stars spend in the appropriate state, binary stars have been suggested as the main production channel - but the issue remains unresolved. Bright and nearby Type IIb SNe are rare but detection of the progenitor star in archival pre-explosion images and, when the SN has faded, a search for the companion star is feasible. By comparison of the magnitude and colour of the progenitor star to predictions from stellar evolutionary models, basic properties such as the initial mass can be estimated (Smartt et al. 2009). High quality multi-wavelength monitoring of these $\mathrm{SNe}$ followed by detailed modelling of the data is crucial to improve our understanding of Type IIb $\mathrm{SNe}$ and their progenitor stars. This paper presents the first 100 days of the extensive optical and near-infrared (NIR) dataset we have obtained for such a SN, the Type IIb 2011dh. Detailed hydrodynamical modelling of the SN using these data have been presented in Bersten et al. (2012, hereafter B12) and identification and analysis of the plausible progenitor star in Maund et al. (2011, hereafter M11). The remaining data and further modelling will be presented in forthcoming papers.

\subsection{Supernova $2011 d h$}

SN 2011dh was discovered by A. Riou on 2011 May 31.893 UT (Griga et al. 2011) in the nearby galaxy M51 at a distance of about $8 \mathrm{Mpc}$ (Sect. 1.2). The latest non-detection reported in the literature is by Palomar Transient Factory (PTF) from May 31.275 UT (Arcavi et al. 2011, hereafter A11). In this paper we adopt May 31.5 UT as the epoch of explosion and the phase of the SN will be expressed relative to this date throughout the paper.

The host galaxy M51, also known as the Whirlpool galaxy, was the first galaxy for which the spiral structure was discovered (Rosse 1850) and is frequently observed. Thus it is not surprising that excellent pre-explosion data were available in the Hubble Space Telescope (HST) archive. In M11 we used these data to identify a yellow (extended) supergiant progenitor candidate which, by comparison to stellar evolutionary models, corresponds to a star of $13 \pm 3 M_{\odot}$ initial mass. A similar analysis by Van Dyk et al. (2011) estimated an initial mass between 17 and $19 M_{\odot}$, the difference mainly stemming from the different method used to identify the evolutionary track in the HR-diagram. Recent HST (Van Dyk et al. 2013) and Nordic Optical Telescope (NOT) (Ergon et al. 2013, this paper) observations show that the yellow supergiant is now gone and indeed was the progenitor of SN $2011 \mathrm{dh}$. We discuss this issue in Sect. 5.4 and provide details of the NOT observations in Appendix B.

The SN has been extensively monitored from X-ray to radio wavelengths by several teams. Optical and NIR photometry and spectroscopy, mainly from the first 50 days, have been published by A11, M11, Tsvetkov et al. (2012, hereafter T12), Vinkó et al. (2012, hereafter V12), Marion et al. (2013, hereafter M13), Van Dyk et al. (2013, hereafter D13) and Sahu et al. (2013, hereafter S13). Radio and millimeter observations have been published by Martí-Vidal et al. (2011), Krauss et al. (2012),
Bietenholz et al. (2012), Soderberg et al. (2012) and Horesh et al. (2013) and X-ray observations by Soderberg et al. (2012), Sasaki \& Ducci (2012) and Campana \& Immler (2012). The SN has been monitored in the ultraviolet (UV) using Swift, in the mid-infrared (MIR) using Spitzer and at sub-millimeter wavelengths using Herschel. In this paper we will focus on the UV to MIR emission.

The nature of the progenitor star is an issue of great interest and there has been some debate in the literature. Using approximate models A11 argued that the SN cooled too fast and Soderberg et al. (2012) that the speed of the shock was too high to be consistent with an extended progenitor. However, in B12 we have used detailed hydrodynamical modelling to show that a 3.3-4 $M_{\odot}$ helium core with an attached thin and extended hydrogen envelope well reproduces the early photometric evolution and is also consistent with the temperature inferred from early spectra. The findings in B12 are in good agreement with those in M11 and the issue now seems to be settled by the disappearance of the yellow supergiant. See also Maeda (2012) for a discussion of the assumptions made in Soderberg et al. (2012).

The presence of a companion star (as for SN 1993J) or not is another issue of great interest. As shown in Benvenuto et al. (2013) a binary interaction scenario that reproduces the observed and modelled properties of the yellow supergiant is certainly possible. Furthermore, the prediction of a blue (compact) companion star would be possible to confirm using HST observations, preferably in the UV where the star would be at its brightest.

The paper is organized as follows. In Sects. 1.2 and 1.3 we discuss the distance and extinction, in Sect. 2 we present the observations and describe the reduction and calibration procedures, in Sect. 3 we analyse the observations and calculate the bolometric lightcurve, in Sect. 4 we compare the observations to other $\mathrm{SNe}$ and in Sect. 5 we provide a discussion, mainly related to the hydrodynamical modelling in B12 and the disappearance of the progenitor. Finally, we conclude and summarize the paper in Sect. 6. In Appendix A we provide details on the calibration of the photometry and in Appendix B we provide details on the progenitor observations.

\subsection{Distance}

In Table 1 we list all estimates for the distance to M51 we have found in the literature. As the sample is reasonably large and as it is not clear how to judge the reliability of the individual estimates we will simply use a median and the 16 and 84 percentiles to estimate the distance and the corresponding error bars. This gives a distance of $7.8_{-0.9}^{+1.1} \mathrm{Mpc}$ which we will use throughout this paper.

\subsection{Extinction}

The interstellar line-of-sight extinction towards SN 2011dh within the Milky Way as given by the extinction maps presented by Schlegel et al. (1998, hereafter S98) and recently recalibrated by Schlafly \& Finkbeiner (2011, hereafter SF11) is $E(B-V)_{\mathrm{MW}}=0.031 \mathrm{mag}$. Here and in the following the extinction within the Milky Way, the host galaxy and in total will be subscripted MW, $\mathrm{H}$ and $\mathrm{T}$ respectively and, except where otherwise stated, refer to the interstellar line-of-sight extinction towards the SN. The extinction within host galaxies is generally difficult to estimate. One class of methods used are empirical relations between the equivalent widths of the interstellar 
Table 1. Distance to M51. Literature values.

\begin{tabular}{|c|c|c|}
\hline $\begin{array}{l}\text { Distance } \\
(\mathrm{Mpc})\end{array}$ & Method & Reference \\
\hline $9.60 \pm 0.80$ & Size of HII regions & Sandage \& Tammann (1974) \\
\hline $6.91 \pm 0.67$ & Young stellar clusters & Georgiev et al. (1990) \\
\hline $8.39 \pm 0.60$ & Planetary nebula luminosity function & Feldmeier et al. (1997) \\
\hline $7.62 \pm 0.60$ & Planetary nebula luminosity function & Ciardullo et al. (2002) \\
\hline $7.66 \pm 1.01$ & Surface brightness fluctuations & Tonry et al. (2001) \\
\hline $7.59 \pm 1.02$ & Expanding photosphere method (SN 2005cs) & Takáts \& Vinkó (2006) \\
\hline $6.36 \pm 1.30$ & Type IIP SN standard candle method (SN 2005cs) & Takáts \& Vinkó (2006) \\
\hline $8.90 \pm 0.50$ & Spectral expanding photosphere method (SN 2005cs) & Dessart et al. (2008) \\
\hline 6.92 & Type Ic SN properties (SN 1994I) & Iwamoto et al. (1994) \\
\hline $7.90 \pm 0.70$ & Spectral expanding photosphere method (SN 2005cs) & Baron et al. (2007) \\
\hline $6.02 \pm 1.92$ & Spectral expanding photosphere method (SN 1994I) & Baron et al. (1996) \\
\hline 8.36 & Type IIP SN standard candle method (SN 2005cs) & Poznanski et al. (2009) \\
\hline 9.30 & Tully-Fisher relation & Tully (1988) \\
\hline $8.40 \pm 0.7$ & Expanding photosphere method (SNe 2005cs and 2011dh) & Vinkó et al. (2012) \\
\hline
\end{tabular}

Na I D absorption lines and $E(B-V)$. Relations calibrated to the extinction within other galaxies as the one by Turatto et al. (2003) are based on low resolution spectroscopy and as demonstrated by Poznanski et al. (2011) the scatter is very large. Relations based on high or medium resolution spectroscopy as the ones by Munari \& Zwitter (1997, hereafter MZ97) and Poznanski et al. (2012, hereafter P12) show a surprisingly small scatter but are calibrated to the extinction within the Milky Way. Nevertheless, given the line of sight nature of the method and the rough similarity between M51 and the Milky Way we will use these for an estimate of the extinction within M51. Ritchey $\&$ Wallerstein (2012) presented high-resolution spectroscopy of SN 2011dh resolving 8 Na I D components near the M51 recession velocity. The total widths of the $\mathrm{Na}$ I $\mathrm{D}_{2}$ and $\mathrm{D}_{1}$ lines were $180.1 \pm 5.0$ and $106.2 \pm 5.1 \mathrm{~m} \AA$ respectively. Using the MZ97 relations and summing the calculated extinction for all individual components (see discussions in MZ97 and P12) we get $E(B-V)_{\mathrm{H}}=0.05$ mag. Using the $\mathrm{P} 12$ relations for the total equivalent widths we get $E(B-V)_{\mathrm{H}}=0.03$ mag. Taking the average of these two values and adding the extinction within the Milky Way (see above) gives $E(B-V)_{\mathrm{T}}=0.07$ mag. Such a low extinction is supported by estimates from X-rays (Campana \& Immler 2012) and the progenitor spectral energy distribution (SED; M11) and we will use this value throughout the paper. The stellar population analysis done by Murphy et al. (2011) suggests a somewhat higher extinction $\left(E(B-V)_{\mathrm{T}}=0.14 \mathrm{mag}\right)$. We will adopt that value and the extinction within the Milky Way as our upper and lower error bars giving $E(B-V)_{\mathrm{T}}=0.07_{-0.04}^{+0.07} \mathrm{mag}$. Further constraints on the extinction from the SN itself and comparisons to other $\mathrm{SNe}$ is discussed in Sect. 5.1. To calculate the extinction as a function of wavelength we have used the reddening law of Cardelli et al. (1989) and $R_{V}=3.1$. For broadband photometry the extinction was calculated at the mean energy wavelength of the filters. In this paper we will consequently use the definitions from Bessell \& Murphy (2012, hereafter BM12) for the mean energy wavelength and other photometric quantities.

\section{Observations}

\subsection{Software}

Two different software packages have been used for $2 \mathrm{D}$ reductions, measurements and calibrations of the data. The IRAF based QUBA pipeline (Valenti et al. 2011, hereafter V11) and another IRAF based package developed during this work which we will refer to as the SNE pipeline. This package has been developed with the particular aim to provide the high level of automation needed for large sets of data.

\subsection{Imaging}

An extensive campaign of optical and NIR imaging was initiated for SN 2011dh shortly after discovery using a multitude of different instruments. Data have been obtained with the Liverpool Telescope (LT), the Nordic Optical Telescope (NOT), Telescopio Nazionale Galileo (TNG), Telescopio Carlos Sanchez (TCS), the Calar Alto $3.5 \mathrm{~m}$ and $2.2 \mathrm{~m}$ telescopes, the Faulkes Telescope North (FTN), the Asiago 67/92 cm Schmidt and $1.82 \mathrm{~m}$ Copernico telescopes, the William Herschel Telescope (WHT), the Large Binocular Telescope (LBT) and Telescopi Joan Oro (TJO). Amateur observations obtained at the Cantabria and Montcabrer observatories have also been included. The major contributors were the LT, the NOT, the TCS and the TNG. The dataset includes 85 epochs of optical imaging and 23 epochs of NIR imaging for the first 100 days and have been obtained thanks to a broad collaboration of European observers.

\subsubsection{Reductions and calibration}

The optical raw data were reduced with the QUBA pipeline except for the LT data for which the automatic telescope pipeline reductions have been used.

The NIR raw data were reduced with the SNE pipeline except for the United Kingdom Infrared Telescope (UKIRT) data for which the reductions provided by the Cambridge Astronomical Survey Unit (CASU) have been used. Except for the standard procedures the pipeline has support for second pass sky subtraction using an object mask, correction for field distortion and unsharp masking. Correction for field distortion is necessary to allow co-addition of images with large dithering shifts and has been applied to the TNG data. Unsharp masking removes large scales structures (e.g. the host galaxy) in the images to facilitate the construction of a master sky in the case of large scale structure overlap. Given the (usually) small fields of view and the large size of the host galaxy this technique has been applied to all data where separate sky frames were not obtained.

Photometry was performed with the SNE pipeline. We have used aperture photometry on the reference stars as well as the SN 
using a relatively small aperture (1.5-2.0 times the full width at half maximum). A mild ( $>0.1 \mathrm{mag}$ error) rejection of the reference stars as well as a mild $(3 \sigma)$ rejection of the calculated zero points were also used. Both measurement and calibration errors were propagated using standard formulae. To ensure that the photometry is free from background contamination we have, as a test, template-subtracted the NOT and LT data sets using a HOTPANTS $^{1}$ based tool provided by the SNE pipeline and latetime ( 200 days) SN subtracted images. The contamination was negligible in all bands which is not surprising as the $\mathrm{SN}$ is still bright compared to the background at $\sim 100$ days.

The optical and NIR photometry was calibrated to the Johnson-Cousins (JC), Sloan Digital Sky Survey (SDSS) and 2 Micron All Sky Survey (2MASS) systems using reference stars in the SN field in turn calibrated using standard fields. The calibration procedure is described in detail in Appendix A where we also discuss the related uncertainties. The photometry was transformed to the standard systems using S-corrections (Stritzinger et al. 2002) except for the JC $U$ and SDSS $u$ bands which were transformed using linear colour-terms. We find the calibration to be accurate to within five percent in all bands, except for the early ( $0-40$ days) NOT $U$ band observations, which show a systematic offset of $\sim 20$ percent, possibly due to the lack of S-corrections in this band. Comparisons to S-corrected Swift JC photometry as well as the photometry published in A11, V12, T12, M13, D13 and S13 supports this conclusion although some datasets show differences in the 15-30 percent range in some bands. Note that we have used JC-like $U B V R I$ filters and SDSSlike $g z$ filters at NOT whereas we have used JC-like $B V$ filters and SDSS-like ugriz filters at LT and FTN. The JC-like URI and SDSS-like uri photometry were then tied to both the JC and SDSS systems to produce full sets of JC and SDSS photometry.

\subsubsection{Space telescope observations}

We have also performed photometry on the Spitzer 3.6 and $4.5 \mu \mathrm{m}$ imaging $^{2}$ and the Swift optical and UV imaging.

For the Spitzer imaging we performed aperture photometry using the SNE pipeline and the zero points and standard aperture provided in the IRAC Instrument Handbook to calculate magnitudes in the natural (energy flux based) Vega system of IRAC. The Spitzer images were template subtracted using a HOTPANTS based tool provided by the SNE pipeline and templates constructed from archive images. Comparing with photometry on the original images, the background contamination was less than five percent in all bands.

For the Swift imaging we performed aperture photometry using the UVOTSOURCE tool provided by the HEASOFT package and the standard aperture of 5 arcsec to calculate magnitudes in the natural (photon count based) Vega system of UVOT. The optical observations were also transformed to the JC system by the use of S-corrections (Appendix A). The observations were combined using the UVOTIMSUM tool provided by the HEASOFT package and after day 5 the UV observations were subsequently combined in sequences of three to increase the signal-to-noise ratio (SNR). The Swift UV images were template subtracted using a HOTPANTS based tool provided by the SNE pipeline and templates constructed from archive images (UVW1) and SN subtracted late-time ( $~ 80$ days) images (UVM2 and $U V W 2$ ). Comparing with photometry on the original images,

\footnotetext{
1 http://www . astro.washington.edu/users/becker/ hotpants.html

2 Obtained through the DDT program by G. Helou.
}

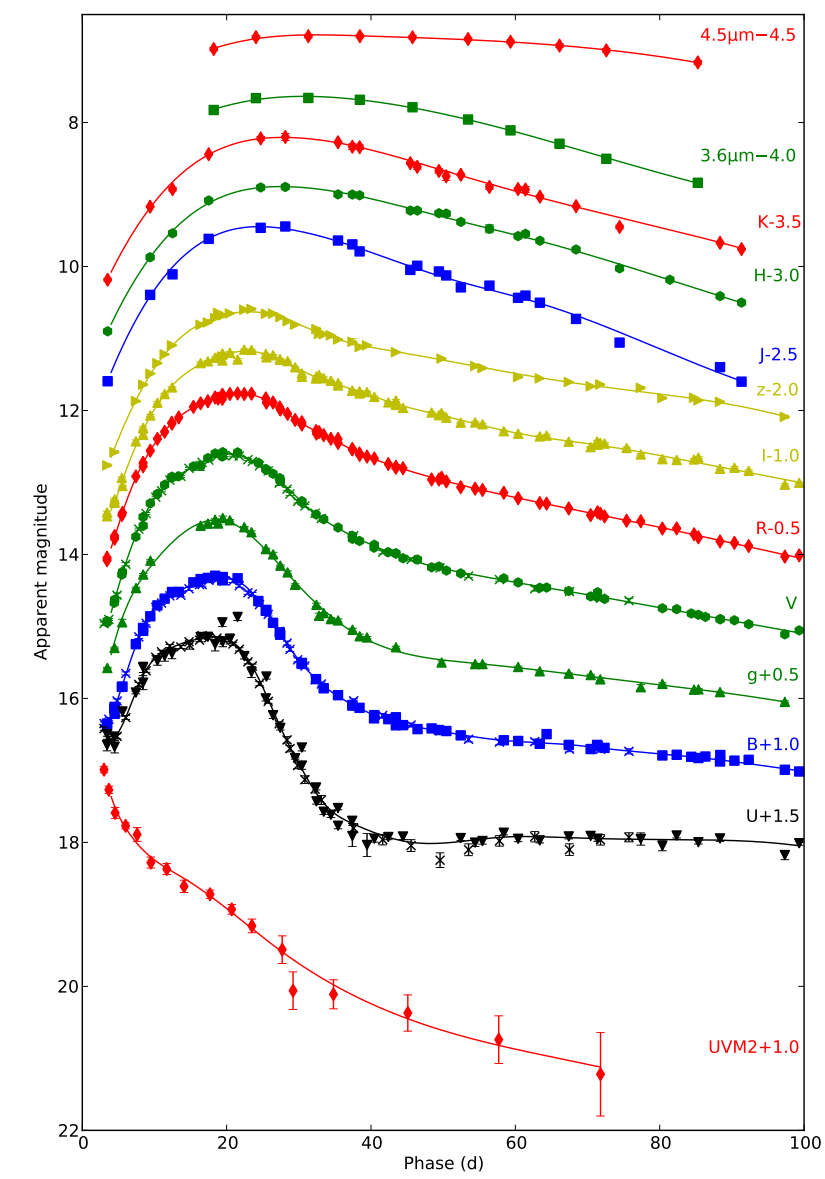

Fig. 1. Photometric evolution of SN 2011dh in the UV, optical, NIR and MIR. For clarity each band has been shifted in magnitude. Each lightcurve has been annotated with the name of the band and the shift applied. We also show the S-corrected Swift JC photometry (crosses) and cubic spline fits (solid lines).

the background contamination was negligible in the $U V W 1$ band whereas the $U V M 2$ and $U V W 2$ bands were severely affected, differing by more than a magnitude at late times. Our Swift photometry is in good agreement with that published in M13 except in the $U V M 2$ and $U V W 2$ bands after $\sim 10$ days, which is expected since M13 did not perform template subtraction. Our Swift photometry is also in good agreement with that published in A11 (given in the natural $\mathrm{AB}$ system of UVOT) except in the UVM2 and $U V W 2$ bands after $\sim 30$ days, the differences probably arising from differences in the template subtraction.

\subsubsection{Results}

The S-corrected optical (including Swift JC) and NIR magnitudes and their corresponding errors are listed in Tables 3-6 and the JC $U B V R I$, SDSS $g z$ and 2MASS $J H K$ magnitudes shown in Fig. 1. The Spitzer 3.6 and $4.5 \mu \mathrm{m}$ magnitudes and their corresponding errors are listed in Table 7 and shown in Fig. 1. The Swift UV magnitudes and their corresponding errors are listed in Table 8 and the Swift UVM2 magnitudes shown in Fig. 1. As discussed in Appendix A, because of the red tail of the filters and the strong blueward slope of the SN spectrum, the $U V W 1$ and $U V W 2$ lightcurves do not reflect the evolution of the spectrum at their mean energy wavelengths. These bands will therefore be excluded from any subsequent discussion and the calculation of the bolometric lightcurve in Sect. 3.3. Figure 1 also shows cubic spline fits using $3-5$ point knot separation, error weighting and 
a 5 percent error floor. The standard deviation around the fitted splines is less than 5 percent and mostly less than a few percent except for the Swift UVM2 band for which the standard deviation is between 5 and 10 percent on the tail. All calculations in Sect. 3 , including the bolometric lightcurve, are based on these spline fits. In these calculations the errors have been estimated as the standard deviation around the fitted splines and then propagated.

\subsection{Spectroscopy}

An extensive campaign of optical and NIR spectroscopic observations was initiated for SN 2011dh shortly after discovery with data obtained from a multitude of telescopes. Data have been obtained with the NOT, the TNG, the WHT, the Calar Alto $2.2 \mathrm{~m}$ telescope, the Asiago $1.82 \mathrm{~m}$ Copernico and $1.22 \mathrm{~m}$ Galileo telescopes and the LBT. The major contributors were the NOT and the TNG. Details of all spectroscopic observations, the telescope and instrument used, epoch and instrument characteristics are given in Table 9. The dataset includes 55 optical spectra obtained at 26 epochs and 18 NIR spectra obtained at 10 epochs for the first 100 days.

\subsubsection{Reductions and calibration}

The optical and NIR raw data were reduced using the QUBA and SNE pipelines respectively. Flats for NOT Grisms 4 and 5 were spatially shifted, typically by one pixel, to minimize the fringing in the reduced data.

The flux of optical and NIR spectra was extracted using the QUBA and SNE pipelines respectively. A large aperture and error weighting was used to reduce the wavelength dependent effect on the size of the point spread function (PSF) in the spatial direction. No corrections were done for this effect in the dispersion direction. The slit was always (initially) vertically aligned so the position of the PSF in the dispersion direction should not vary much.

The optical spectra were flux calibrated using the QUBA pipeline. A sensitivity function was derived using a spectroscopic standard star and corrected for the relative atmospheric extinction using tabulated values for each site. Telluric absorption was removed using a normalized absorption profile derived from the standard star. The significant second order contamination present in NOT Grism 4 spectra was corrected for using the method presented in Stanishev (2007). The optical spectra were wavelength calibrated using arc lamp spectra and crosscorrelated and shifted to match sky-lines.

The NIR spectra were flux calibrated and the telluric absorption removed with the SNE pipeline. A sensitivity function was derived using solar or Vega analogue standard stars selected from the Hipparcos catalogue and spectra of the sun and Vega. The interstellar extinction of the standards have been estimated from Hipparcos $B V$ photometry and corrected for when necessary. The NIR spectra were wavelength calibrated using arc lamp spectra and cross-correlated and shifted to match sky-lines.

Finally, the absolute flux scale of all spectra has been calibrated against interpolated photometry using a least square fit to all bands for which the mean energy wavelength is at least half an equivalent width within the spectral range.

\subsubsection{Results}

All reduced, extracted and calibrated spectra are available for download from the Weizmann Interactive Supernova data
REPository $^{3}$ (WISeREP) (Yaron \& Gal-Yam 2012). Figure 3 shows the sequence of observed spectra where those obtained on the same night using the same telescope and instrument have been combined. For clarity, and as is motivated by the frequent sampling of spectra, all subsequent figures in this and the following sections are based on time-interpolations of the spectral sequence. Interpolated spectra separated more than half the sampling time from observed spectra are displayed in shaded colour and should be taken with some care whereas interpolated spectra displayed in full colour are usually more or less indistinguishable from observed spectra. To further visualize the evolution, the spectra have been aligned to a time axis at the right border of the panels. The interpolations were done as follows. First all spectra were re-sampled to a common wavelength dispersion. Then, for each interpolation epoch the spectra closest in time before and after the epoch were identified resulting in one or more wavelength ranges and associated pre- and post-epoch spectra. The pre- and post-epoch spectra were then flux-calibrated with the magnitudes of the interpolation epoch, log-linearly interpolated and finally smoothly joined using a $500 \AA$ overlap range. Spectra interpolated using this method were also used in the calculations of the bolometric lightcurve (Sect. 3.3) and S-corrections (Appendix A). Figure 2 shows the interpolated optical and NIR spectral evolution of SN 2011dh for days 5-100 with a 5 day sampling. All spectra in this and subsequent figures have been corrected for redshift and interstellar extinction.

\section{Analysis}

\subsection{Photometric evolution}

Absolute magnitudes were calculated as $M_{i}=m_{i}-\mu-A_{i}$, where $m_{i}$ is the apparent magnitude in band $i, \mu$ the distance modulus and $A_{i}$ the interstellar absorption at the mean energy wavelength of band $i$. The systematic errors stemming from this approximation (as determined from synthetic photometry) is less than a few percent and can be safely ignored. The systematic errors stemming from the uncertainty in distance (Sect. 1.2) and extinction (Sect. 1.3) on the other hand are at the 30 percent level and this should be kept in mind in the subsequent discussions. All bands except the Swift UVM2 band show a similar evolution (the Spitzer MIR imaging did not start until day 20) with a strong initial increase from day 3 to the peak followed by a decrease down to a tail with a roughly linear decline rate. The maximum occurs at increasingly later times for redder bands. The drop from the maximum down to the tail is more pronounced for bluer bands and is not seen for bands redder than $z$. Both these trends are reflections of the strong decrease in temperature seen between 10 and 40 days (Fig. 5). The tail decline rates are highest for the reddest bands and almost zero for the bluest bands. It is interesting to note that the Spitzer $4.5 \mu \mathrm{m}$ band breaks this pattern and shows a markedly slower decline than the $3.6 \mu \mathrm{m}$ and the NIR bands. Warm dust or CO fundamental band emission are two possible explanations (Sect. 5.5). The times and absolute magnitudes of the maximum as well as the tail decline rates at 60 days are listed in Table 2 as measured from cubic spline fits (Fig. 1).

Early time data for the first three days have been published in A11 and T12 and show a strong decline in the $g, V$ and $R$ bands. This initial decline phase ends at about the same time as our observations begins.

http://www.weizmann.ac.il/astrophysics/wiserep/ 
Table 2. Times and absolute magnitudes of the maximum and tail decline rates at 60 days as measured from cubic spline fits.

\begin{tabular}{lccr}
\hline \hline Band & $\begin{array}{c}\text { Maximum } \\
\text { (days) }\end{array}$ & $\begin{array}{c}\text { Absolute magnitude } \\
(\mathrm{mag})\end{array}$ & $\begin{array}{r}\text { Decline rate } \\
\text { (mag day }^{-1} \text { ) }\end{array}$ \\
\hline$U V M 2$ & $\ldots$ & $\ldots$ & 0.019 \\
$U$ & 18.30 & -16.16 & -0.002 \\
$B$ & 18.88 & -16.43 & 0.007 \\
$V$ & 20.22 & -17.08 & 0.018 \\
$R$ & 21.86 & -17.37 & 0.021 \\
$I$ & 22.63 & -17.42 & 0.020 \\
$J$ & 24.45 & -17.58 & 0.027 \\
$H$ & 27.42 & -17.61 & 0.025 \\
$K$ & 27.71 & -17.78 & 0.027 \\
$3.6 \mu \mathrm{m}$ & 31.55 & -17.83 & 0.028 \\
$4.5 \mu \mathrm{m}$ & 39.62 & -18.18 & 0.009 \\
\hline
\end{tabular}

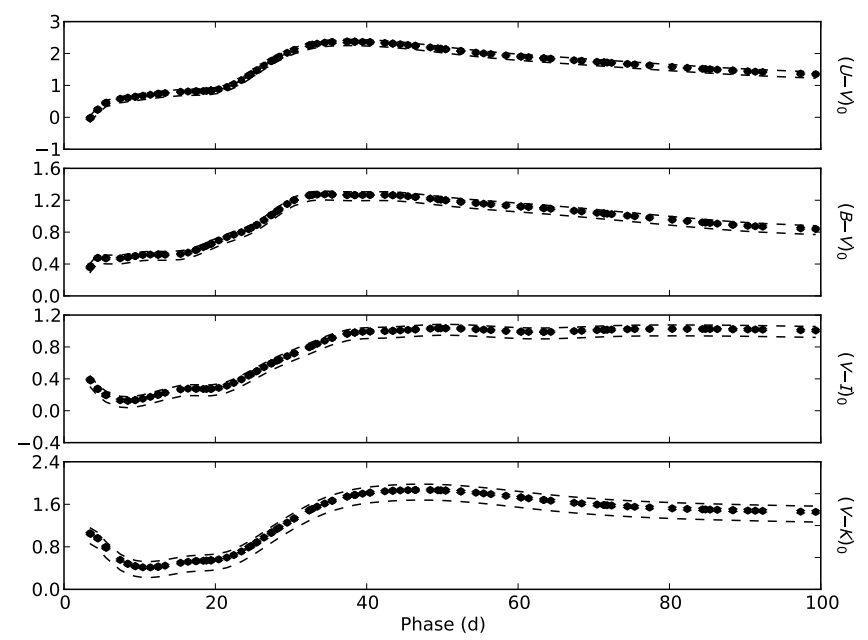

Fig. 4. $U-V, B-V, V-I$ and $V-K$ intrinsic colour evolution for SN $2011 \mathrm{dh}$ for the adopted extinction (black dots). The upper and lower error bars for the systematic error arising from extinction (black dashed lines) are also shown.

\subsection{Colour evolution and blackbody fits}

Figure 4 shows the intrinsic $U-V, B-V, V-I$ and $V-K$ colour evolution of SN 2011dh given the adopted extinction. Initially we see a quite strong blueward trend in the $V-I$ and $V-K$ colours reaching a minimum at $\sim 10$ days which is not reflected in the $U-V$ and $B-V$ colours. Subsequently all colours redden reaching a maximum at $\sim 40$ days for the $U-V$ and $B-V$ colours and $\sim 50$ days for the $V-I$ and $V-K$ colours followed by a slow blueward trend for all colours. Figures 5 and 6 show the evolution of blackbody temperature and radius as inferred from fits to the $V, I, J, H$ and $K$ bands given the adopted extinction. As discussed in Sect. 3.4, the flux in bands blueward of $V$ is strongly reduced by the line opacity in this region, in particular between 10 and 30 days. Therefore we have excluded these bands from the fits whereas the $R$ band has been excluded to avoid influence from $\mathrm{H} \alpha$ emission at early times. Note that the temperature and radius obtained correspond to the surface of thermalization rather than the photosphere (total optical depth $\sim 1$ ) and lose physical meaning when the ejecta become optically thin in the continuum. The evolution of the $V-I$ and $V-K$ colours is reflected in the evolution of the blackbody temperature, initially increasing from $\sim 7000 \mathrm{~K}$ at 3 days to a maximum of $\sim 9000 \mathrm{~K}$ at 8 days, subsequently decreasing to a minimum of $\sim 5000 \mathrm{~K}$ at $\sim 50$ days followed by a slow



Fig. 5. Evolution of the blackbody temperature for SN 2011dh as inferred from fits to the $V, I, J, H$ and $K$ bands for the adopted extinction (black dots). The upper and lower error bars for the systematic error arising from extinction (black dashed lines) and two higher extinction scenarios, $E(B-V)_{\mathrm{T}}=0.2 \mathrm{mag}\left(\right.$ red crosses) and $E(B-V)_{\mathrm{T}}=0.3 \mathrm{mag}$ (blue pluses), discussed in Sect. 5.1, are also shown.

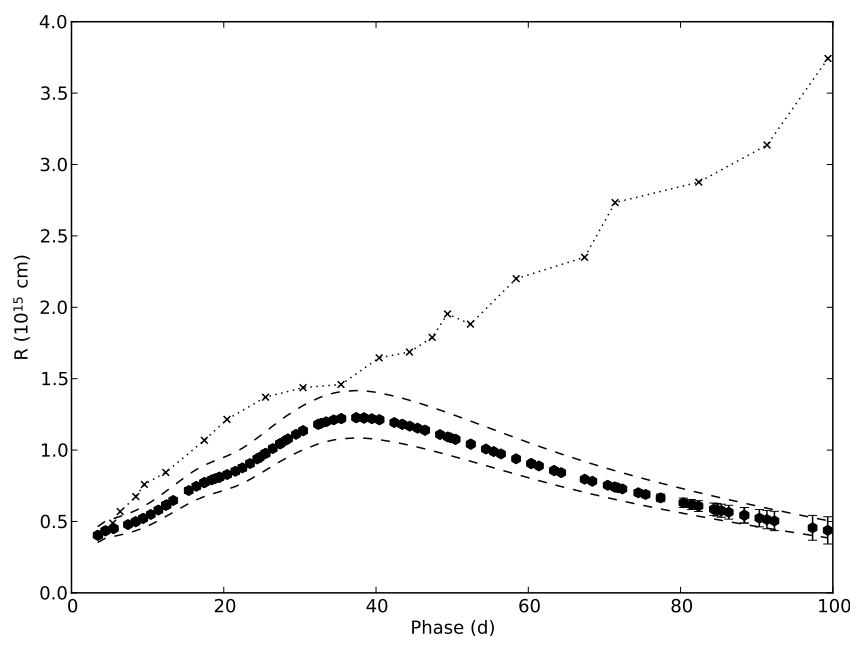

Fig. 6. Evolution of blackbody radius for SN 2011dh as inferred from fits to the $V, I, J, H$ and $K$ bands for the adopted extinction. The upper and lower error bars for the systematic error arising from extinction and distance (black dashed lines) and the radius corresponding to the P-Cygni minimum of the Fe II $5169 \AA$ line (black dotted line) are also shown.

increase. The blackbody radius shows an almost linear increase from $\sim 0.4 \times 10^{15} \mathrm{~cm}$ to a maximum of $\sim 1.2 \times 10^{15} \mathrm{~cm}$ and a subsequent almost linear decrease.

In Fig. 6 we also show the radius corresponding to the P-Cygni minimum of the Fe II $5169 \AA$ line. Interpreting this (Sect. 3.4) as the photospheric radius and the blackbody radius as the thermalization radius we see a fairly consistent evolution between 8 and 40 days corresponding to a dilution factor (ratio of photospheric and blackbody radius) increasing from $\sim 0.7$ to $\sim 0.8$ as the temperature decreases. The figure also suggests that such an interpretation breaks down for later epochs. Dilution factors for Type IIP SNe have been discussed extensively in the literature because of their importance for the EPM method (Dessart \& Hillier 2005) but are not well known for Type IIb SNe. In Fig. 7 we show dilution factors 

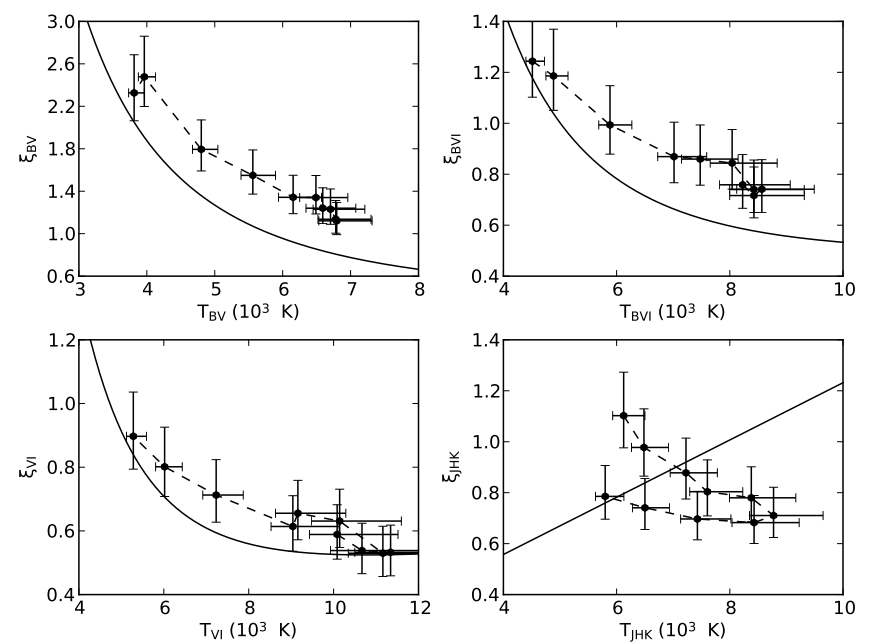

Fig. 7. Dilution factors as a function of colour temperature as inferred from blackbody fits (dots) compared to the dilution factors for Type IIP SNe determined by Dessart \& Hillier (2005) (solid lines) for the $B$ and $V$ (upper left panel), the $B, V$ and $I$ (upper right panel), the $V$ and $I$ (lower left panel) and the $J, H$ and $K$ (lower right panel) bands. In all panels we also show the upper and lower error bars for the systematic error arising from the extinction and distance.

as a function of colour temperature as inferred from blackbody fits compared to the $\xi_{B V}, \xi_{B V I}, \xi_{V I}$ and $\xi_{J H K}$ dilution factors determined for Type IIP SNe using NLTE modelling by Dessart \& Hillier (2005). The $V I$ and $J H K$ dilution factors are $\sim 10$ percent higher and $\sim 10$ percent lower on average as compared to $\xi_{V I}$ and $\xi_{J H K}$ respectively. If free-free absorption is dominating the absorptive opacity in the NIR but not in the optical, this is naively consistent with the lower charge density for helium core composition as compared to the hydrogen envelope composition of Type IIP SNe. The $B V$ and $B V I$ dilution factors are $\sim 25$ and $\sim 40$ percent higher on average as compared to $\xi_{B V I}$ and $\xi_{B V}$ respectively. The main reason for this is likely a stronger flux deficit (caused by a higher line opacity) in the $B$ band as compared to Type IIP SNe for a given thermalization temperature. V12 argue for higher values of the dilution factors as compared to Type IIP SNe because of the lower charge density and, as they point out, Baron et al. (1995) have used NLTE modelling of SN 1993J to determine a $B V$ dilution factor $\sim 60$ percent higher than for Type IIP SNe. This is similar to our (observational) result although in our interpretation this is rather due to a stronger flux deficit in the $B$ band. In the end V12 chose a value of 1.0 for their $B V R I$ dilution factor which is $\sim 10$ percent higher than our average value, the difference explained by the $\sim 10$ percent longer distance they derive. Dilution factors can never be observationally determined with better accuracy than the distance is known and NLTE modelling of Type IIb SNe is probably needed to accurately determine these. We find dilution factors involving bands redwards $B$, in particular, the $V I$ dilution factor most promising for future use in the EPM method applied to Type IIb SNe.

\subsection{Bolometric evolution}

To calculate the pseudo-bolometric lightcurve of SN 2011dh we have used a combination of two different methods. One, which we will refer to as the spectroscopic method, for wavelength regions with spectral information and one, which we will refer to as the photometric method, for wavelength regions without. The prefix pseudo here refers to the fact that a true bolometric

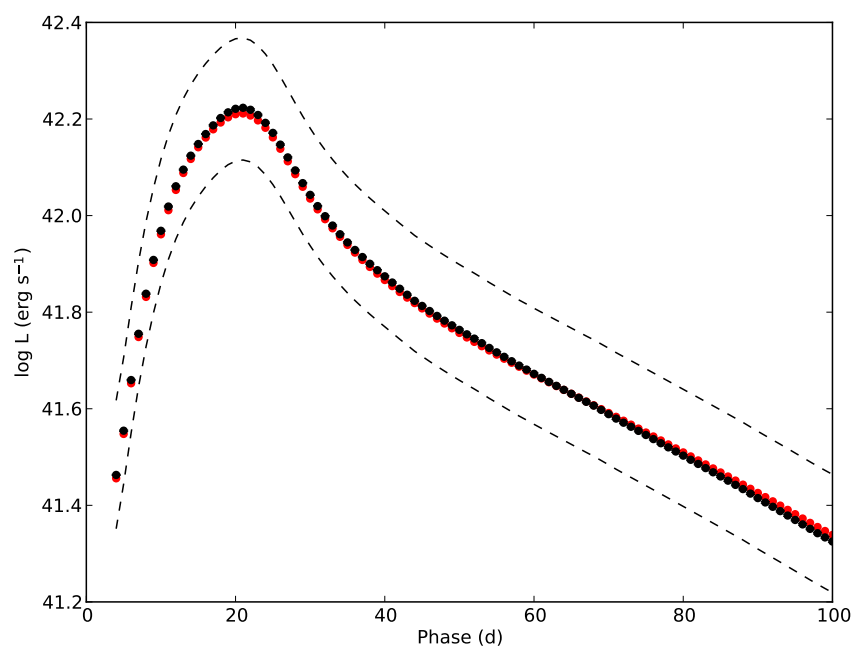

Fig. 8. Pseudo-bolometric UV to MIR lightcurve for SN 2011dh calculated with the spectroscopic (black dots) and photometric (red dots) method. The upper and lower error bars for the systematic error arising from extinction and distance (black dashed lines) are also shown.

lightcurve should be integrated over all wavelengths. We do not assume anything about the flux in wavelength regions not covered by data but discuss this issue at the end of the section.

When using the spectroscopic method we divide the wavelength region into sub-regions corresponding to each photometric band. For each epoch of photometry in each of the sub-regions a bolometric correction $B C_{i}=M_{\mathrm{bol}, i}^{\mathrm{syn}}-M_{i}^{\mathrm{syn}}$ is determined. Here $M_{i}^{\mathrm{syn}}$ and $M_{\mathrm{bol}, i}^{\mathrm{syn}}$ are the absolute and bolometric magnitudes respectively, as determined from synthetic photometry and integration of the sub-region flux per wavelength using (distance, extinction and redshift corrected) observed spectra. The bolometric magnitude in the region $M_{\mathrm{bol}}=$ $-2.5 \log \sum 10^{-0.4\left(M_{i}+B C_{i}\right)}$ is then calculated as the sum over all sub-regions, where $M_{i}$ is the absolute magnitude as determined from observed photometry. Spectra are linearly interpolated to match each epoch of photometry as described in Sect. 2.3.2. This method makes use of both spectral and photometric information and is well motivated as long as the spectral sampling is good.

When using the photometric method we log-linearly interpolate the flux per wavelength between the mean energy wavelengths of the filters. This is done under the constraint that the synthetic absolute magnitudes as determined from the interpolated SED equals the absolute magnitudes as determined from observed photometry. The solution is found by a simple iterative scheme. The total flux in the region is then calculated by integration of the interpolated flux per wavelength.

The absolute magnitudes in each band were calculated using cubic spline fits as described in Sect. 2.2.3, which is justified by the frequent sampling in all bands. When necessary, as for the Swift UV and Spitzer MIR magnitudes, extrapolations were done assuming a constant colour. The filter response functions and zeropoints used to represent the different photometric systems are discussed in Appendix A.

For SN 2011dh we have optical and NIR spectra with good sampling between 3 and 100 days and we have used the spectroscopic method in the $U$ to $K$ region and the photometric method in the UV and MIR regions. The pseudo-bolometric UV to MIR (1900-50000 ̊) lightcurve of SN 2011dh is shown in Fig. 8 and listed in Table 10 for reference. These data together with the photospheric velocity as estimated in Sect. 3.4 provide the observational basis for the hydrodynamical modelling 




Fig. 9. Fractional UV (black dots), optical (blue dots), NIR (green dots) and MIR (red dots) luminosity for SN 2011dh. The upper and lower error bars for the systematic error arising from extinction (dashed lines) and the fractional Rayleigh-Jeans luminosity redwards of $4.5 \mu \mathrm{m}$ (red solid line) are also shown. The early MIR and late UV photometry was extrapolated assuming constant colour.

of SN 2011dh presented in B12. For comparison we also show the pseudo-bolometric lightcurve calculated using the photometric method only. The difference is small but, as expected, increases slowly when the spectrum evolves to become more line dominated. The bolometric lightcurve shows the characteristics common to Type I and Type IIb SNe with a rise to peak luminosity followed by a decline phase and a subsequent tail phase with a roughly linear decline rate (Sect. 5.2). The maximum occurs at 20.9 days at a pseudo-bolometric luminosity of $16.67 \pm 0.05_{-3.68}^{+6.61} \times 10^{41} \mathrm{erg} \mathrm{s}^{-1}$, where the second error bars give the systematic error arising from the distance and extinction. The tail decline rates are 0.031, 0.021, 0.021 and $0.021 \mathrm{mag} \mathrm{day}^{-1}$ at 40,60, 80 and 100 days respectively.

Figure 9 shows the fractional luminosity in the UV (1900$3300 \AA$ ), optical (3300-10000 ̊), NIR (10 000-24000 ̊) and MIR (24 000-50000 $\AA$ ) regions respectively. The optical flux dominates and varies between $\sim 80$ and $\sim 60$ percent whereas the NIR flux varies between $\sim 15$ and $\sim 30$ percent. The UV flux initially amounts to $\sim 10$ percent, decreasing to $\sim 1$ percent at the beginning of the tail and onwards. The MIR flux initially amounts to $\sim 1$ percent, increasing to $\sim 5$ percent at the beginning of the tail and onwards. The evolution of the fractional luminosities mainly reflects the evolution of the temperature (Fig. 5) although we expect the UV to be quite sensitive to the evolution of the line opacity (Sect. 3.4).

Figure 10 shows the evolution of the SED as calculated with the photometric method overplotted with the blackbody fits discussed in Sect. 3.2 as well as the observed spectra interpolated as described in Sect. 2.3.2. The strong blueward slope in the UV region (except for the first few days) suggests that the flux bluewards of the $U V M 2$ band is negligible. The flux redwards of $4.5 \mu \mathrm{m}$ could be approximated with a RayleighJeans tail or a model spectrum. As shown in Fig. 9 the fractional Rayleigh-Jeans luminosity redwards of $4.5 \mu \mathrm{m}$ is at the percent level. Note again the excess at $4.5 \mu \mathrm{m}$ that develops between 50 and 100 days. Whereas the other bands redward of $V$ are well approximated by the blackbody fits the flux at $4.5 \mu \mathrm{m}$ is a factor of $\sim 5$ in excess at 100 days. Note also the strong reduction of

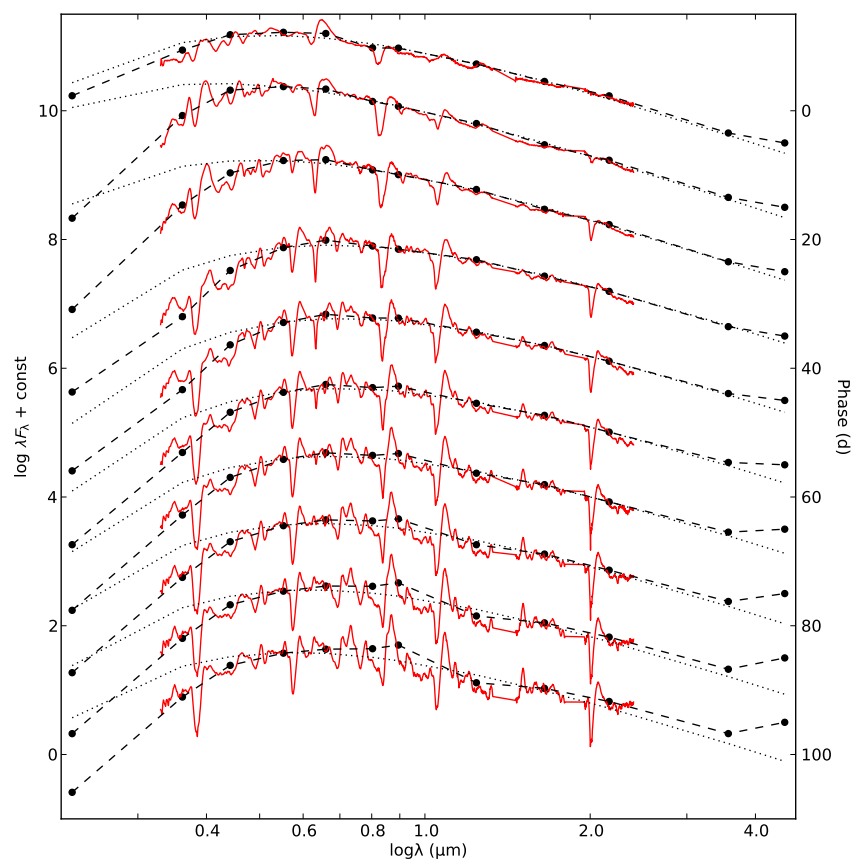

Fig. 10. Evolution of the SED as calculated with the photometric method (black dots and dashed lines) overplotted with the blackbody fits discussed in Sect. 3.2 (black dotted lines) as well as the observed spectra interpolated as described in Sect. 2.3.2 (red solid lines). The early MIR and late UV photometry was extrapolated assuming constant colour.

the flux as compared to the fitted blackbodies in bands blueward of $V$ between 10 and 30 days (Sect. 3.4).

\subsection{Spectroscopic evolution}

We have used a SN atmosphere code implementing the method presented by Mazzali \& Lucy (1993) and Abbott \& Lucy (1985) and the B12 He4R270 ejecta model with all elements except hydrogen and helium replaced with solar abundances to aid in identification of lines and some qualitative analysis of the spectra. The factor $\xi$ in Eq. (15) in Abbott \& Lucy (1985) has been set to one which might lead to overestimates of the line absorption in the optically thick limit. The Monte-Carlo based method treats line and electron scattering in the nebular approximation where the ionization fractions and level populations of bound states are determined by the radiation field approximated as a diluted blackbody parametrized by a radiation temperature. Line emission will be underestimated as the contribution from recombination is not included whereas line absorption is better reproduced. Following Mazzali \& Lucy (1993), for each epoch we have determined the temperature for the blackbody emitting surface from fits to the $V, I, J, H$ and $K$ bands and iterated the radius until the observed luminosity was achieved. Note that, except for the temperature peak between $\sim 10$ and $\sim 20$ days, the He I lines cannot be reproduced by the model as non-thermal excitation from the ground state is needed to populate the higher levels (Lucy 1991). For a quantitative analysis a NLTE-treatment solving the rate equations is necessary, in particular with respect to non-thermal excitations and ionizations. Figure 11 shows a comparison between model and observed spectra at 15 days where we also have marked the rest wavelengths of lines identified by their optical depth being $\gtrsim 1$. The atmosphere model is appropriate at early times when the approximation of a blackbody emitting surface is justified and we do not 


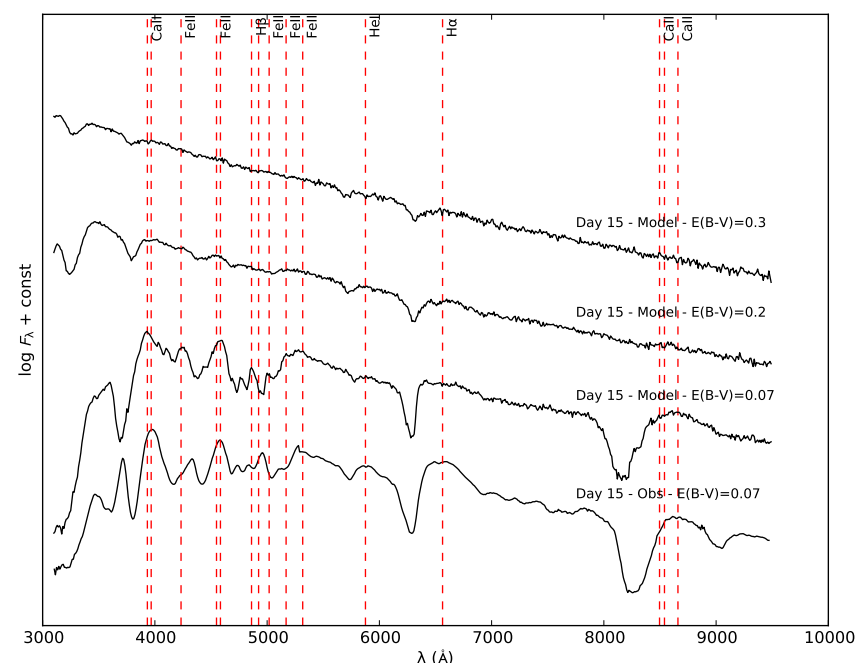

Fig. 11. Modelled and observed optical spectrum at 15 days. Lines identified by their optical depth being $\gtrsim 1$ have been marked at their rest wavelength. We also show model spectra for two higher extinction scenarios, $E(B-V)_{\mathrm{T}}=0.2 \mathrm{mag}$ and $E(B-V)_{\mathrm{T}}=0.3 \mathrm{mag}$, discussed in Sect. 5.1.

use it for phases later than $\sim 30$ days. To aid in line-identifications at later times we use preliminary results from NLTE spectral modelling of the SN spectrum at 100 days to be presented in Jerkstrand et al. (2013, in prep.). The details of this code have been presented in Jerkstrand et al. $(2011,2012)$. Both the atmosphere and NTLE code uses the same atomic data as described in these papers. The lines identified by the atmosphere modelling, the NLTE modelling or both are discussed below and have been marked in Fig. 2.

The transition of the spectra from hydrogen (Type II) to helium (Type Ib) dominated starts at $\sim 10$ days with the appearance of the He 5876 and $10830 \AA$ lines and ends at $\sim 80$ days with the disappearance of the $\mathrm{H} \alpha$ line. This transition is likely determined by the photosphere reaching the helium core, the ejecta gradually becoming optically thin to the $\gamma$-rays and eventually to the hydrogen lines. At 3 days the hydrogen signature in the spectrum is strong and we identify the Balmer series $\alpha-\gamma$, Paschen series $\alpha-\gamma$ as well as Bracket $\gamma$ using the atmosphere modelling. $\mathrm{H} \alpha$ shows a strong P-Cygni profile, extending in absorption to at least $\sim 25000 \mathrm{~km} \mathrm{~s}^{-1}$, which gradually disappears in emission but stays strong in absorption until $\sim 50$ days. Most other hydrogen lines fade rather quickly and have disappeared at $\sim 30$ days. Weak absorption in $\mathrm{H} \alpha$ and $\mathrm{H} \beta$ remains until $\sim 80$ days. Figure 12 shows closeups of the evolution centred on the hydrogen Balmer lines. Note that the absorption minimum for $\mathrm{H} \alpha$ as well as $\mathrm{H} \beta$ is never seen below $\sim 11000 \mathrm{~km} \mathrm{~s}^{-1}$ but approaches this value as the lines get weaker (see also Fig. 14). This suggests that a transition in the ejecta from helium core to hydrogen rich envelope material occurs at this velocity. Atmosphere modelling of the hydrogen lines using the B12 He4R270 ejecta model with all elements except hydrogen and helium replaced with solar abundances well reproduce the observed evolution of the absorption minima and the minimum velocity coincides with the model interface between the helium core and hydrogen rich envelope at $\sim 11500 \mathrm{~km} \mathrm{~s}^{-1}$. The good agreement with the observed minimum velocity gives further support to the B12 ejecta model. M13 estimated hydrogen to be absent below $\sim 12000 \mathrm{~km} \mathrm{~s}^{-1}$ by fitting a SYNOW (Branch et al. 2003) model spectrum to the observed spectrum at 11 days. We find the behaviour of the hydrogen lines in the weak limit to

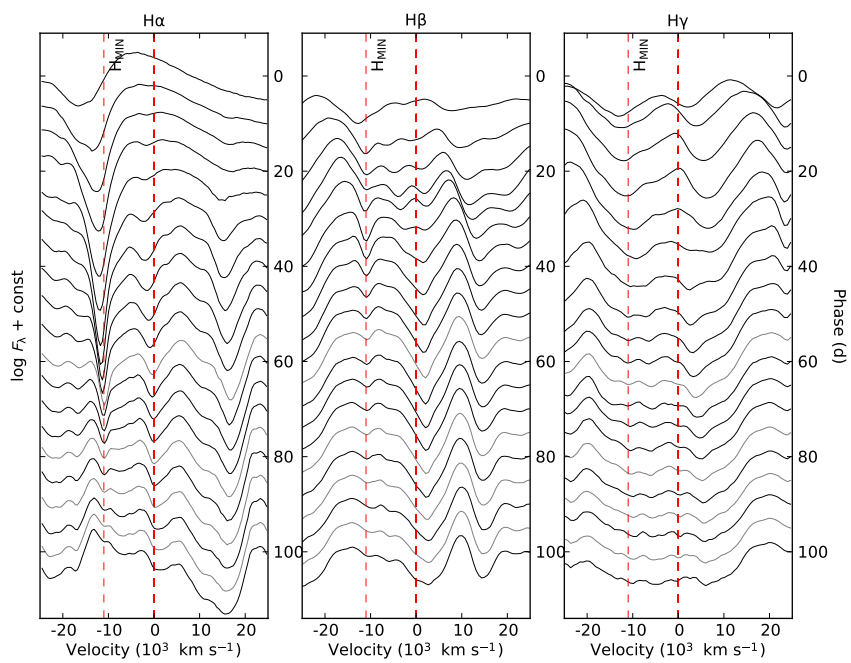

Fig. 12. Closeup of (interpolated) spectral evolution centred on the $\mathrm{H} \alpha$ (left panel), $\mathrm{H} \beta$ (middle panel) and $\mathrm{H} \gamma$ (right panel) lines. All panels in this and the following figure show the minimum velocity for the $\mathrm{H} \alpha$ absorption minimum (marked $\mathrm{H}_{\mathrm{MIN}}$ ) interpreted as the interface between the helium core and hydrogen envelope.

provide a better constraint and conclude that the interface between the helium core and hydrogen rich envelope is likely to be located at $\sim 11000 \mathrm{~km} \mathrm{~s}^{-1}$. By varying the fraction of hydrogen in the envelope we find a hydrogen mass of 0.01-0.04 $M_{\odot}$, in agreement with the $0.02 M_{\odot}$ in the original model, to be consistent with the observed evolution of the hydrogen lines. A11 used spectral modelling similar to the one in this paper, but with a NLTE treatment of hydrogen and helium, to estimate the hydrogen mass to $0.024 M_{\odot}$.

The He I lines appears in the spectra between $\sim 10$ (He I 10830 and $5876 \AA$ ) and $\sim 15$ (He I 6678, 7065 and $20581 \AA$ ) days. Later on we see the 5016 and $17002 \AA$ lines emerge as well. As mentioned the atmosphere modelling does not well reproduce the $\mathrm{He}_{\mathrm{I}}$ lines but those identified here are present in the model spectrum with optical depths of 0.1-5 during the temperature peak between $\sim 10$ and $\sim 20$ days. Increasing the He I excitation fraction to mimic the non-thermal excitation reproduce the $\mathrm{He}_{\mathrm{I}}$ lines and their relative strengths reasonably well. At 100 days, all He I lines, except He I $17002 \AA$, are present and identified by the NLTE modelling. Given the low ionization potential of $\mathrm{Na}$ I and the high temperatures we find it unlikely that $\mathrm{He}_{\text {I }} 5876$ is blended with Na I 5890/5896 at early times. Using the atmosphere modelling we find a very low ion fraction of $\mathrm{Na}$ I $\left(<10^{-7}\right)$ and the optical depth for Na I 5890/5896 to be negligible during the first 30 days. Using the NLTE modelling at 100 days we find emission to arise primarily from $\mathrm{Na}$ I 5890/5896 and absorption to be a blend. He I 10830 is likely to be blended with Paschen $\gamma$ at early times and He I $5016 \AA$ is likely to be blended with $\mathrm{Fe}_{\mathrm{I}} 5018 \AA$. Figure 13 shows a closeup of the evolution centred on the He I lines. Helium absorption is mainly seen below the $\sim 11000 \mathrm{~km} \mathrm{~s}^{-1}$ attributed to the interface between the helium core and the hydrogen rich envelope although He I $10830 \AA$ absorption extends beyond this velocity and also shows a narrow dip close to it between $\sim 30$ and $\sim 60$ days. We may speculate that this dip is caused by a denser shell of material close to the interface as was produced in explosion modelling of SN 1993J (e.g. Woosley et al. 1994). Whereas the fading and disappearance of the hydrogen lines are driven by the decreasing density and temperature of the envelope 


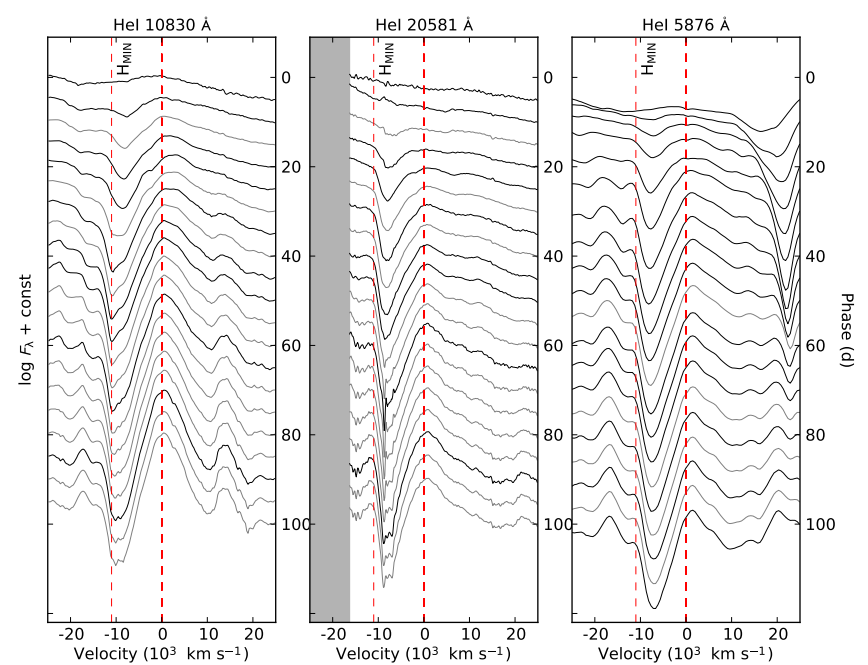

Fig. 13. Closeup of (interpolated) spectral evolution centred on the He I $10830 \AA$ (left panel), He I $20581 \AA$ (middle panel) and He I $5876 \AA$ (right panel) lines.

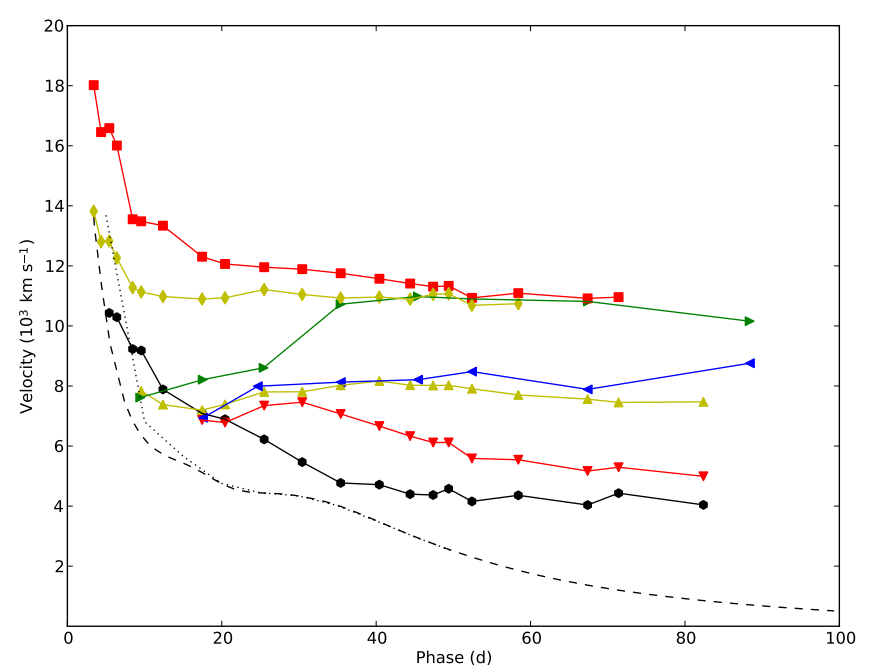

Fig. 14. Velocity evolution of the absorption minimum of the Fe II $5169 \AA$ (black circles), He I $5876 \AA$ (yellow upward triangles), He I $6678 \AA$ (red downward triangles), He I $10830 \AA$ (green rightward triangles), $\mathrm{He}_{\mathrm{r}} 20581 \AA$ (blue leftward triangles), $\mathrm{H} \alpha$ (red squares) and $\mathrm{H} \beta$ (yellow diamonds) lines as automatically measured from the spectral sequence. For comparison we also show the velocity corresponding to the blackbody radius as determined from fits to the photometry (black dashed line) and as iteratively determined by the atmosphere modelling (black dotted line).

the appearance and growth of the helium lines is likely to be more complex. M13 suggest that the helium lines appear because the photosphere reaches the helium core. However, Fig. 14 (see below) suggests that the photosphere reaches the helium core at 5-7 days whereas the helium lines appear later, at lower velocities, close to the region where we expect the continuum photosphere to be located and then move outwards in velocity until $\sim 40$ days. This rather suggest the appearance and subsequent evolution to be driven by increasing non-thermal excitation due to the decreasing optical depth for the $\gamma$-rays. For the line optical depth at a given velocity (using the Sobolev 1957 approximation) we have $\tau \propto t^{-2} x_{l}$, where $x_{l}$ is the fraction of He I in the lower state. As the temperature decreases after $\sim 10$ days and the ion fraction of $\mathrm{He}_{\mathrm{I}}$ is high according to the modelling, we would expect the line optical depth at a given velocity to decrease if non-thermal excitation was not important. Detailed modelling including a treatment of non-thermal excitation of the helium lines is needed to better understand the behaviour of the He I lines.

Except for $\mathrm{H}_{\mathrm{I}}$ and $\mathrm{He}_{\mathrm{I}}$ we also identify lines from Ca II, Fe II, OI, MgI and NaI in the spectra. The CaII 3934/3968 and 8498/8542/8662 $\AA$ lines are present throughout the evolution showing strong P-Cygni profiles and are identified by both the atmosphere and NLTE modelling whereas the [Ca II] 7291/ $7323 \AA$ line is identified by the NLTE modelling at 100 days. The O I 5577, 7774, 9263, 11300 , and $13164 \AA$ lines are all identified by the NLTE modelling at 100 days. The atmosphere modelling does not reproduce the $\mathrm{O}_{\mathrm{I}}$ lines at early times but the O I $7774 \AA$ line seems to appear already at $\sim 25$ days and the other lines between $\sim 30$ and $\sim 50$ days. The NLTE modelling also identifies the emerging [O I] 6300/6364 lines at 100 days. The Mg I $15040 \AA$ line is identified by the NLTE modelling at 100 days and seem to emerge at $\sim 40$ days. As mentioned above, we identify the Na I 5890/5896 $\AA$ lines in emission and blended in absorption with the He I $5876 \AA$ line at 100 days using the NLTE modelling. In the region 4000-5500 $\AA$, we identify numerous Fe II lines using the atmosphere modelling, the most prominent being Fe II 4233, 4549, 4584, 4924, 5018, 5169 and $5317 \AA$. These lines are present already at $\sim 5$ days and most of them persist to at least 50 days. As mentioned in Sect. 3.3 and as can be seen in Fig. 10 there is a strong reduction of the flux bluewards of $5000 \AA$ between $\sim 10$ and $\sim 30$ days. This well known behaviour, which is also reproduced by the modelling, is caused by an increased line opacity from a large number of metal ion (e.g. Fe II and $\mathrm{Cr}$ II) lines. This explains the initial redward trend in the $U-V$ and $B-V$ colours contrary to the blueward trend in $V-I$ and $V-K$ caused by the increasing temperature (see Fig. 4). Judging from Fig. 10 the reduction of the flux is considerably reduced after $\sim 30$ days.

Figure 14 shows the evolution of the absorption minimum for a number of lines as determined from the spectral sequence. These were measured by a simple automatic centring algorithm where the spectra were first smoothed down to $500 \mathrm{~km} \mathrm{~s}^{-1}$ and the absorption minimum then traced through the interpolated spectral sequence and evaluated at the dates of observation. We also show the velocity corresponding to the blackbody radius as determined from fits to the photometry and as iteratively determined by the atmosphere modelling. Because of backscattering, the model blackbody radius is larger than the fitted. It is reasonable to expect that the photosphere is located somewhere between the blackbody surface and the region where the line with the lowest velocity is formed. This line is the Fe II $5169 \AA$ line which was used in B12 to estimate the photospheric velocities. Dessart \& Hillier (2005) have used NLTE modelling to show that the absorption minimum of the Fe II $5169 \AA$ line is a good estimator for the photospheric velocity in Type IIP SNe but it is not clear that this also apply to Type IIb SNe. Thus we have to consider the possibility that the photospheric velocities could be overestimated with up to 50 percent and in Sect. 5.3 we will discuss how such an error would effect the results in B12.

\section{Comparison to other SNe}

In this section we compare the observations of SN 2011dh to the well observed Type IIb SNe 1993J and 2008ax. In order to do this we need to estimate their distance and extinction. This will 
be done without assuming similarity among the SNe and in analogy with SN 2011dh we will use high-resolution spectroscopy of the Na I D and K I 7699 A interstellar absorption lines to estimate the extinction. In the end of the section we will investigate what difference an assumption of similarity among the SNe will make.

\subsection{SN 1993J}

SN 1993J which occurred in M81 is one of the best observed $\mathrm{SNe}$ ever and the nature of this $\mathrm{SN}$ and its progenitor star is quite well understood. Shigeyama et al. (1994) and Woosley et al. (1994) used hydrodynamical modelling to show that a progenitor star with an initial mass of $12-15 M_{\odot}$ with an extended (not specified) but low mass $\left(0.2-0.9 M_{\odot}\right)$ hydrogen envelope reproduces the observed bolometric lightcurve. This was confirmed by the more detailed modelling of Blinnikov et al. (1998). Progenitor observations were presented in Maund et al. (2004) while Stancliffe \& Eldridge (2009) used stellar evolutionary models to show that a progenitor star with an initial mass of 15-17 $M_{\odot}$ with an extended but low mass hydrogen envelope, stripped through mass transfer to a companion star, reproduces the observed progenitor luminosity and effective temperature. Photometric and spectroscopic data for SN 1993J were taken from Lewis et al. (1994), Richmond et al. (1996), Matthews et al. (2002), Wada \& Ueno (1997) and IAU circulars.

The distance to M81 is well constrained by Cepheid measurements, the mean and standard deviation of all such measurements listed in the NASA/IPAC Extragalactic Database (NED) being $3.62 \pm 0.22 \mathrm{Mpc}$, which we will adopt. The extinction within the Milky Way as given by the S98 extinction maps recalibrated by SF11 is $E(B-V)_{\mathrm{MW}}=0.07 \mathrm{mag}$. Richmond et al. (1994) discuss the extinction in some detail and suggest a total $E(B-V)_{\mathrm{T}}$ between 0.08 and $0.32 \mathrm{mag}$. High-resolution spectroscopy of the Na I D lines was presented in Bowen et al. (1994). Given the rough similarity between M81 and the Milky Way we will use the MZ97 and P12 relations to estimate the extinction within M81. Bowen et al. (1994) resolve a system of components near the M81 recession velocity and another one near zero velocity. There is also a third system which the authors attribute to extragalactic dust in the M81/M82 interacting system. The individual components of all three systems are quite heavily blended. As it is not clear whether the third system belongs to the Milky Way or M81, we calculate the extinction for all the three systems with the MZ97 and P12 relations and sum to get estimates of the total extinction. The MZ97 relation gives $E(B-V)_{\mathrm{T}}=0.28 \mathrm{mag}$ and the $\mathrm{P} 12$ relations $E(B-V)_{\mathrm{T}}=0.17 \mathrm{mag}$ (on average). Given that each system clearly consists of multiple components the MZ97 relation rather provides an upper limit (see discussion in MZ97) and we will adopt the lower value given by the P12 relations. Adopting the higher value given by the MZ97 relation and the extinction within the Milky Way as upper and lower error limits we then get $E(B-V)_{\mathrm{T}}=0.17_{-0.10}^{+0.11} \mathrm{mag}$.

\subsection{SN 2008ax}

SN 2008ax is another well observed Type IIb SN but the nature of this SN and its progenitor star is not as well understood as for SN 1993J. Tsvetkov et al. (2009) used the hydrodynamical code STELLA (Blinnikov et al. 1998) to show that a progenitor star with an initial mass of $13 M_{\odot}$ with an extended $\left(600 R_{\odot}\right)$ and low mass (not specified) hydrogen envelope well reproduces the $U B V R I$ lightcurves except for the first few days. Progenitor observations were presented in Crockett et al. (2008) but the conclusions about the nature of the progenitor star were not clear. Photometric and spectroscopic data for SN 2008ax were taken from Pastorello et al. (2008), Roming et al. (2009), Tsvetkov et al. (2009), Taubenberger et al. (2011) and Chornock et al. (2011, hereafter C11).

The distance to the host galaxy NGC 4490 is not very well known. We have found only three measurements in the literature (Tully 1988; Terry et al. 2002; Theureau et al. 2007). Taking the median and standard deviation of these and the Virgo, Great Attractor and Shapley corrected kinematic distance as given by NED we get $9.38 \pm 0.85 \mathrm{Mpc}$ which we will adopt. The extinction within the Milky Way as given by the S98 extinction maps recalibrated by SF11 is $E(B-V)_{\mathrm{MW}}=0.02 \mathrm{mag}$. High resolution spectroscopy of the Na I D and K I $7699 \AA$ lines were presented in C11. The host galaxy NGC 4490 is a quite irregular galaxy so it is not clear if relations calibrated to the Milky Way are applicable. However, as we have no alternative, we will use the MZ97 relations to estimate the extinction within NGC 4490. The C11 spectra show blended multiple components of the $\mathrm{Na}_{\mathrm{I}} \mathrm{D}_{2}$ line most of which are clearly saturated. We measure the total equivalent width to $1.0 \AA$ which using the linear (unsaturated) part of the MZ97 relation corresponds to a lower limit of $E(B-V)_{\mathrm{H}}>0.25 \mathrm{mag}$. As the $\mathrm{Na}_{\mathrm{I}} \mathrm{D}_{2}$ lines are saturated we cannot use these to derive a useful upper limit. C11 measures the total equivalent width of the K I $7699 \AA$ line components to $0.142 \AA$ which using the corresponding MZ97 relation gives $E(B-V)_{\mathrm{H}}=0.54 \mathrm{mag}$. Adding the extinction within the Milky way and adopting the lower limit from the MZ97 Na I $\mathrm{D}_{2}$ relation and the extinction corresponding to the bluest SN colours allowed for a blackbody (Sect. 5.1) as the lower and upper error limits we then get $E(B-V)_{\mathrm{T}}=0.56_{-0.29}^{+0.14} \mathrm{mag}$.

\subsection{Comparison}

The left panel of Fig. 15 shows the pseudo-bolometric $U$ to $K$ (3000-24 $000 \AA$ ) lightcurves of SNe 2011dh, 1993J and 2008ax as calculated with the photometric method (Sect. 3.3). The absolute magnitudes have been calculated using cubic spline fits as described in Sect. 2.2.3 and extrapolated assuming constant colour. Except for the first few days the shape is similar and they all show the characteristics common to Type I and IIb SNe lightcurves (Sect. 5.2). As shown in B12 the differences during the first few days could be explained by differences in the radius and mass of the hydrogen envelope. Given the adopted distances and extinctions SN 2011dh is fainter than SN 1993J which, in turn, is fainter than SN 2008ax. The peak luminosity occurs at similar times but the peak-to-tail luminosity ratio for SN 2011dh is smaller than for SN 1993J which, in turn, is smaller than for SN 2008ax.

The left panel of Fig. 16 shows the colour evolution for the three SNe. The absolute magnitudes have been calculated using cubic spline fits as described in Sect. 2.2.3. As for the lightcurves, the shape is quite different for the first few days which could again be explained by differences in the radius and mass of the hydrogen envelope. The shape of the subsequent evolution is quite similar with a blueward trend in the $V-I$ and $V-K$ colours (corresponding to increasing temperature) during the rise to peak luminosity and then a redward trend in all colours (corresponding to decreasing temperature) to a colour maximum at 40-50 days and a subsequent slow blueward trend. Given the adopted extinction, SN 2011dh is redder than SN 1993J which, in turn, is redder than SN 2008ax.

In Figs. 17 and 18 we show closeups of the spectral evolution centred on the $\mathrm{H} \alpha$ and the $\mathrm{He}$ I $10830 \AA$ A lines. The minimum 

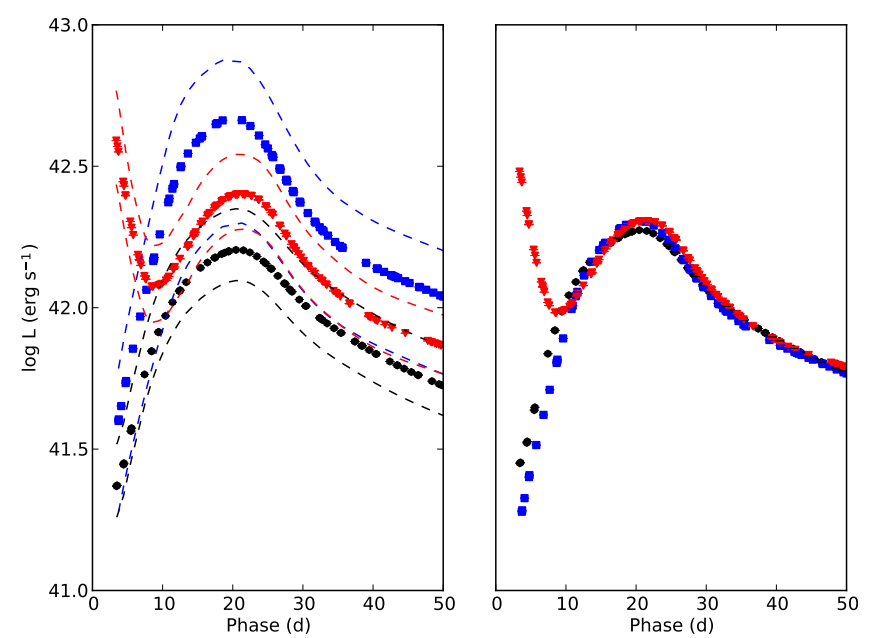

Fig. 15. Pseudo-bolometric $U$ to $K$ lightcurve for SN 2011dh (black circles) as compared to SNe 1993J (red triangles) and 2008ax (blue squares) for the adopted extinctions (left panel) and for a revised scenario where we have set $E(B-V)_{\mathrm{T}}$ to $0.14,0.09$ and 0.27 mag for SNe 2011dh, 1993J and 2008ax respectively (right panel). In the left panel we also show the systematic error arising from the distance and extinction (dashed lines).
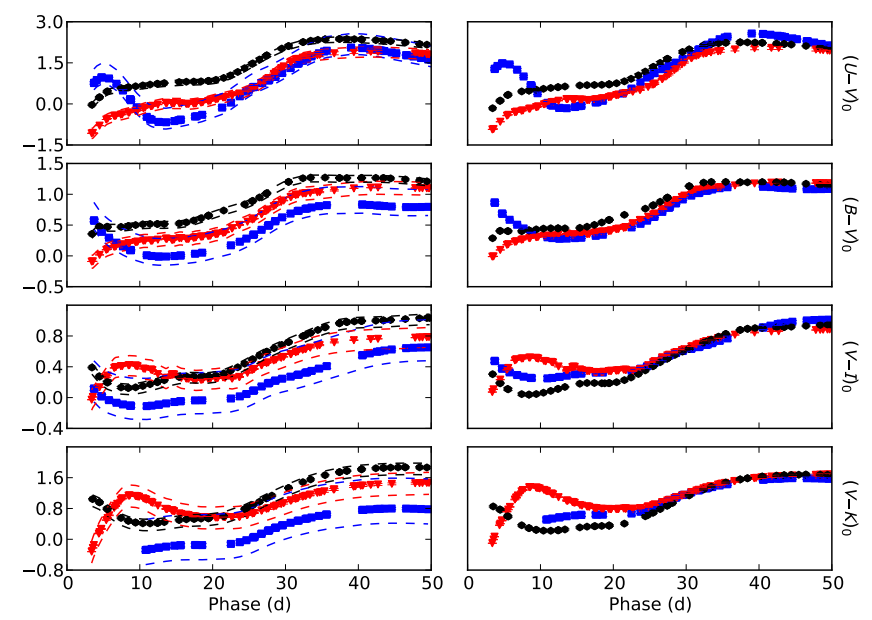

Fig. 16. Colour evolution of SN 2011dh (black circles) as compared to $\mathrm{SNe} 1993 \mathrm{~J}$ (red triangles) and 2008ax (blue squares) for the adopted extinctions (left panel) and for a revised scenario where we have set $E(B-V)_{\mathrm{T}}$ to $0.14,0.09$ and $0.27 \mathrm{mag}$ for SNe 2011dh, 1993J and 2008ax respectively (right panel). In the left panel we also show the systematic error arising from the extinction (dashed lines).

velocity for the $\mathrm{H} \alpha$ absorption minimum has been marked and occurs at $\sim 9000, \sim 11000$ and $\sim 13000 \mathrm{~km} \mathrm{~s}^{-1}$ for SNe 1993J, 2011dh and 2008ax respectively. As discussed in Sect. 3.4 this velocity likely corresponds to the interface between the helium core and the hydrogen envelope for SN $2011 \mathrm{dh}$. The $\mathrm{H} \alpha$ line disappears at $\sim 50$ days for SN 2008ax, at $\sim 80$ days for SN $2011 \mathrm{dh}$ and is still strong at 100 days for SN 1993J. Figure 19 shows the evolution of the absorption minimum for the Fe II $5169 \AA$, He 5876 and $6678 \AA$ and $\mathrm{H} \alpha$ lines measured as described in Sect. 3.4. Interpreting the Fe II $5169 \AA$ absorption minimum as the photosphere and the minimum velocity for the $\mathrm{H} \alpha$ absorption minimum as the interface between the helium core and the hydrogen envelope the photosphere reaches the helium core at $\lesssim 10$, $\sim 5$ and $\lesssim 10$ days for SNe 1993J, 2011dh and 2008ax respectively. The helium lines appear at $\sim 20, \sim 10$ and $\sim 5$ days for SNe 1993J, 2011dh and 2008ax respectively, at lower velocities

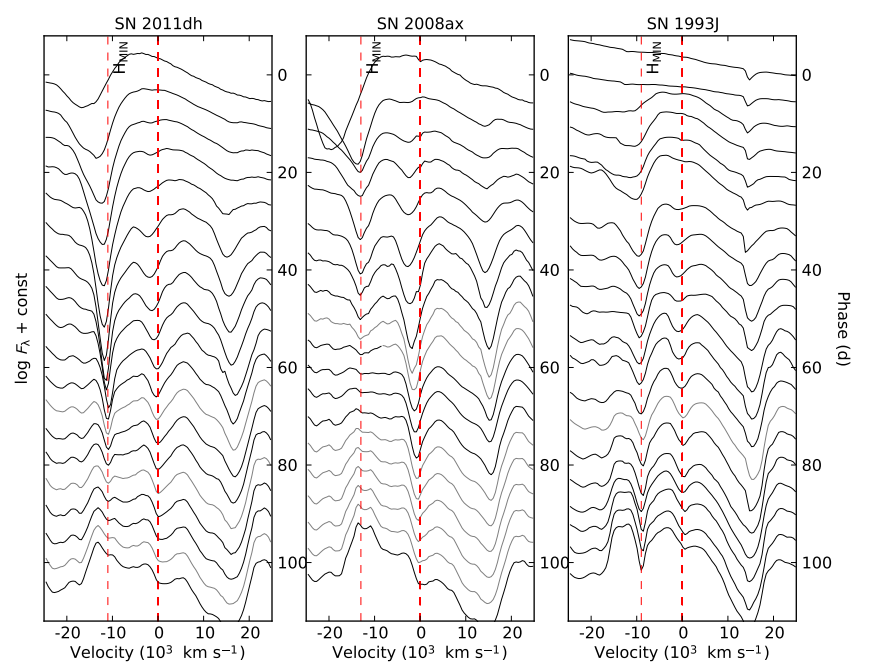

Fig. 17. The (interpolated) evolution of the $\mathrm{H} \alpha$ line for SN 2011 dh (left panel) as compared to SNe 2008ax (middle panel) and 1993J (right panel). All panels in this and the following figure show the minimum velocity for the $\mathrm{H} \alpha$ absorption minimum (marked $\mathrm{H}_{\text {MIN }}$ ) interpreted as the interface between the helium core and hydrogen envelope.

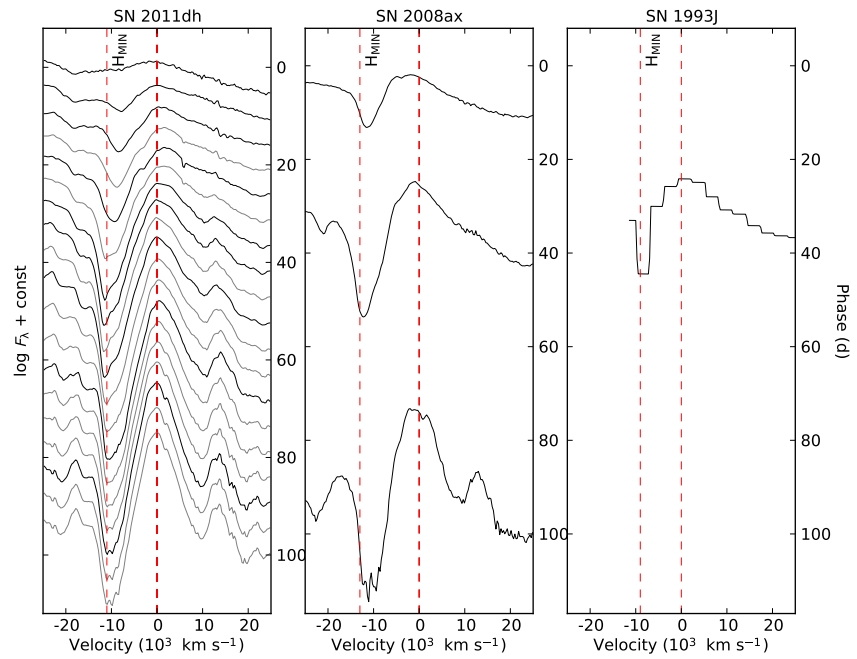

Fig. 18. The (interpolated) evolution of the He I $10830 \AA$ line for SN 2011dh (left panel) as compared to SNe 2008ax (middle panel) and 1993J (right panel). Given the sparse data available for SNe 1993J and 2008ax we show observed spectra for these SNe.

close to the region where we expect the continuum photosphere to be located. The initial evolution is different among the $\mathrm{SNe}$ but after $\sim 30$ days the helium lines have increased in strength, moved outward as compared to the photosphere and show a quite similar evolution for all three SNe. The evolution of the Fe II $5169 \AA$ Aine is very similar for SNe 1993J and 2011dh but a bit different for SN 2008ax. In general, lines originating closer to the photosphere seem to have similar velocities for the three $\mathrm{SNe}$ whereas lines originating further out in the ejecta seem to have progressively higher velocities for SNe 1993J, 2011dh and 2008ax respectively.

The differences in peak and tail luminosities suggest differences in the mass of ejected ${ }^{56} \mathrm{Ni}$ (Sect. 5.2). The differences in peak-to-tail luminosity ratios suggest differences in the ejecta mass, explosion energy and/or distribution of ${ }^{56} \mathrm{Ni}$ (Sect. 5.2). However, as seen in the left panels of Figs. 15 and 16 the systematic errors in the luminosity and colour arising from the distance 


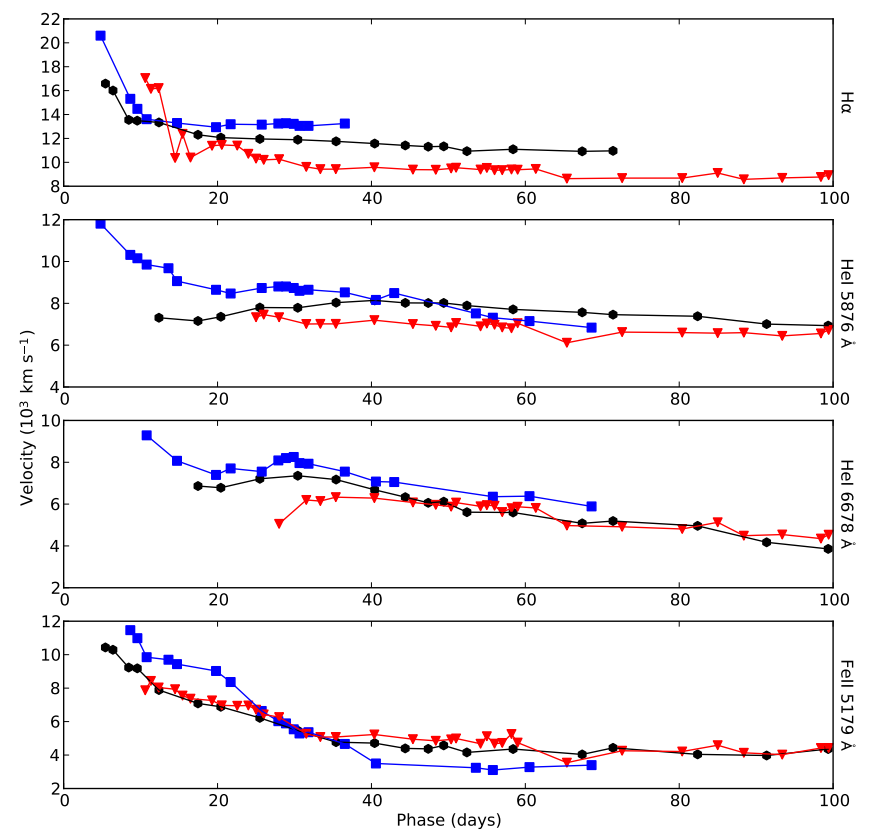

Fig. 19. Velocity evolution of the absorption minimum for the $\mathrm{H} \alpha$ (upper panel), He I $5876 \AA$ (upper middle panel), He I 6678 A (lower middle panel) and $\mathrm{Fe}$ II 5169 A (lower panel) lines for SNe 2011dh (black circles), 2008ax (blue squares) and 1993J (red triangles) measured as described in Sect. 3.4.

and extinction is large so similarity among the SNe cannot be excluded. M13 find both the luminosities and the colours to be similar, mainly due to differences in the adopted distances and extinctions. The similar velocities of lines originating closer to the photosphere and the times at which peak luminosity occurs, both of which are independent of the distance and extinction, suggests similar ejecta masses and explosion energies (Sect. 5.2). Although the differences in the bolometric lightcurves could possibly be explained by differences in the mass and distribution of ejected ${ }^{56} \mathrm{Ni}$ this is not fully satisfactory as the mass of ejected ${ }^{56} \mathrm{Ni}$ is known from observations to be correlated with initial mass and expansion velocity (Fraser et al. 2011; Maguire et al. 2012). In all the observed characteristic of the $\mathrm{SNe}$ does not seem entirely consistent and we have to consider the possibility that the adopted distances and extinctions are in error.

Interestingly enough, it is possible to revise the extinctions alone, within the adopted error bars, in such a way that it brings the colour evolution, the bolometric luminosities and the peakto-tail luminosity ratios in good agreement. This is shown in the right panels of Figs. 15 and 16 where we have set $E(B-V)_{\mathrm{T}}$ to $0.14,0.09$ and $0.27 \mathrm{mag}$ for SNe 2011dh, 1993J and 2008ax respectively. Intrinsic differences among the $\mathrm{SNe}$ can not be excluded and the arguments used are only suggestive so we can not make a definite conclusion. It is clear, however, that a scenario where all three SNe have similar ejecta masses, explosion energies and ejected masses of ${ }^{56} \mathrm{Ni}$ is possible. As shown in B12 the differences in the early evolution and the velocities of lines originating further out in the ejecta could be explained by differences in the mass and radius of the hydrogen envelope. The progressively higher minimum velocities for the $\mathrm{H} \alpha$ absorption minimum, if interpreted as the interface between the helium core and the hydrogen envelope, would naively suggest progressively lower masses of this envelope for SNe 1993J, $2011 \mathrm{dh}$ and 2008ax respectively. Such a conclusion is supported by the early photometric evolution, the strength and persistence of the $\mathrm{H} \alpha$ line, the hydrodynamical modelling of SNe 1993J and 2011dh in B12, the spectral modelling of SN 2011dh in this paper and by A11 and the spectral modelling of SN 2008ax by Maurer et al. (2010). Marion et al. (2013) reach a similar conclusion based on the progressively later times at which the helium lines appears although we do not find their physical argument convincing (Sect. 3.4).

\section{Discussion}

In Sect. 5.1 we revisit the issue of extinction and discuss constraints from the SN itself and the comparisons to SNe 1993J and 2008ax made in Sect. 4.3. In Sect. 5.2 we discuss the physics of Type IIb lightcurves as understood from approximate models, in particular in relation to the hydrodynamical modelling made in B12. In Sect. 5.3 we discuss the sensitivity of the SN and progenitor parameters derived in B12 to errors in the distance, extinction and photospheric velocity and also revise these parameters to agree with the distance and extinction adopted in this paper. In Sect. 5.4 we discuss the results on the disappearance of the progenitor star and what consequences this have for the results in M11 and B12 and our understanding of this star and Type IIb progenitors in general. Finally, in Sect. 5.5, we discuss the excess in the Spitzer $4.5 \mu \mathrm{m}$ band and possible explanations.

\subsection{Extinction revisited}

In Sect. 1.3 we discussed different estimates of the extinction for SN 2011dh. Most estimates suggested a low extinction and we adopted $E(B-V)=0.07_{-0.04}^{+0.07} \mathrm{mag}$ as estimated from the equivalent widths of the Na I D lines. The near simultaneous $V$ and $R$ band observations from day 1 presented in A11 and T12 corresponds to an intrinsic $V-R$ colour of about -0.2 mag for the adopted extinction. The bluest $V-R$ colour allowed for a blackbody, which can be calculated from the Rayleigh-Jeans law, is -0.16 mag so this suggests a very high temperature. In higher extinction scenarios this colour would be even bluer and, even taking measurement and calibration errors into account, in conflict with the bluest $V-R$ colour allowed for a blackbody. Figure 5 shows the evolution of the blackbody temperature for two higher extinction scenarios where we have increased $E(B-V)_{\mathrm{T}}$ in $\sim 0.1$ steps to 0.2 and $0.3 \mathrm{mag}$. As seen the blackbody temperature would become quite high between 10 and 20 days and we would expect lines from low ionization potential ions such as $\mathrm{Ca}$ II and Fe II to be quite sensitive to this. As shown in Fig. 11, the SN atmosphere code described in Sect. 3.4 can neither reproduce the Ca II 8498/8542/8662 A lines, nor the Fe II lines, between 10 and 20 days for these higher extinction scenarios. Even though NLTE effects may change the ion fractions, this again suggests a low extinction scenario for SN 2011dh. Comparisons to SNe 2008ax and 1993J provides another source of information. As discussed in Sect. 4 an assumption of similarity in luminosity and colour among the $\mathrm{SNe}$ requires a revision of the extinctions adopted in this paper and suggest a revision of the extinction for SN 2011dh towards the upper error bar. However, as pointed out, intrinsic differences among the $\mathrm{SNe}$ cannot be excluded and as such a revision would be within our error bars we do not find this argument sufficient to revise our adopted value $E(B-V)=0.07_{-0.04}^{+0.07} \mathrm{mag}$.

\subsection{Physics of Type IIb SNe lightcurves}

The bolometric lightcurves of SN 2011dh and other Type IIb $\mathrm{SNe}$ can be divided in two distinct phases depending on the 
energy source powering the lightcurve. The first phase is powered by the thermal energy deposited in the ejecta by the explosion. The second phase is powered by the energy deposited in the ejecta by the $\gamma$-rays emitted in the radioactive decay chain of ${ }^{56} \mathrm{Ni}$. In B12 we used the ${ }^{56} \mathrm{Ni}$ powered phase to estimate the ejecta mass, explosion energy and ejected mass of ${ }^{56} \mathrm{Ni}$ whereas the explosion energy powered phase was used to estimate the radius of the progenitor star.

The explosion energy powered phase ends at $\sim 3$ days when our observations begin but $V R$ and $g$ band data have been published in A11 and T12. These data are insufficient to construct a bolometric lightcurve but it is clear that this phase corresponds to a strong decline of the bolometric luminosity. In the B12 modelling most of the thermal energy is transformed to kinetic energy before shock breakout due to expansion. The thermal energy present in the envelope at shock breakout is only a small fraction of the explosion energy and it is the cooling of this, mainly by expansion and partly by radiative diffusion, that gives rise to the strong decline of the bolometric luminosity. The shape and extent of the bolometric lightcurve in the explosion energy powered phase depends on the mass, radius, density profile and composition of the envelope and, as discussed in B12, requires detailed hydrodynamical modelling.

The subsequent ${ }^{56} \mathrm{Ni}$ powered phase is well covered by our data and the bolometric lightcurve (Fig. 8) shows the characteristics common to all Type I and IIb SNe; a rise to peak luminosity followed by a decline phase and a subsequent tail phase with a roughly linear decline rate. These characteristics can be qualitatively understood by approximate models such as the ones by Arnett (1982) or Imshennik \& Popov (1992). The rising phase is caused by radiative diffusion of the energy deposited in the ejecta by the $\gamma$-rays. The radioactive heating decreases with time and so does the diffusion time because the ejecta are expanding. As shown by Arnett (1982) the luminosity peak is reached when the radioactive heating equals the cooling by radiative diffusion. During the subsequent decline phase the diffusion time continues to decrease until the $\mathrm{SN}$ reaches the tail phase where the diffusion time is negligible and the luminosity equals the radioactive heating (instant diffusion). The shape of the tail is not exactly linear but is modulated by a term determined by the decreasing optical depth for $\gamma$-rays as the ejecta continue to expand.

From approximate models the qualitative dependence of the bolometric lightcurve in the ${ }^{56} \mathrm{Ni}$ powered phase on basic parameters as the explosion energy, ejecta mass and mass of ejected ${ }^{56} \mathrm{Ni}$ can be understood. Increasing the explosion energy will increase the expansion velocities which will decrease the diffusion time for thermal radiation and the optical depth for $\gamma$-rays. Increasing the ejecta mass will have the opposite effect but, as the optical depth $\tau \propto\left(M^{2} / E\right)$ and the diffusion time $t_{\mathrm{d}} \propto\left(M^{3} / E\right)^{1 / 4}$ (Arnett 1982), the bolometric lightcurve depends stronger on the ejecta mass than on the explosion energy. Either an increase of the explosion energy or a decrease of the ejecta mass will result in an earlier and more luminous peak of the bolometric lightcurve whereas the tail luminosity will be decreased. Increasing the mass of ${ }^{56} \mathrm{Ni}$ will increase the radioactive heating and thus result in an overall increase of the luminosity and in fact corresponds to a pure scaling in the approximate models. The distribution of ${ }^{56} \mathrm{Ni}$ also affects the lightcurve and if the ${ }^{56} \mathrm{Ni}$ is distributed further out in the ejecta the lightcurve will rise faster to the peak because of the decreased diffusion time for thermal radiation and have a lower luminosity on the tail because of the decreased optical depth for $\gamma$-rays. As shown in Figs. 2 and 4-6 in B12 all these qualitative dependencies are well followed by the hydrodynamical models.

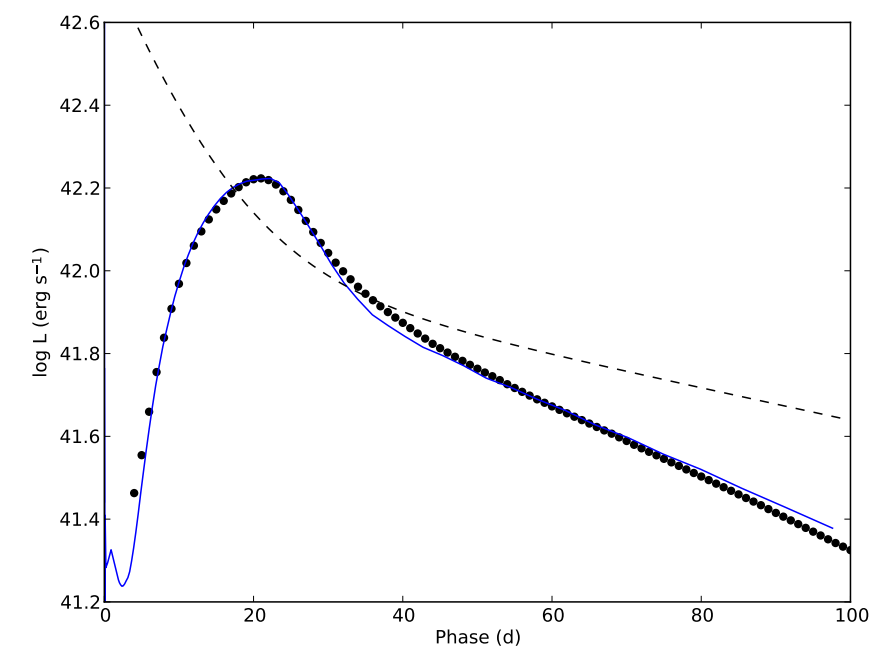

Fig. 20. Revised B12 He4 model bolometric lightcurve with the ${ }^{56} \mathrm{Ni}$ mass increased to $0.075 M_{\odot}$ (blue solid line) compared to the pseudo-bolometric UV to MIR lightcurve for SN 2011dh calculated with the spectroscopic method (black dots). For comparison we also show the total $\gamma$-ray luminosity corresponding to this amount of ${ }^{56} \mathrm{Ni}$ (black dashed line).

If the optical depth for $\gamma$-rays in the tail phase is high the shape of the bolometric lightcurve in the ${ }^{56} \mathrm{Ni}$ powered phase depends exclusively on the diffusion time for thermal radiation, which determines the quantity $\left(M^{3} / E\right)$, and the ejecta mass and explosion energy become degenerate. In this case knowledge of the expansion velocity, which determines the quantity $(M / E)$, is needed to determine the $\mathrm{SN}$ parameters. However, as seen in Fig. 20, the optical depth for $\gamma$-rays becomes $\lesssim 1$ at $\sim 40$ days for SN 2011dh. The bolometric lightcurve in the tail phase then depends on the optical depth for $\gamma$-rays, which determines the quantity $\left(M^{2} / E\right)$, and provides the constraint needed to break the degeneracy. However, as the bolometric lightcurve also depends on the distribution of ${ }^{56} \mathrm{Ni}$ the problem is not necessarily well-conditioned. In our experience, knowledge of the expansion velocity, which corresponds to the fitting of photospheric velocities in B12, is needed to robustly determine the SN parameters.

\subsection{Error sensitivity and revisions of the B12 modelling}

What was not discussed in B12 was the sensitivity of the results to errors in the adopted distance and extinction. A change in the distance corresponds to a scaling of the bolometric lightcurve whereas a change in the extinction is more complicated as the change in luminosity also depends on the colour. However, as seen in Fig. 8, the change in luminosity for SN 2011dh due to the combined errors in distance and extinction does not differ significantly from a scaling. As the adopted distance and extinction have been revised as compared to B12 we also need to investigate the effect of this change on the derived quantities.

In the ${ }^{56} \mathrm{Ni}$ powered phase, according to approximate models, the luminosity is proportional to the mass of ejected ${ }^{56} \mathrm{Ni}$ (Sect. 5.2). Therefore, ignoring possible degeneracy among the parameters, we expect the derived ejecta mass and explosion energy to be insensitive to the errors in the distance and extinction and the error in the derived mass of ejected ${ }^{56} \mathrm{Ni}$ to be similar to the error in the luminosity. We have re-run the $\mathrm{He} 4$ model with the ${ }^{56} \mathrm{Ni}$ mass increased to $0.075 M_{\odot}$ to account for the revisions in the adopted distance and extinction. The model bolometric lightcurve is shown in Fig. 20 and well reproduces the bolometric lightcurve presented in this paper. Estimating the errors 
arising from the distance and extinction as described the revised mass of ejected ${ }^{56} \mathrm{Ni}$ becomes $0.05-0.10 M_{\odot}$ whereas the ejecta mass and explosion energy remains the same as in B12, $1.8-2.5 M_{\odot}$ and $0.6-1.0 \times 10^{51} \mathrm{erg}$ respectively.

In the explosion energy powered phase, according to approximate models, the luminosity is proportional to the radius. However, the lightcurve in this phase is not well described by approximate models and detailed hydrodynamical modelling is needed (Sect. 5.2). As discussed in B12 the sensitivity of the estimated radius to changes in the luminosity is modest. Examining the range of envelope models consistent with the A11 $g$ band observations, the distance and extinction adopted in this paper and the systematic error arising from these we find a revised progenitor radius of $200-300 R_{\odot}$.

As discussed in Sect. 3.4 the region where the Fe II $5169 \AA$ line is formed rather provides an upper limit for the location of the photosphere and the photospheric velocities used in the hydrodynamical modelling might therefore be overestimated. The dependence of the derived quantities on the photospheric velocity is complicated and a full scan of the model parameter space is probably needed to make a quantitative estimate. This is a potential problem and further work is needed to well constrain the photospheric velocities.

\subsection{Disappearance of the proposed progenitor star}

In Ergon et al. (2013) we presented $B, V$ and $r$ observations of the SN site obtained on Jan. 202013 ( $V$ and $r$ ) and Mar. 192013 $(B), 601$ and 659 days past explosion respectively. An additional set of $B, V$ and $r$ band observations was obtained on Apr. 14 2013, May 152013 and Jun. 1 2013, 685, 715 and 732 days past explosion. In Appendix B we give the details on these observations and the photometric measurements and calibration. Subtraction of pre-explosion images shows that the flux from the yellow supergiant proposed as the progenitor by M11 have been reduced with at least $74 \pm 9,73 \pm 4$ and $77 \pm 4$ percent in the $B, V$ and $r$ bands respectively. The HST observations obtained on Mar 22013 and presented by D13 corresponds to a reduction of the flux of $71 \pm 1$ and $70 \pm 1$ in the $F 555 W$ and $F 814 W$ bands respectively. Szczygieł et al. (2012) find the progenitor to be variable at the five percent level so variability of the star is unlikely to explain the flux reduction. We find a $0.57,0.76$ and $0.64 \mathrm{mag}$ decline corresponding to a decline rate of $0.0073,0.0090$ and $0.0053 \mathrm{mag} \mathrm{day}^{-1}$ in the $B, V$ and $r$ bands respectively between the first and the second set of observations which is consistent with the remaining flux being emitted by the SN. As can be derived from the approximate models discussed in Sect. 5.2, in the limit of low optical depth for the $\gamma$-rays and if all positrons are trapped, the decline rate is $\left(1+\left(1-f_{\mathrm{e}^{+}}(t)\right) 223 / t\right) 0.0098 \mathrm{mag} \mathrm{day}^{-1}$, where $f_{\mathrm{e}+}(t)$ is the fractional positron contribution to the luminosity and $t$ is given in days. This gives an expected decline rate of of $0.0098-0.0132 \mathrm{mag}$ at 650 days, in rough agreement with the observed decline rates if the contribution from positrons is assumed to dominate. Given all this, although we can not exclude a minor contribution to the pre-explosion flux from other sources, we find that the yellow supergiant has disappeared. The only reasonable explanation is that the star was the progenitor of SN 2011dh as originally proposed in M11 which is also the conclusion reached by D13.

The disappearance of the yellow supergiant confirms the results in B12 in which we showed that an extended progenitor with the observed properties of the yellow supergiant could well reproduce the early optical evolution. This shows that the duration of the initial cooling phase for a $\mathrm{SN}$ with an extended progenitor can be significantly shorter than commonly thought and that approximate models as the one by Rabinak \& Waxman (2011) used in A11 does not necessarily apply. It also indicates that the proposed division of Type IIb SNe progenitors in compact and extended ones and the relation between the speed of the shock and the type of progenitor (Chevalier \& Soderberg 2010) needs to be revised. It is interesting to note that the two progenitors of Type IIb SNe (1993J and 2011dh) whose nature have been revealed were in both cases extended supergiants. The disappearance of the yellow supergiant in M51 was a major step in achieving one of our main goals, to determine the initial mass of the progenitor star. This mass has now been estimated to $\sim 13 M_{\odot}$ by two different methods, the hydrodynamical modelling in B12 and the progenitor analysis in M11, respectively. Both methods use results from stellar evolutionary modelling to relate the He core mass and the progenitor luminosity respectively to the initial mass but are otherwise independent. We note that, contrary to most other types, the initial mass of Type IIb $\mathrm{SNe}$ progenitors might be derived from hydrodynamical modelling without any assumptions of uncertain mass-loss rates as the star is essentially a bare He core (although with a thin and extended envelope).

\section{5. $4.5 \mu \mathrm{m}$ excess}

As mentioned in Sects. 3.1 and 3.3 there is a flux excess in the Spitzer $4.5 \mu \mathrm{m}$ band as compared to the 2MASS JHK and Spitzer $3.6 \mu \mathrm{m}$ bands developing during the first 100 days. This is most clearly seen in Fig. 10. Whereas other bands redward of $V$ are well approximated by the blackbody fits the flux at $4.5 \mu \mathrm{m}$ is a factor of $\sim 5$ in excess at 100 days. Warm dust or $\mathrm{CO}$ fundamental band emission are two possible explanations. For day 50-100 the excess (relative the blackbody fits discussed in Sect. 3.2) is well fitted by a blackbody with $T \simeq 400 \mathrm{~K}$, $R \simeq 5 \times 10^{16} \mathrm{~cm}$ and $L \simeq 6 \times 10^{40} \mathrm{erg} \mathrm{s}^{-1}$. Helou et al. (2013) find a blackbody luminosity declining from $\sim 7 \times 10^{40}$ to $\sim 3 \times 10^{40} \mathrm{erg} \mathrm{s}^{-1}$ and cooling from $\sim 1600$ to $\sim 600 \mathrm{~K}$ between $\sim 20$ and $\sim 90$ days by fitting the MIR bands alone. Using a simple model for heated CSM dust they also find a thermal dust echo to be consistent with the observed MIR fluxes. Given the lack of MIR spectra we can neither confirm nor excluded $\mathrm{CO}$ fundamental band emission as the explanation of the excess. There is a possible excess (as compared to the continuum) developing near the location of the first overtone band at $\sim 23000 \AA$ though. NIR spectra from later epochs may help to resolve this issue as we expect first overtone emission to grow stronger as compared to the continuum.

\section{Conclusions}

We present extensive photometric and spectroscopic optical and NIR observations of SN 2011dh obtained during the first 100 days. The calibration of the photometry is discussed in some detail and we find it to be accurate to the five percent level in all bands, except for the early (0-40 days) NOT $U$ band observations. Using our observations as well as Swift UV and Spitzer MIR observations we calculate the bolometric UV to MIR lightcurve using both photometric and spectroscopic data. This bolometric lightcurve together with the photospheric velocity as estimated from the absorption minimum of the Fe II $5169 \AA$ line provides the observational basis for the hydrodynamical modelling done in B12.

We adopt a distance of $7.8_{-0.9}^{+1.1} \mathrm{Mpc}$ based on all estimates in the literature and find an extinction of $E(B-V)_{\mathrm{T}}=0.07_{-0.04}^{+0.07} \mathrm{mag}$ 
to be consistent with estimates and constraints presented in the literature and in this paper. The sensitivity of the results in B12 to these uncertainties is discussed and we find that only the derived mass of ejected ${ }^{56} \mathrm{Ni}$ and radius is likely to be affected. We also revise the modelling made in B12 to agree with the values of the distance and extinction adopted in this paper and find that only the derived mass of ejected ${ }^{56} \mathrm{Ni}$ and radius needs to be revised. The uncertainty in the photospheric velocity as estimated from the absorption minimum of the Fe II $5169 \AA$ line is discussed and we find that we can not constrain this velocity very well. This is a potential problem as we are unable to quantify the sensitivity of the results in B12 to this uncertainty.

We present and discuss pre- and post-explosion observations which show that the yellow supergiant coincident with SN 2011dh has disappeared and indeed was the progenitor as proposed in M11. Furthermore, the results from the progenitor analysis in M11 are consistent with those from the hydrodynamical modelling in B12. Given the revisions in this paper, we find that an almost bare helium core with a mass of 3.3-4.0 $M_{\odot}$ surrounded by a thin hydrogen rich envelope extending to $200-300 R_{\odot}$ exploded with an energy of $0.6-1.0 \times$ $10^{51} \mathrm{erg}$ ejecting a mass of $1.8-2.5 M_{\odot}$ of which $0.05-0.10 M_{\odot}$ consisted of synthesised ${ }^{56} \mathrm{Ni}$.

The absorption minimum of the hydrogen lines is never seen below $\sim 11000 \mathrm{~km} \mathrm{~s}^{-1}$ but approaches this value when the lines get weaker. Spectral modelling of the hydrogen lines using the B12 He4R270 ejecta model well reproduces this behaviour and the minimum velocity of the absorption minima coincides with the model interface between the helium core and the hydrogen rich envelope. The good agreement between the modelled and observed minimum velocities gives support to the B12 He4R270 ejecta model and we find it most likely that the observed minimum velocity of $\sim 11000 \mathrm{~km} \mathrm{~s}^{-1}$ corresponds to the interface between the helium core and the hydrogen rich envelope. We note that the minimum velocity of the $\mathrm{H} \alpha$ absorption minimum for SNe 1993J, 2011dh and 2008ax is $\sim 9000, \sim 11000$ and $\sim 13000 \mathrm{~km} \mathrm{~s}^{-1}$ respectively which suggest that the interface between the helium core and the hydrogen rich envelope is located near these progressively higher velocities. By varying the fraction of hydrogen in the envelope we find a hydrogen mass of $0.01-0.04 M_{\odot}$ to be consistent with the observed evolution of the hydrogen lines. This is in reasonable agreement with the $0.02 M_{\odot}$ in the original model and the $0.024 M_{\odot}$ estimated by A11 using spectral modelling similar to the one in this paper. We estimate that the photosphere reaches the interface between the helium core and the hydrogen rich envelope at 5-7 days. The helium lines appear between $\sim 10$ days (He I 10830 and $5876 \AA$ ) and $\sim 15$ days (He I 6678, 7065 and $20581 \AA$ ), close to the region where we expect the photosphere to be located and then move outward in velocity until $\sim 40$ days. This suggests that the early evolution of these lines is driven by increasing non-thermal excitation due to decreasing optical depth for the $\gamma$-rays.

The photometric and spectral characteristics of SNe 2011dh, 1993J and 2008ax are compared and we find the colours and luminosities to differ significantly for the distances and extinctions adopted in this paper. However, the errors arising from the distance and extinction are large and a revision of the extinctions, just within the error bars, would bring the colours and luminosities in good agreement. Although a definite conclusion can not be made it is clear that a scenario where all three SNe have similar ejecta masses, explosion energies and ejected masses of ${ }^{56} \mathrm{Ni}$ is possible. As shown in B12 the differences in the early evolution could be explained by differences in the mass and radius of the hydrogen envelope. Progressively higher velocities of the interface between the helium core and the hydrogen rich envelope, as proposed above, would naively correspond to progressively lower masses of this envelope for SNe 1993J, 2011dh and 2008ax respectively. Such a conclusion is supported by the early photometric evolution, the strength and persistence of the $\mathrm{H} \alpha$ line and hydrodynamical as well as spectral modelling of these $\mathrm{SNe}$.

We detect a flux excess in the $4.5 \mu \mathrm{m}$ Spitzer band as compared to the NIR and the $3.6 \mu \mathrm{m}$ Spitzer band. A thermal dust echo in the CSM as proposed by Helou et al. (2013) or CO fundamental band emission are possible explanations but further work using late time observations is needed to resolve this issue.

The high quality dataset presented in this paper provides an ideal base for further modelling of the SN. One of the most interesting issues which remains unsolved is the possible existence of a bluer and more compact companion star as predicted by the binary evolutionary modelling in Benvenuto et al. (2013). It is still not clear which of the single or binary star channels is dominating the production of Type IIb SNe. HST imaging, preferably in the UV, would have a good chance to detect such a companion.

Acknowledgements. This work is partially based on observations of the European supernova collaboration involved in the ESO-NTT and TNG large programmes led by Stefano Benetti. This work is partially based on observations made with the Nordic Optical Telescope, operated by the Nordic Optical Telescope Scientific Association at the Observatorio del Roque de los Muchachos, La Palma, Spain, of the Instituto de Astrofísica de Canarias. We acknowledge the exceptional support we got from the NOT staff throughout this campaign. This work is partially based on observations made with the Italian Telescopio Nazionale Galileo (TNG) operated by the Fundacion Galileo Galilei of the INAF (Istituto Nazionale di Astrofisica) at the Spanish Observatorio del Roque de los Muchachos of the Instituto de Astrofísica de Canarias; the $1.82 \mathrm{~m}$ Copernico and Schmidt 67/92 telescopes of INAF - Asiago Observatory; the $1.22 \mathrm{~m}$ Galileo telescope of Dipartimento di Fisica e Astronomia (Universita' di Padova); the LBT, which is an international collaboration among institutions in the United States, Italy, and Germany. LBT Corporation partners are The Ohio State University, and The Research Corporation, on behalf of the University of Notre Dame, University of Minnesota and University of Virginia; the University of Arizona on behalf of the Arizona university system; INAF, Italy. We are in debt with S. Ciroi, A. Siviero and L. Aramyan for help with the Galileo $1.22 \mathrm{~m}$ observations. This work is partially based on observation made with the William Herschel Telescope, operated on the island of La Palma by the Isaac Newton Group in the Spanish Observatorio del Roque de los Muchachos of the Instituto de Astrofísica de Canarias and the Liverpool Telescope, operated on the island of La Palma by Liverpool John Moores University in the Spanish Observatorio del Roque de los Muchachos of the Instituto de Astrofísica de Canarias with financial support from the UK Science and Technology Facilities Council. This work is partially based on observations made with the Carlos Sánchez Telescope operated on the island of Tenerife by the Instituto de Astrofísica de Canarias in the Spanish Observatorio del Teide, and the Joan Oró Telescope of the Montsec Astronomical Observatory, which is owned by the Generalitat de Catalunya and operated by the Institute for Space Studies of Catalonia (IEEC). L.T., A.P., S.B., E.C., and M.T. are partially supported by the PRIN-INAF 2011 with the project Transient Universe: from ESO Large to PESSTO. N.E.R. acknowledges financial support by the MICINN grant AYA08-1839/ESP, AYA201124704/ESP, and by the ESF EUROCORES Program EuroGENESIS (MINECO grants EUI2009-04170). S.T. acknowledges support by TRR 33 "The Dark Universe" of the German Research Foundation. J.S. and the OKC are supported by The Swedish Research Council. R.K. and M.T. gratefully acknowledges the allocation of Liverpool Telescope time under the programmes ITP10-04 and PL11A-03 on which this study is partially-based. F.B. acknowledges support from FONDECYT through grant 3120227 and by the Millennium Center for Supernova Science through grant P10-064-F (funded by "Programa Bicentenario de Ciencia y Tecnología de CONICYT" and "Programa Iniciativa Científica Milenio de MIDEPLAN"). Finally, we thank Vallery Stanishev for providing the NOT JC colour terms and the referee Jozsef Vinko for his useful suggestions.

\section{References}

Abbott, D. C., \& Lucy, L. B. 1985, ApJ, 288, 679 Aihara, H., Allende Prieto, C., An, D., et al. 2011, ApJS, 193, 29 
Arcavi, I., Gal-Yam, A., Yaron, O., et al. 2011, ApJ, 742, L18

Arnett, W. D. 1982, ApJ, 253, 785

Baron, E., Hauschildt, P. H., Branch, D., et al. 1995, ApJ, 441, 170

Baron, E., Hauschildt, P. H., Branch, D., Kirshner, R. P., \& Filippenko, A. V. 1996, MNRAS, 279, 799

Baron, E., Branch, D., \& Hauschildt, P. H. 2007, ApJ, 662, 1148

Benvenuto, O. G., Bersten, M. C., \& Nomoto, K. 2013, ApJ, 762, 74

Bersten, M. C., Benvenuto, O. G., Nomoto, K., et al. 2012, ApJ, 757, 31

Bessell, M., \& Murphy, S. 2012, PASP, 124, 140

Bietenholz, M. F., Brunthaler, A., Soderberg, A. M., et al. 2012, ApJ, 751, 125

Blinnikov, S. I., Eastman, R., Bartunov, O. S., Popolitov, V. A., \& Woosley, S. E. 1998, ApJ, 496, 454

Bowen, D. V., Roth, K. C., Blades, J. C., \& Meyer, D. M. 1994, ApJ, 420, L71

Branch, D., Baron, E. A., \& Jeffery, D. J. 2003, Supernovae and Gamma-Ray Bursters, Lect. Notes Phys., 598, 47

Campana, S., \& Immler, S. 2012, MNRAS, 427, L70

Cardelli, J. A., Clayton, G. C., \& Mathis, J. S. 1989, ApJ, 345, 245

Chevalier, R. A., \& Soderberg, A. M. 2010, ApJ, 711, L40

Chornock, R., Filippenko, A. V., Li, W., et al. 2011, ApJ, 739, 41

Ciardullo, R., Feldmeier, J. J., Jacoby, G. H., et al. 2002, ApJ, 577, 31

Cohen, M., Wheaton, W. A., \& Megeath, S. T. 2003, AJ, 126, 1090

Crockett, R. M., Eldridge, J. J., Smartt, S. J., et al. 2008, MNRAS, 391, L5

Dessart, L., \& Hillier, D. J. 2005, A\&A, 439, 671

Dessart, L., Blondin, S., Brown, P. J., et al. 2008, ApJ, 675, 644

Doi, M., Tanaka, M., Fukugita, M., et al. 2010, AJ, 139, 1628

Ergon, M., Sollerman, J., Pursimo, T., et al. 2013, ATel, 4912, 1

Feldmeier, J. J., Ciardullo, R., \& Jacoby, G. H. 1997, ApJ, 479, 231

Fraser, M., Ergon, M., Eldridge, J. J., et al. 2011, MNRAS, 417, 1417

Georgiev, T. B., Getov, R. G., Zamanova, V. I., \& Ivanov, G. R. 1990, Pis ma Astronomicheskii Zhurnal, 16, 979

Griga, T., Marulla, A., Grenier, A., et al. 2011, Central Bureau Electronic Telegrams, 2736, 1

Helou, G., Kasliwal, M. M., Ofek, E. O., et al. 2013, ApJ, 778, L19

Hora, J. L., Carey, S., Surace, J., et al. 2008, PASP, 120, 1233

Horesh, A., Stockdale, C., Fox, D. B., et al. 2013, MNRAS, 436, 1258

Imshennik, V. S., \& Popov, D. V. 1992, AZh, 69, 497

Iwamoto, K., Nomoto, K., Hoflich, P., et al. 1994, ApJ, 437, L115

Jerkstrand, A., Fransson, C., \& Kozma, C. 2011, A\&A, 530, A45

Jerkstrand, A., Fransson, C., Maguire, K., et al. 2012, A\&A, 546, A28

Krauss, M. I., Soderberg, A. M., Chomiuk, L., et al. 2012, ApJ, 750, L40

Landolt, A. U. 1983, AJ, 88, 439

Landolt, A. U. 1992, AJ, 104, 340

Lewis, J. R., Walton, N. A., Meikle, W. P. S., et al. 1994, MNRAS, 266, L27

Lucy, L. B. 1991, ApJ, 383, 308

Maeda, K. 2012, ApJ, 758, 81

Maguire, K., Jerkstrand, A., Smartt, S. J., et al. 2012, MNRAS, 420, 3451

Marion, G. H., Vinko, J., Kirshner, R. P., et al. 2013, ApJ, submitted [arXiv: 1303.5482]

Martí-Vidal, I., Tudose, V., Paragi, Z., et al. 2011, A\&A, 535, L10

Matthews, K., Neugebauer, G., Armus, L., \& Soifer, B. T. 2002, AJ, 123, 753

Maund, J. R., Smartt, S. J., Kudritzki, R. P., Podsiadlowski, P., \& Gilmore, G. F. 2004, Nature, 427, 129

Maund, J. R., Fraser, M., Ergon, M., et al. 2011, ApJ, 739, L37

Maurer, I., Mazzali, P. A., Taubenberger, S., \& Hachinger, S. 2010, MNRAS, 409,1441

Mazzali, P. A., \& Lucy, L. B. 1993, A\&A, 279, 447

Munari, U., \& Zwitter, T. 1997, A\&A, 318, 269
Murphy, J. W., Jennings, Z. G., Williams, B., Dalcanton, J. J., \& Dolphin, A. E. 2011, ApJ, 742, L4

Oke, J. B., \& Gunn, J. E. 1983, ApJ, 266, 713

Pastorello, A., Kasliwal, M. M., Crockett, R. M., et al. 2008, MNRAS, 389, 955

Pastorello, A., Valenti, S., Zampieri, L., et al. 2009, MNRAS, 394, 2266

Podsiadlowski, P., Hsu, J. J. L., Joss, P. C., \& Ross, R. R. 1993, Nature, 364, 509

Poole, T. S., Breeveld, A. A., Page, M. J., et al. 2008, MNRAS, 383, 627

Poznanski, D., Butler, N., Filippenko, A. V., et al. 2009, ApJ, 694, 1067

Poznanski, D., Ganeshalingam, M., Silverman, J. M., \& Filippenko, A. V. 2011, MNRAS, 415, L81

Poznanski, D., Prochaska, J. X., \& Bloom, J. S. 2012, MNRAS, 426, 1465

Rabinak, I., \& Waxman, E. 2011, ApJ, 728, 63

Richmond, M. W., Treffers, R. R., Filippenko, A. V., et al. 1994, AJ, 107, 1022

Richmond, M. W., Treffers, R. R., Filippenko, A. V., \& Paik, Y. 1996, AJ, 112, 732

Ritchey, A. M., \& Wallerstein, G. 2012, ApJ, 748, L11

Roming, P. W. A., Pritchard, T. A., Brown, P. J., et al. 2009, ApJ, 704, L118

Rosse, W. P. 1850, Phil. Trans. R. Soc., 499

Sahu, D. K., Anupama, G. C., \& Chakradhari, N. K. 2013, MNRAS, 433, 2

Sandage, A., \& Tammann, G. A. 1974, ApJ, 194, 559

Sasaki, M., \& Ducci, L. 2012, A\&A, 546, A80

Schlafly, E. F., \& Finkbeiner, D. P. 2011, ApJ, 737, 103

Schlegel, D. J., Finkbeiner, D. P., \& Davis, M. 1998, ApJ, 500, 525

Shigeyama, T., Suzuki, T., Kumagai, S., et al. 1994, ApJ, 420, 341

Skrutskie, M. F., Cutri, R. M., Stiening, R., et al. 2006, AJ, 131, 1163

Smartt, S. J., Eldridge, J. J., Crockett, R. M., \& Maund, J. R. 2009, MNRAS, 395, 1409

Sobolev, V. V. 1957, SvA, 1, 678

Soderberg, A. M., Margutti, R., Zauderer, B. A., et al. 2012, ApJ, 752, 78

Stancliffe, R. J., \& Eldridge, J. J. 2009, MNRAS, 396, 1699

Stanishev, V. 2007, Astron. Nachr., 328, 948

Stritzinger, M., Hamuy, M., Suntzeff, N. B., et al. 2002, AJ, 124, 2100

Szczygieł, D. M., Gerke, J. R., Kochanek, C. S., \& Stanek, K. Z. 2012, ApJ, 747, 23

Takáts, K., \& Vinkó, J. 2006, MNRAS, 372, 1735

Taubenberger, S., Navasardyan, H., Maurer, J. I., et al. 2011, MNRAS, 413, 2140

Terry, J. N., Paturel, G., \& Ekholm, T. 2002, A\&A, 393, 57

Theureau, G., Hanski, M. O., Coudreau, N., Hallet, N., \& Martin, J.-M. 2007, A\&A, 465, 71

Tonry, J. L., Dressler, A., Blakeslee, J. P., et al. 2001, ApJ, 546, 681

Tsvetkov, D. Y., Volkov, I. M., Baklanov, P., Blinnikov, S., \& Tuchin, O. 2009, Peremennye Zvezdy, 29, 2

Tsvetkov, D. Y., Volkov, I. M., Sorokina, E., et al. 2012, Peremennye Zvezdy, 32,6

Tully, R. B. 1988, Nearby galaxies catalog (Cambridge: Cambridge University Press)

Turatto, M., Benetti, S., \& Cappellaro, E. 2003, in From Twilight to Highlight: The Physics of Supernovae, eds. W. Hillebrandt, \& B. Leibundgut, 200

Valenti, S., Fraser, M., Benetti, S., et al. 2011, MNRAS, 416, 3138

Van Dyk, S. D., Li, W., Cenko, S. B., et al. 2011, ApJ, 741, L28

Van Dyk, S. D., Zheng, W., Clubb, K. I., et al. 2013, ApJ, 772, L32

Vinkó, J., Takáts, K., Szalai, T., et al. 2012, A\&A, 540, A93

Wada, T., \& Ueno, M. 1997, AJ, 113, 231

Woosley, S. E., Eastman, R. G., Weaver, T. A., \& Pinto, P. A. 1994, ApJ, 429, 300

Yaron, O., \& Gal-Yam, A. 2012, PASP, 124, 668 




Fig. 2. Optical and NIR (interpolated) spectral evolution for SN 2011dh for days 5-100 with a 5 day sampling. Telluric absorption bands are marked with $\mathrm{a} \oplus$ symbol in the optical and shown as grey regions in the NIR. 


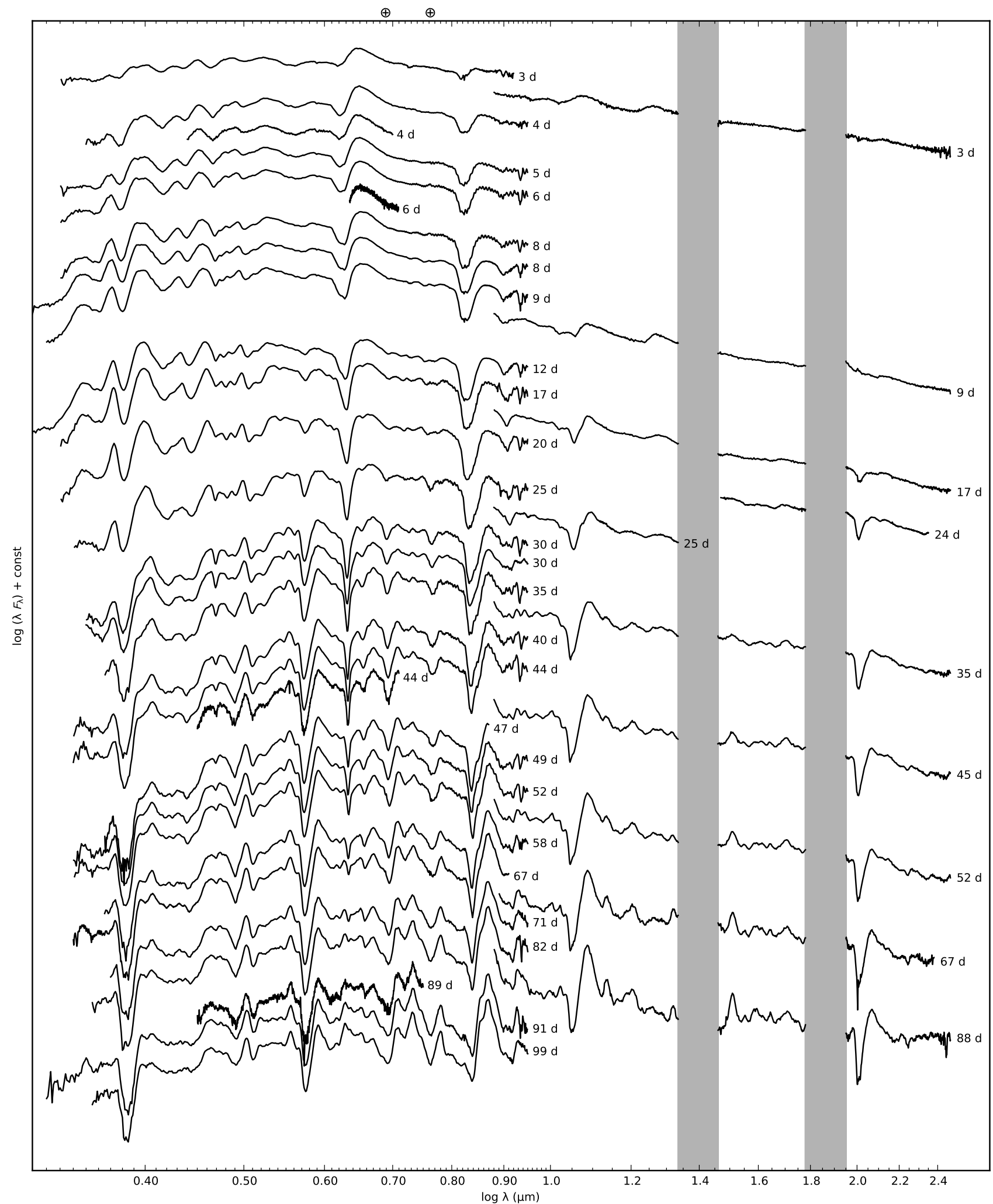

Fig. 3. Sequence of the observed spectra for SN 2011dh. Spectra obtained on the same night using the same telescope and instrument have been combined and each spectra have been labelled with the phase of the SN. Telluric absorption bands are marked with a $\oplus$ symbol in the optical and shown as grey regions in the NIR. 
A\&A 562, A17 (2014)

Table 3. Optical colour-corrected JC $U$ and S-corrected JC BVRI magnitudes for SN 2011dh.

\begin{tabular}{|c|c|c|c|c|c|c|c|}
\hline $\begin{array}{l}\mathrm{JD}(+2400000) \\
\text { (d) }\end{array}$ & $\begin{array}{l}\text { Phase } \\
\text { (d) }\end{array}$ & $\begin{array}{c}U \\
(\mathrm{mag})\end{array}$ & $\begin{array}{c}B \\
(\mathrm{mag})\end{array}$ & $\begin{array}{c}V \\
(\mathrm{mag})\end{array}$ & $\begin{array}{c}R \\
(\mathrm{mag})\end{array}$ & $\begin{array}{c}I \\
(\mathrm{mag})\end{array}$ & Telescope (Instrument) \\
\hline 55716.43 & 3.43 & $14.99(0.03)$ & $15.35(0.02)$ & $14.92(0.02)$ & $14.55(0.01)$ & $14.42(0.02)$ & LT (RATCam) \\
\hline 55716.43 & 3.43 & $15.15(0.08)$ & $15.39(0.02)$ & $14.95(0.02)$ & $14.58(0.01)$ & $14.47(0.01)$ & TNG (LRS) \\
\hline 55717.43 & 4.43 & $15.03(0.03)$ & $15.14(0.02)$ & $14.67(0.03)$ & $14.26(0.01)$ & $14.26(0.03)$ & LT (RATCam) \\
\hline 55717.48 & 4.48 & $15.17(0.09)$ & $15.21(0.03)$ & $14.63(0.03)$ & $14.24(0.01)$ & $14.23(0.02)$ & AS-1.82 m (AFOSC) \\
\hline 55717.48 & 4.48 & $\ldots$ & $15.12(0.03)$ & $14.63(0.02)$ & $14.28(0.01)$ & $14.28(0.02)$ & CANTAB (BIGST8) \\
\hline 55718.48 & 5.48 & $\ldots$ & $14.84(0.01)$ & $14.28(0.02)$ & $13.95(0.01)$ & $13.94(0.01)$ & LT (RATCam) \\
\hline 55718.57 & 5.57 & $14.68(0.06)$ & $14.84(0.02)$ & $14.24(0.02)$ & $13.92(0.01)$ & $14.05(0.01)$ & CA-2.2 m (CAFOS) \\
\hline 55720.42 & 7.42 & $14.42(0.02)$ & $14.25(0.01)$ & $13.75(0.03)$ & $13.42(0.01)$ & $13.43(0.02)$ & LT (RATCam) \\
\hline 55721.42 & 8.42 & $14.28(0.10)$ & $14.02(0.01)$ & $13.48(0.01)$ & $13.23(0.01)$ & $13.24(0.02)$ & LT (RATCam) \\
\hline 55721.43 & 8.43 & $14.07(0.07)$ & $14.06(0.01)$ & $13.60(0.04)$ & $13.27(0.02)$ & $13.34(0.02)$ & NOT (ALFOSC) \\
\hline 55722.42 & 9.42 & $\ldots$ & $13.86(0.01)$ & $13.29(0.01)$ & $13.06(0.01)$ & $13.07(0.01)$ & LT (RATCam) \\
\hline 55723.41 & 10.41 & $13.98(0.06)$ & $13.71(0.01)$ & $13.16(0.01)$ & $12.89(0.01)$ & $12.89(0.01)$ & LT (RATCam) \\
\hline 55724.41 & 11.41 & $13.91(0.08)$ & $13.61(0.01)$ & $13.03(0.01)$ & $12.80(0.01)$ & $12.77(0.01)$ & LT (RATCam) \\
\hline 55725.39 & 12.39 & $\ldots$ & $\ldots$ & $12.94(0.02)$ & $12.67(0.01)$ & $\ldots$ & MONTCAB (BIGST8) \\
\hline 55725.43 & 12.43 & $13.88(0.07)$ & $13.52(0.02)$ & $12.92(0.04)$ & $12.68(0.01)$ & $12.68(0.01)$ & LT (RATCam) \\
\hline 55726.36 & 13.36 & $\ldots$ & $13.52(0.01)$ & $12.91(0.02)$ & $12.60(0.01)$ & $\ldots$ & MONTCAB (BIGST8) \\
\hline 55728.40 & 15.40 & $\ldots$ & $13.39(0.01)$ & $12.78(0.01)$ & $12.45(0.01)$ & $\ldots$ & MONTCAB (BIGST8) \\
\hline 55729.39 & 16.39 & $13.65(0.01)$ & $13.34(0.01)$ & $12.77(0.06)$ & $12.40(0.01)$ & $12.34(0.02)$ & LT (RATCam) \\
\hline 55730.40 & 17.40 & $13.64(0.03)$ & $13.33(0.01)$ & $12.66(0.01)$ & $12.37(0.01)$ & $12.32(0.01)$ & LT (RATCam) \\
\hline 55731.41 & 18.41 & $13.74(0.09)$ & $13.30(0.01)$ & $12.60(0.02)$ & $12.32(0.01)$ & $12.27(0.01)$ & LT (RATCam) \\
\hline 55731.82 & 18.82 & $\ldots$ & $\ldots$ & $\ldots$ & $12.33(0.02)$ & $12.25(0.01)$ & FTN (FS02) \\
\hline 55732.40 & 19.40 & $\ldots$ & $13.35(0.03)$ & $12.61(0.01)$ & $12.28(0.01)$ & $12.21(0.01)$ & CANTAB (BIGST8) \\
\hline 55732.41 & 19.41 & $13.44(0.06)$ & $13.36(0.02)$ & $12.64(0.02)$ & $12.34(0.02)$ & $12.31(0.02)$ & NOT (ALFOSC) \\
\hline 55732.46 & 19.46 & $13.71(0.07)$ & $13.31(0.01)$ & $12.58(0.01)$ & $12.29(0.02)$ & $12.22(0.01)$ & LT (RATCam) \\
\hline 55733.45 & 20.45 & $13.67(0.07)$ & $\ldots$ & $\ldots$ & $12.27(0.01)$ & $12.20(0.02)$ & LT (RATCam) \\
\hline 55734.52 & 21.52 & $13.37(0.05)$ & $13.33(0.01)$ & $12.58(0.01)$ & $12.26(0.01)$ & $12.29(0.01)$ & CA-2.2 m (CAFOS) \\
\hline 55735.44 & 22.44 & $13.91(0.04)$ & $\ldots$ & $\ldots$ & $12.27(0.01)$ & $12.15(0.01)$ & LT (RATCam) \\
\hline 55736.44 & 23.44 & $14.13(0.08)$ & $\ldots$ & $\ldots$ & $12.27(0.01)$ & $12.16(0.01)$ & LT (RATCam) \\
\hline 55737.39 & 24.39 & $\ldots$ & $13.65(0.01)$ & $12.73(0.01)$ & $\ldots$ & $\ldots$ & LT (RATCam) \\
\hline 55738.42 & 25.42 & $14.50(0.04)$ & $13.78(0.02)$ & $12.81(0.01)$ & $12.33(0.02)$ & $12.22(0.01)$ & LT (RATCam) \\
\hline 55738.51 & 25.51 & $14.20(0.04)$ & $13.77(0.02)$ & $12.83(0.01)$ & $12.39(0.01)$ & $12.26(0.01)$ & NOT (ALFOSC) \\
\hline 55739.43 & 26.43 & $14.73(0.04)$ & $13.95(0.02)$ & $12.88(0.01)$ & $12.39(0.01)$ & $12.23(0.01)$ & LT (RATCam) \\
\hline 55740.36 & 27.36 & $\ldots$ & $14.08(0.04)$ & $12.94(0.01)$ & $12.46(0.01)$ & $12.29(0.01)$ & MONTCAB (BIGST8) \\
\hline 55740.43 & 27.43 & $14.91(0.03)$ & $14.11(0.01)$ & $12.98(0.01)$ & $12.49(0.01)$ & $12.29(0.01)$ & LT (RATCam) \\
\hline 55740.44 & 27.44 & $\ldots$ & $\ldots$ & $12.97(0.01)$ & $12.48(0.01)$ & $\ldots$ & TJO (MEIA) \\
\hline 55741.44 & 28.44 & $\ldots$ & $\ldots$ & $\ldots$ & $12.55(0.01)$ & $12.31(0.01)$ & LT (RATCam) \\
\hline 55742.49 & 29.49 & $15.33(0.01)$ & $\ldots$ & $\ldots$ & $12.63(0.01)$ & $12.40(0.01)$ & LT (RATCam) \\
\hline 55743.41 & 30.41 & $\ldots$ & $14.52(0.01)$ & $13.27(0.02)$ & $\ldots$ & $\ldots$ & LT (RATCam) \\
\hline 55743.42 & 30.42 & $15.18(0.05)$ & $14.51(0.02)$ & $\ldots$ & $12.66(0.01)$ & $12.53(0.01)$ & CA-2.2 m (CAFOS) \\
\hline 55743.42 & 30.42 & $15.43(0.05)$ & $14.52(0.01)$ & $13.26(0.03)$ & $12.69(0.01)$ & $12.49(0.01)$ & NOT (ALFOSC) \\
\hline 55745.39 & 32.39 & $15.74(0.03)$ & $14.73(0.01)$ & $13.44(0.01)$ & $12.79(0.01)$ & $12.56(0.01)$ & NOT (ALFOSC) \\
\hline 55745.44 & 32.44 & $15.93(0.04)$ & $\ldots$ & $\ldots$ & $12.82(0.01)$ & $12.53(0.01)$ & LT (RATCam) \\
\hline 55745.80 & 32.80 & $\ldots$ & $\ldots$ & $\ldots$ & $12.80(0.01)$ & $12.51(0.01)$ & FTN (FS02) \\
\hline 55746.45 & 33.45 & $16.07(0.04)$ & $14.86(0.03)$ & $13.51(0.01)$ & $12.84(0.01)$ & $12.55(0.02)$ & LT (RATCam) \\
\hline 55747.44 & 34.44 & $16.12(0.04)$ & $\ldots$ & $\ldots$ & $12.89(0.01)$ & $12.59(0.01)$ & LT (RATCam) \\
\hline 55748.43 & 35.43 & $16.02(0.02)$ & $14.95(0.01)$ & $13.63(0.01)$ & $12.90(0.01)$ & $12.65(0.01)$ & NOT (ALFOSC) \\
\hline 55748.44 & 35.44 & $16.27(0.04)$ & ... & $\ldots$ & $12.95(0.01)$ & $12.62(0.01)$ & LT (RATCam) \\
\hline 55750.40 & 37.40 & $16.20(0.04)$ & $15.09(0.01)$ & $13.74(0.01)$ & $13.04(0.01)$ & $12.73(0.01)$ & NOT (ALFOSC) \\
\hline 55750.42 & 37.42 & $16.41(0.14)$ & $15.10(0.02)$ & $13.78(0.03)$ & $13.04(0.01)$ & $12.73(0.02)$ & LT (RATCam) \\
\hline 55751.41 & 38.41 & $\ldots$ & $15.13(0.01)$ & $13.81(0.01)$ & $13.10(0.01)$ & $12.73(0.01)$ & TJO (MEIA) \\
\hline 55751.43 & 38.43 & $\ldots$ & $\ldots$ & $\ldots$ & $13.12(0.01)$ & $12.76(0.01)$ & LT (RATCam) \\
\hline 55752.45 & 39.45 & $16.54(0.16)$ & $\ldots$ & $\ldots$ & $13.14(0.01)$ & $12.74(0.01)$ & LT (RATCam) \\
\hline 55753.42 & 40.42 & $\ldots$ & $15.28(0.01)$ & $13.91(0.02)$ & $\ldots$ & $\ldots$ & LT (RATCam) \\
\hline 55753.46 & 40.46 & $16.45(0.05)$ & $15.23(0.01)$ & $13.86(0.01)$ & $13.17(0.01)$ & $12.81(0.01)$ & NOT (ALFOSC) \\
\hline 55755.40 & 42.40 & $16.42(0.04)$ & $15.29(0.01)$ & $13.97(0.01)$ & $13.24(0.01)$ & $12.89(0.01)$ & NOT (ALFOSC) \\
\hline 55756.44 & 43.44 & $\ldots$ & $15.26(0.02)$ & $13.99(0.02)$ & $13.30(0.02)$ & $12.86(0.01)$ & AS-Schmidt (SBIG) \\
\hline 55756.45 & 43.45 & $\ldots$ & $15.37(0.02)$ & $13.98(0.01)$ & $13.28(0.03)$ & $12.92(0.01)$ & LT (RATCam) \\
\hline 55757.43 & 44.43 & $16.42(0.04)$ & $15.37(0.01)$ & $14.05(0.01)$ & $13.30(0.01)$ & $12.97(0.01)$ & NOT (ALFOSC) \\
\hline 55759.45 & 46.45 & ... & $15.43(0.01)$ & $14.07(0.02)$ & $\ldots$ & ... & LT (RATCam) \\
\hline 55761.40 & 48.40 & $\ldots$ & $15.42(0.01)$ & $14.18(0.01)$ & $13.46(0.01)$ & $13.03(0.01)$ & AS-Schmidt (SBIG) \\
\hline 55762.41 & 49.41 & $\ldots$ & $15.44(0.01)$ & $14.17(0.01)$ & $13.46(0.01)$ & $13.06(0.01)$ & NOT (ALFOSC) \\
\hline 55762.78 & 49.78 & $\ldots$ & $\ldots$ & $\ldots$ & $13.44(0.01)$ & $13.03(0.01)$ & FTN (FSO2) \\
\hline 55763.44 & 50.44 & $\ldots$ & $15.45(0.01)$ & $14.22(0.01)$ & $13.49(0.01)$ & $13.10(0.01)$ & AS-Schmidt (SBIG) \\
\hline 55765.43 & 52.43 & $16.44(0.03)$ & $15.51(0.01)$ & $14.26(0.01)$ & $13.57(0.01)$ & $13.17(0.01)$ & NOT (ALFOSC) \\
\hline
\end{tabular}

Notes. Errors are given in parentheses. 
Table 3. continued.

\begin{tabular}{|c|c|c|c|c|c|c|c|}
\hline $\begin{array}{l}\text { JD (+2 } 400000) \\
\text { (d) }\end{array}$ & $\begin{array}{l}\text { Phase } \\
\text { (d) }\end{array}$ & $\begin{array}{c}U \\
(\mathrm{mag})\end{array}$ & $\begin{array}{c}B \\
(\mathrm{mag})\end{array}$ & $\begin{array}{c}V \\
(\mathrm{mag})\end{array}$ & $\begin{array}{c}R \\
(\mathrm{mag})\end{array}$ & $\begin{array}{c}I \\
(\mathrm{mag}) \\
\end{array}$ & Telescope (Instrument) \\
\hline 55767.43 & 54.43 & $16.50(0.05)$ & & & $13.59(0.01)$ & $13.17(0.02)$ & LT (RATCam) \\
\hline 55768.45 & 55.45 & $16.48(0.04)$ & .. & & $13.60(0.02)$ & $13.19(0.02)$ & LT (RATCam) \\
\hline 55771.40 & 58.40 & $16.37(0.03)$ & $15.58(0.01)$ & $14.33(0.01)$ & $13.64(0.01)$ & $13.29(0.01)$ & CA-2.2 m (CAFOS) \\
\hline 55773.39 & 60.39 & $16.45(0.04)$ & $15.59(0.01)$ & $14.39(0.01)$ & $13.72(0.01)$ & $13.32(0.01)$ & NOT (ALFOSC) \\
\hline 55776.38 & 63.38 & $16.47(0.04)$ & $15.63(0.01)$ & $14.46(0.01)$ & $13.79(0.01)$ & $13.36(0.01)$ & NOT (ALFOSC) \\
\hline 55777.33 & 64.33 & & $15.50(0.03)$ & $14.46(0.02)$ & $13.79(0.02)$ & $13.35(0.02)$ & AS-Schmidt (SBIG) \\
\hline 55780.40 & 67.40 & $16.42(0.03)$ & $15.65(0.01)$ & $14.51(0.01)$ & $13.86(0.01)$ & $13.43(0.01)$ & NOT (ALFOSC) \\
\hline 55783.43 & 70.43 & $16.41(0.03)$ & $15.70(0.01)$ & $14.58(0.01)$ & $13.95(0.01)$ & $13.51(0.01)$ & NOT (ALFOSC) \\
\hline 55784.33 & 71.33 & & $15.65(0.02)$ & $14.59(0.01)$ & & $13.43(0.02)$ & AS-Schmidt (SBIG) \\
\hline 55784.39 & 71.39 & $16.45(0.04)$ & $15.66(0.01)$ & $14.52(0.02)$ & $13.91(0.01)$ & $13.48(0.02)$ & CA-2.2 m (CAFOS) \\
\hline 55784.77 & 71.77 & $\ldots$ & $\ldots$ & $\ldots$ & $13.93(0.02)$ & $13.45(0.01)$ & FTN (FS02) \\
\hline 55785.36 & 72.36 & $\ldots$ & $15.69(0.02)$ & $14.62(0.01)$ & $13.97(0.01)$ & $13.46(0.01)$ & AS-Schmidt (SBIG) \\
\hline 55788.41 & 75.41 & & $\ldots$ & $\ldots$ & $14.03(0.02)$ & $13.52(0.01)$ & AS-Schmidt (SBIG) \\
\hline 55790.38 & 77.38 & $16.45(0.09)$ & $\ldots$ & $\ldots$ & $14.04(0.01)$ & $13.61(0.01)$ & LT (RATCam) \\
\hline 55793.37 & 80.37 & $16.55(0.07)$ & $15.79(0.01)$ & $14.75(0.01)$ & $14.14(0.01)$ & $13.68(0.01)$ & NOT (ALFOSC) \\
\hline 55795.35 & 82.35 & $16.40(0.04)$ & $15.78(0.01)$ & $14.76(0.01)$ & $14.14(0.01)$ & $13.69(0.01)$ & CA-2.2 m (CAFOS) \\
\hline 55797.37 & 84.37 & $\ldots$ & $15.81(0.02)$ & $14.82(0.01)$ & & & AS-Schmidt (SBIG) \\
\hline 55797.76 & 84.76 & $\ldots$ & $\ldots$ & $\ldots$ & $14.22(0.01)$ & $13.68(0.01)$ & FTN (FS02) \\
\hline 55798.36 & 85.36 & $16.50(0.03)$ & $15.83(0.01)$ & $14.84(0.01)$ & $14.26(0.01)$ & $13.65(0.02)$ & NOT (ALFOSC) \\
\hline 55799.33 & 86.33 & & $15.81(0.01)$ & $14.87(0.01)$ & & & AS-Schmidt (SBIG) \\
\hline 55801.36 & 88.36 & $16.44(0.04)$ & $15.88(0.01)$ & $14.90(0.01)$ & $14.32(0.01)$ & $13.81(0.01)$ & NOT (ALFOSC) \\
\hline 55801.40 & 88.40 & $\ldots$ & $15.79(0.02)$ & $14.90(0.01)$ & & & AS-Schmidt (SBIG) \\
\hline 55803.35 & 90.35 & $\ldots$ & $15.86(0.02)$ & $14.91(0.01)$ & $14.34(0.01)$ & $13.80(0.01)$ & AS-Schmidt (SBIG) \\
\hline 55805.33 & 92.33 & $\ldots$ & $15.85(0.02)$ & $14.97(0.02)$ & $14.38(0.01)$ & $13.84(0.01)$ & AS-Schmidt (SBIG) \\
\hline 55810.34 & 97.34 & $16.68(0.06)$ & $15.99(0.01)$ & $15.11(0.01)$ & $14.53(0.01)$ & $14.03(0.01)$ & NOT (ALFOSC) \\
\hline 55812.33 & 99.33 & $16.51(0.03)$ & $16.01(0.01)$ & $15.06(0.01)$ & $14.51(0.01)$ & $14.01(0.01)$ & CA-2.2 m (CAFOS) \\
\hline
\end{tabular}


Table 4. Optical S-corrected Swift JC UBV magnitudes for SN 2011dh.

\begin{tabular}{|c|c|c|c|c|c|}
\hline $\begin{array}{l}\text { JD }(+2400000) \\
\text { (d) }\end{array}$ & $\begin{array}{l}\text { Phase } \\
\text { (d) }\end{array}$ & $\begin{array}{c}U \\
(\mathrm{mag})\end{array}$ & $\begin{array}{c}B \\
(\mathrm{mag})\end{array}$ & $\begin{array}{c}V \\
(\mathrm{mag})\end{array}$ & Telescope (Instrument) \\
\hline 55716.01 & 3.01 & $14.92(0.02)$ & $15.35(0.02)$ & $14.96(0.02)$ & Swift (UVOT) \\
\hline 55716.68 & 3.68 & $15.05(0.02)$ & $15.29(0.02)$ & $14.90(0.01)$ & Swift (UVOT) \\
\hline 55717.82 & 4.82 & $15.03(0.04)$ & $15.03(0.03)$ & $14.57(0.03)$ & Swift (UVOT) \\
\hline 55719.03 & 6.03 & $14.77(0.03)$ & $14.65(0.02)$ & $14.14(0.02)$ & Swift (UVOT) \\
\hline 55720.83 & 7.83 & $14.31(0.03)$ & $14.15(0.02)$ & $13.65(0.01)$ & Swift (UVOT) \\
\hline 55721.84 & 8.84 & $14.11(0.02)$ & $13.96(0.02)$ & $13.43(0.01)$ & Swift (UVOT) \\
\hline 55723.18 & 10.18 & $13.93(0.02)$ & $13.73(0.02)$ & $13.20(0.01)$ & Swift (UVOT) \\
\hline 55723.98 & 10.98 & $13.85(0.02)$ & $13.68(0.02)$ & $13.12(0.01)$ & Swift (UVOT) \\
\hline 55725.13 & 12.13 & $13.77(0.02)$ & $13.57(0.02)$ & $12.97(0.01)$ & Swift (UVOT) \\
\hline 55726.66 & 13.66 & $13.78(0.02)$ & $13.57(0.02)$ & $12.91(0.01)$ & Swift (UVOT) \\
\hline 55727.79 & 14.79 & $13.71(0.02)$ & $13.48(0.02)$ & $12.80(0.01)$ & Swift (UVOT) \\
\hline 55727.87 & 14.87 & $13.76(0.05)$ & $\ldots$ & $\ldots$ & Swift (UVOT) \\
\hline 55729.25 & 16.25 & $13.69(0.02)$ & $13.42(0.02)$ & $12.77(0.01)$ & Swift (UVOT) \\
\hline 55729.60 & 16.60 & $13.63(0.02)$ & $13.42(0.02)$ & $12.72(0.01)$ & Swift (UVOT) \\
\hline 55730.53 & 17.53 & $13.66(0.02)$ & $13.36(0.02)$ & $12.69(0.01)$ & Swift (UVOT) \\
\hline 55731.65 & 18.65 & $13.66(0.02)$ & $13.35(0.02)$ & $12.64(0.01)$ & Swift (UVOT) \\
\hline 55732.67 & 19.67 & $13.69(0.02)$ & $13.37(0.02)$ & $12.62(0.01)$ & Swift (UVOT) \\
\hline 55733.89 & 20.89 & $13.74(0.02)$ & $13.37(0.02)$ & $12.63(0.01)$ & Swift (UVOT) \\
\hline 55734.75 & 21.75 & $13.82(0.02)$ & $13.44(0.02)$ & $12.63(0.01)$ & Swift (UVOT) \\
\hline 55735.95 & 22.95 & $13.99(0.03)$ & $13.53(0.02)$ & $12.68(0.01)$ & Swift (UVOT) \\
\hline 55736.55 & 23.55 & $14.05(0.03)$ & $13.55(0.02)$ & $12.71(0.01)$ & Swift (UVOT) \\
\hline 55737.55 & 24.55 & $14.30(0.03)$ & $13.70(0.02)$ & $12.77(0.01)$ & Swift (UVOT) \\
\hline 55738.76 & 25.76 & $14.54(0.03)$ & $13.83(0.02)$ & $12.84(0.02)$ & Swift (UVOT) \\
\hline 55740.28 & 27.28 & $14.85(0.03)$ & $14.07(0.02)$ & $13.01(0.03)$ & Swift (UVOT) \\
\hline 55741.37 & 28.37 & $15.08(0.05)$ & $14.23(0.03)$ & $13.09(0.02)$ & Swift (UVOT) \\
\hline 55741.77 & 28.77 & $15.20(0.05)$ & $14.32(0.03)$ & $13.16(0.02)$ & Swift (UVOT) \\
\hline 55742.84 & 29.84 & $15.43(0.05)$ & $14.46(0.03)$ & $13.26(0.02)$ & Swift (UVOT) \\
\hline 55743.84 & 30.84 & $15.63(0.05)$ & $14.55(0.03)$ & $13.33(0.02)$ & Swift (UVOT) \\
\hline 55745.25 & 32.25 & $15.76(0.05)$ & $14.74(0.03)$ & $13.43(0.02)$ & Swift (UVOT) \\
\hline 55746.12 & 33.12 & $15.91(0.06)$ & $14.80(0.03)$ & $13.50(0.02)$ & Swift (UVOT) \\
\hline 55750.60 & 37.60 & $16.30(0.06)$ & $15.03(0.03)$ & $13.74(0.02)$ & Swift (UVOT) \\
\hline 55754.62 & 41.62 & $16.46(0.07)$ & $15.24(0.03)$ & $13.97(0.02)$ & Swift (UVOT) \\
\hline 55758.55 & 45.55 & $16.54(0.08)$ & $15.37(0.03)$ & $14.07(0.02)$ & Swift (UVOT) \\
\hline 55762.57 & 49.57 & $16.75(0.10)$ & $15.45(0.04)$ & $14.19(0.02)$ & Swift (UVOT) \\
\hline 55766.52 & 53.52 & $16.60(0.08)$ & $15.57(0.04)$ & $14.30(0.02)$ & Swift (UVOT) \\
\hline 55770.80 & 57.80 & $16.48(0.07)$ & $15.61(0.04)$ & $14.35(0.02)$ & Swift (UVOT) \\
\hline 55775.69 & 62.69 & $16.42(0.07)$ & $15.60(0.04)$ & $14.48(0.03)$ & Swift (UVOT) \\
\hline 55780.50 & 67.50 & $16.60(0.08)$ & $15.70(0.04)$ & $14.52(0.03)$ & Swift (UVOT) \\
\hline 55784.80 & 71.80 & $16.46(0.07)$ & $15.71(0.04)$ & $14.61(0.03)$ & Swift (UVOT) \\
\hline 55788.74 & 75.74 & $16.43(0.06)$ & $15.73(0.04)$ & $14.64(0.03)$ & Swift (UVOT) \\
\hline
\end{tabular}

Notes. Errors are given in parentheses. 
M. Ergon et al.: SN 2011dh - The first 100 days

Table 5. Optical colour-corrected SDSS $u$ and S-corrected SDSS griz magnitudes for SN 2011dh.

\begin{tabular}{|c|c|c|c|c|c|c|c|}
\hline $\begin{array}{l}\text { JD (+2 } 400000) \\
\text { (d) }\end{array}$ & $\begin{array}{l}\text { Phase } \\
\text { (d) }\end{array}$ & $\begin{array}{c}u \\
(\mathrm{mag})\end{array}$ & $\begin{array}{c}g \\
(\mathrm{mag})\end{array}$ & $\begin{array}{c}r \\
(\mathrm{mag})\end{array}$ & $\begin{array}{c}i \\
(\mathrm{mag})\end{array}$ & $\begin{array}{c}z \\
(\mathrm{mag})\end{array}$ & Telescope (Instrument) \\
\hline 55716.47 & 3.47 & $15.79(0.03)$ & $15.08(0.01)$ & $14.69(0.01)$ & $14.80(0.01)$ & $14.76(0.02)$ & LT (RATCam) \\
\hline 55717.46 & 4.46 & $15.89(0.03)$ & $14.80(0.01)$ & $14.39(0.01)$ & $14.61(0.01)$ & $14.58(0.02)$ & LT (RATCam) \\
\hline 55718.53 & 5.53 & $\ldots$ & $14.44(0.04)$ & $14.06(0.01)$ & $14.27(0.01)$ & $\ldots$ & LT (RATCam) \\
\hline 55720.44 & 7.44 & $15.26(0.02)$ & $13.97(0.01)$ & $13.53(0.01)$ & $13.72(0.02)$ & $13.87(0.01)$ & LT (RATCam) \\
\hline 55721.44 & 8.44 & $14.96(0.01)$ & $13.78(0.01)$ & $13.34(0.01)$ & $13.51(0.01)$ & $13.64(0.01)$ & LT (RATCam) \\
\hline 55722.44 & 9.44 & & $13.59(0.01)$ & $13.18(0.01)$ & $13.34(0.01)$ & $13.49(0.01)$ & LT (RATCam) \\
\hline 55723.41 & 10.41 & $14.67(0.03)$ & - & $13.03(0.01)$ & $13.16(0.01)$ & $13.34(0.01)$ & LT (RATCam) \\
\hline 55724.41 & 11.41 & $14.56(0.02)$ &  & $12.93(0.01)$ & $13.05(0.01)$ & $13.22(0.01)$ & LT (RATCam) \\
\hline 55725.43 & 12.43 & $14.57(0.04)$ & $\ldots$ & $12.83(0.01)$ & $12.93(0.01)$ & $13.10(0.01)$ & LT (RATCam) \\
\hline 55729.39 & 16.39 & $14.40(0.03)$ & $13.10(0.01)$ & $12.56(0.01)$ & $12.61(0.01)$ & $12.81(0.01)$ & LT (RATCam) \\
\hline 55730.40 & 17.40 & $14.29(0.03)$ & $13.07(0.01)$ & $12.51(0.01)$ & $12.56(0.01)$ & $12.78(0.01)$ & LT (RATCam) \\
\hline 55731.41 & 18.41 & $14.38(0.03)$ & $13.02(0.01)$ & $12.46(0.01)$ & $12.50(0.01)$ & $12.72(0.01)$ & LT (RATCam) \\
\hline 55731.82 & 18.82 & & $13.07(0.01)$ & $12.46(0.01)$ & $12.50(0.01)$ & $12.65(0.01)$ & FTN (FSO2) \\
\hline 55732.46 & 19.46 & $14.40(0.01)$ & $12.99(0.03)$ & $12.43(0.01)$ & $12.47(0.01)$ & $12.68(0.01)$ & LT (RATCam) \\
\hline 55733.45 & 20.45 & $14.36(0.05)$ & $13.02(0.01)$ & $12.42(0.01)$ & $12.44(0.01)$ & $12.65(0.01)$ & LT (RATCam) \\
\hline 55735.44 & 22.44 & $14.60(0.04)$ & $13.12(0.01)$ & $12.43(0.01)$ & $12.41(0.01)$ & $12.60(0.01)$ & LT (RATCam) \\
\hline 55736.44 & 23.44 & $14.81(0.03)$ & $13.19(0.02)$ & $12.46(0.01)$ & $12.41(0.01)$ & $12.59(0.02)$ & LT (RATCam) \\
\hline 55738.45 & 25.45 & $15.23(0.02)$ & $13.43(0.01)$ & $12.56(0.01)$ & $12.46(0.01)$ & $12.66(0.01)$ & LT (RATCam) \\
\hline 55739.44 & 26.44 & $15.41(0.02)$ & $13.50(0.03)$ & $12.60(0.01)$ & $12.50(0.01)$ & $12.65(0.01)$ & LT (RATCam) \\
\hline 55740.44 & 27.44 & $15.67(0.01)$ & $13.65(0.01)$ & $12.67(0.01)$ & $12.54(0.01)$ & $12.70(0.01)$ & LT (RATCam) \\
\hline 55741.44 & 28.44 & & $13.75(0.02)$ & $12.77(0.01)$ & $12.58(0.02)$ & $12.76(0.01)$ & LT (RATCam) \\
\hline 55742.49 & 29.49 & $16.07(0.02)$ & $13.92(0.01)$ & $12.84(0.01)$ & $12.65(0.01)$ & $12.81(0.01)$ & LT (RATCam) \\
\hline 55745.44 & 32.44 & $16.59(0.05)$ & $14.20(0.02)$ & $13.04(0.01)$ & $12.78(0.01)$ & $12.87(0.03)$ & LT (RATCam) \\
\hline 55745.80 & 32.80 & & $14.35(0.04)$ & $13.00(0.01)$ & $12.79(0.01)$ & $12.94(0.01)$ & FTN (FS02) \\
\hline 55746.45 & 33.45 & $16.71(0.04)$ & $14.31(0.01)$ & $13.10(0.01)$ & $12.81(0.01)$ & $12.94(0.01)$ & LT (RATCam) \\
\hline 55747.44 & 34.44 & $16.78(0.04)$ & $14.40(0.02)$ & $13.14(0.01)$ & $12.85(0.01)$ & $12.96(0.01)$ & LT (RATCam) \\
\hline 55748.44 & 35.44 & $16.98(0.04)$ & $14.42(0.02)$ & $13.20(0.01)$ & $12.90(0.01)$ & $13.01(0.01)$ & LT (RATCam) \\
\hline 55750.44 & 37.44 & $17.09(0.10)$ & $14.55(0.02)$ & $13.30(0.01)$ & $13.01(0.02)$ & $13.05(0.04)$ & LT (RATCam) \\
\hline 55751.43 & 38.43 & $17.04(0.03)$ & $14.64(0.03)$ & $13.36(0.01)$ & $13.03(0.01)$ & $13.12(0.01)$ & LT (RATCam) \\
\hline 55752.45 & 39.45 & $17.14(0.07)$ & $14.65(0.01)$ & $13.40(0.01)$ & $13.03(0.01)$ & $13.10(0.01)$ & LT (RATCam) \\
\hline 55756.46 & 43.46 & $\ldots$ & $14.79(0.01)$ & $13.56(0.01)$ & $13.22(0.01)$ & $13.19(0.01)$ & LT (RATCam) \\
\hline 55762.78 & 49.78 & & $15.00(0.02)$ & $13.68(0.01)$ & $13.37(0.01)$ & $13.28(0.01)$ & FTN (FSO2) \\
\hline 55767.43 & 54.43 & $17.22(0.02)$ & $15.02(0.01)$ & $13.84(0.01)$ & $13.52(0.01)$ & $13.38(0.02)$ & LT (RATCam) \\
\hline 55768.45 & 55.45 & $17.20(0.02)$ & $15.02(0.01)$ & $13.87(0.01)$ & $13.56(0.01)$ & $13.41(0.01)$ & LT (RATCam) \\
\hline 55773.39 & 60.39 & $17.21(0.04)$ & $15.06(0.01)$ & $14.00(0.01)$ & $13.72(0.01)$ & $13.53(0.02)$ & NOT (ALFOSC) \\
\hline 55776.38 & 63.38 & $17.30(0.03)$ & $15.12(0.01)$ & $14.04(0.01)$ & $13.76(0.01)$ & $13.55(0.01)$ & NOT (ALFOSC) \\
\hline 55780.41 & 67.41 & $17.27(0.03)$ & $15.16(0.01)$ & $14.10(0.01)$ & $13.84(0.01)$ & $13.60(0.01)$ & NOT (ALFOSC) \\
\hline 55783.44 & 70.44 & $17.21(0.04)$ & $15.18(0.01)$ & $14.20(0.01)$ & $13.94(0.01)$ & $13.66(0.01)$ & NOT (ALFOSC) \\
\hline 55784.77 & 71.77 & & $15.23(0.02)$ & $14.16(0.01)$ & $13.88(0.01)$ & $13.64(0.01)$ & FTN (FS02) \\
\hline 55790.38 & 77.38 & $17.22(0.03)$ & $15.34(0.04)$ & $14.29(0.01)$ & $14.04(0.01)$ & $13.69(0.02)$ & LT (RATCam) \\
\hline 55793.37 & 80.37 & $17.27(0.03)$ & $15.30(0.01)$ & $14.40(0.01)$ & $14.16(0.01)$ & $13.83(0.01)$ & NOT (ALFOSC) \\
\hline 55797.76 & 84.76 & & $15.38(0.01)$ & $14.42(0.01)$ & $14.18(0.01)$ & $13.82(0.01)$ & FTN (FS02) \\
\hline 55798.37 & 85.37 & $17.29(0.03)$ & $15.37(0.01)$ & $14.51(0.01)$ & $14.26(0.01)$ & $13.86(0.01)$ & NOT (ALFOSC) \\
\hline 55801.36 & 88.36 & $17.28(0.01)$ & $15.41(0.01)$ & $14.54(0.01)$ & $14.31(0.01)$ & $13.88(0.01)$ & NOT (ALFOSC) \\
\hline 55810.34 & 97.34 & $17.43(0.02)$ & $15.55(0.01)$ & $14.76(0.01)$ & $14.56(0.01)$ & $14.09(0.02)$ & NOT (ALFOSC) \\
\hline
\end{tabular}

Notes. Errors are given in parentheses. 
Table 6. NIR S-corrected 2MASS JHK magnitudes for SN $2011 \mathrm{dh}$.

\begin{tabular}{lccccl}
\hline \hline $\begin{array}{l}\text { JD }(+2400000) \\
(\mathrm{d})\end{array}$ & $\begin{array}{c}\text { Phase } \\
(\mathrm{d})\end{array}$ & $\begin{array}{c}J \\
(\mathrm{mag})\end{array}$ & $\begin{array}{c}H \\
(\mathrm{mag})\end{array}$ & $\begin{array}{c}K \\
(\mathrm{mag})\end{array}$ & Telescope (Instrument) \\
\hline 55716.51 & 3.51 & $14.09(0.01)$ & $13.90(0.01)$ & $13.68(0.02)$ & TNG (NICS) \\
55722.40 & 9.40 & $12.89(0.01)$ & $12.87(0.01)$ & $12.67(0.01)$ & TNG (NICS) \\
55725.50 & 12.50 & $12.61(0.04)$ & $12.54(0.01)$ & $12.43(0.02)$ & NOT (NOTCAM) \\
55730.51 & 17.51 & $12.12(0.01)$ & $12.08(0.01)$ & $11.94(0.01)$ & TNG (NICS) \\
55737.72 & 24.72 & $11.96(0.01)$ & $11.90(0.01)$ & $11.72(0.03)$ & LBT (LUCIFER) \\
55741.13 & 28.13 & $11.94(0.01)$ & $11.90(0.02)$ & $11.70(0.05)$ & TCS (CAIN) \\
55748.43 & 35.43 & $12.14(0.01)$ & $12.00(0.02)$ & $11.77(0.01)$ & TCS (CAIN) \\
55750.42 & 37.42 & $12.19(0.01)$ & $12.00(0.01)$ & $11.84(0.04)$ & TCS (CAIN) \\
55751.42 & 38.42 & $12.29(0.01)$ & $12.01(0.01)$ & $11.84(0.03)$ & TCS (CAIN) \\
55758.45 & 45.45 & $12.55(0.01)$ & $12.22(0.01)$ & $12.06(0.01)$ & TNG (NICS) \\
55759.41 & 46.41 & $12.49(0.03)$ & $12.22(0.03)$ & $12.11(0.04)$ & TCS (CAIN) \\
55762.41 & 49.41 & $12.57(0.01)$ & $12.26(0.01)$ & $12.17(0.03)$ & TCS (CAIN) \\
55763.42 & 50.42 & $12.62(0.02)$ & $12.27(0.04)$ & $12.25(0.06)$ & TCS (CAIN) \\
55765.45 & 52.45 & $12.79(0.01)$ & $12.38(0.01)$ & $12.23(0.01)$ & TNG (NICS) \\
55769.41 & 56.41 & $12.77(0.01)$ & $12.48(0.06)$ & $12.40(0.03)$ & TCS (CAIN) \\
55773.37 & 60.37 & $12.94(0.03)$ & $12.58(0.01)$ & $12.42(0.02)$ & TNG (NICS) \\
55774.40 & 61.40 & $12.90(0.01)$ & $12.55(0.03)$ & $12.43(0.04)$ & TCS (CAIN) \\
55776.40 & 63.40 & $13.00(0.01)$ & $12.64(0.01)$ & $12.53(0.02)$ & TCS (CAIN) \\
55781.41 & 68.41 & $13.23(0.01)$ & $12.76(0.01)$ & $12.66(0.01)$ & WHT (LIRIS) \\
55787.44 & 74.44 & $13.56(0.03)$ & $13.03(0.02)$ & $12.95(0.02)$ & NOT (NOTCAM) \\
55794.41 & 81.41 & $\ldots$ & $13.18(0.01)$ & $\ldots$ & TNG (NICS) \\
55801.36 & 88.36 & $13.90(0.02)$ & $13.41(0.02)$ & $13.17(0.01)$ & TNG (NICS) \\
55804.34 & 91.34 & $14.10(0.01)$ & $13.50(0.01)$ & $13.26(0.01)$ & CA-3.5 m (O2000) \\
\hline & & & & & \\
\hline
\end{tabular}

Notes. Errors are given in parentheses.

Table 7. MIR Spitzer $3.6 \mu \mathrm{m}$ and $4.5 \mu \mathrm{m}$ magnitudes for SN $2011 \mathrm{dh}$.

\begin{tabular}{lcccl}
\hline \hline $\begin{array}{l}\text { JD }(+2400000) \\
(\mathrm{d})\end{array}$ & $\begin{array}{c}\text { Phase } \\
(\mathrm{d})\end{array}$ & $\begin{array}{c}3.6 \mu \mathrm{m} \\
(\mathrm{mag})\end{array}$ & $\begin{array}{c}4.5 \mu \mathrm{m} \\
(\mathrm{mag})\end{array}$ & Telescope (Instrument) \\
\hline 55731.21 & 18.21 & $11.83(0.02)$ & $11.48(0.02)$ & Spitzer (IRAC) \\
55737.06 & 24.06 & $11.66(0.02)$ & $11.31(0.02)$ & Spitzer (IRAC) \\
55744.32 & 31.32 & $11.66(0.02)$ & $11.30(0.02)$ & Spitzer (IRAC) \\
55751.46 & 38.46 & $11.68(0.02)$ & $11.30(0.02)$ & Spitzer (IRAC) \\
55758.75 & 45.75 & $11.79(0.02)$ & $11.32(0.02)$ & Spitzer (IRAC) \\
55766.45 & 53.45 & $11.96(0.02)$ & $11.34(0.02)$ & Spitzer (IRAC) \\
55772.33 & 59.33 & $12.11(0.03)$ & $11.38(0.02)$ & Spitzer (IRAC) \\
55779.12 & 66.12 & $12.30(0.03)$ & $11.43(0.02)$ & Spitzer (IRAC) \\
55785.60 & 72.60 & $12.50(0.03)$ & $11.50(0.02)$ & Spitzer (IRAC) \\
55798.28 & 85.28 & $12.84(0.04)$ & $11.66(0.03)$ & Spitzer (IRAC) \\
\hline
\end{tabular}

Notes. Errors are given in parentheses. 
Table 8. UV Swift UVW1, UVM2 and UVW2 magnitudes for SN 2011dh.

\begin{tabular}{lccccc}
\hline \hline $\begin{array}{l}\text { JD }(+2400000) \\
(\mathrm{d})\end{array}$ & $\begin{array}{c}\text { Phase } \\
(\mathrm{d})\end{array}$ & $\begin{array}{c}\text { UVW1 } \\
(\mathrm{mag})\end{array}$ & $\begin{array}{c}\text { UVM2 } \\
(\mathrm{mag})\end{array}$ & $\begin{array}{c}\text { UVW2 } \\
(\mathrm{mag})\end{array}$ & Telescope (Instrument) \\
\hline 55716.01 & 3.01 & $15.40(0.04)$ & $15.99(0.04)$ & $16.40(0.04)$ & Swift (UVOT) \\
55716.68 & 3.68 & $15.56(0.04)$ & $16.27(0.05)$ & $16.69(0.04)$ & Swift (UVOT) \\
55717.55 & 4.55 & $15.80(0.05)$ & $16.59(0.08)$ & $16.91(0.08)$ & Swift (UVOT) \\
55718.90 & 5.90 & $15.85(0.04)$ & $16.77(0.04)$ & $16.98(0.05)$ & Swift (UVOT) \\
55720.60 & 7.60 & $15.75(0.04)$ & $16.89(0.09)$ & $\ldots$ & Swift (UVOT) \\
55721.30 & 8.30 & $\ldots$ & $\ldots$ & $16.95(0.07)$ & Swift (UVOT) \\
55722.50 & 9.50 & $15.62(0.04)$ & $17.28(0.08)$ & $\ldots$ & Swift (UVOT) \\
55723.10 & 10.10 & $\ldots$ & $\ldots$ & $17.02(0.13)$ & Swift (UVOT) \\
55724.80 & 11.80 & $15.66(0.04)$ & $17.37(0.08)$ & $17.00(0.05)$ & Swift (UVOT) \\
55727.10 & 14.10 & $15.59(0.04)$ & $17.61(0.09)$ & $17.11(0.05)$ & Swift (UVOT) \\
55730.66 & 17.66 & $15.56(0.03)$ & $17.72(0.06)$ & $17.00(0.04)$ & Swift (UVOT) \\
55733.70 & 20.70 & $15.65(0.03)$ & $17.93(0.07)$ & $17.14(0.04)$ & Swift (UVOT) \\
55736.57 & 23.57 & $15.87(0.04)$ & $18.16(0.09)$ & $17.32(0.05)$ & Swift (UVOT) \\
55739.95 & 26.95 & $16.42(0.04)$ & $\ldots$ & $17.82(0.08)$ & Swift (UVOT) \\
55740.70 & 27.70 & $\ldots$ & $18.49(0.19)$ & $\ldots$ & Swift (UVOT) \\
55742.27 & 29.27 & $16.67(0.06)$ & $19.06(0.26)$ & $18.36(0.13)$ & Swift (UVOT) \\
55747.73 & 34.73 & $17.18(0.06)$ & $19.11(0.20)$ & $19.03(0.19)$ & Swift (UVOT) \\
55758.17 & 45.17 & $17.64(0.08)$ & $19.37(0.25)$ & $19.47(0.25)$ & Swift (UVOT) \\
55770.83 & 57.83 & $17.67(0.08)$ & $19.74(0.33)$ & $19.97(0.40)$ & Swift (UVOT) \\
55784.97 & 71.97 & $17.77(0.09)$ & $20.22(0.58)$ & $19.91(0.40)$ & Swift (UVOT) \\
\hline
\end{tabular}

Notes. Errors are given in parentheses.

Table 9. List of optical and NIR spectroscopic observations.

\begin{tabular}{|c|c|c|c|c|c|c|}
\hline $\begin{array}{l}\text { JD (+2 } 400000) \\
\text { (d) }\end{array}$ & $\begin{array}{l}\text { Phase } \\
\text { (d) }\end{array}$ & Grism & $\begin{array}{l}\text { Range } \\
(\AA)\end{array}$ & Resolution & $\begin{array}{l}\text { Resolution } \\
\text { (A) }\end{array}$ & Telescope (Instrument) \\
\hline 55716.41 & 3.41 & LRB & $3300-8000$ & 585 & 10.0 & TNG (LRS) \\
\hline 55716.41 & 3.41 & LRR & $5300-9200$ & 714 & 10.4 & TNG (LRS) \\
\hline 55716.47 & 3.47 & IJ & $9000-14500$ & 333 & $\ldots$ & TNG (NICS) \\
\hline 55716.49 & 3.49 & HK & $14000-25000$ & 333 & $\ldots$ & TNG (NICS) \\
\hline 55717.37 & 4.37 & b200 & $3300-8700$ & $\ldots$. & 12.0 & CA-2.2 m (CAFOS $)$ \\
\hline 55717.37 & 4.37 & $\mathrm{r} 200$ & $6300-10500$ & $\ldots$ & 12.0 & CA-2.2 m (CAFOS) \\
\hline 55717.49 & 4.49 & Grism 4 & $3500-8450$ & 613 & & AS $1.82 \mathrm{~m}$ (AFOSC) \\
\hline 55718.42 & 5.42 & Grism 4 & $3200-9100$ & 355 & 16.2 & NOT (ALFOSC) \\
\hline 55718.44 & 5.44 & Grism 5 & $5000-10250$ & 415 & 16.8 & NOT (ALFOSC) \\
\hline 55719.40 & 6.40 & Grism 4 & $3200-9100$ & 355 & 16.2 & NOT (ALFOSC) \\
\hline 55719.42 & 6.42 & Grism 5 & $5000-10250$ & 415 & 16.8 & NOT (ALFOSC) \\
\hline 55719.47 & 6.47 & VPH4 & $6350-7090$ & $\ldots$ & 3.7 & AS-1.82 m (AFOSC) \\
\hline 55721.39 & 8.39 & Grism 4 & $3200-9100$ & 355 & 16.2 & NOT (ALFOSC) \\
\hline 55721.40 & 8.40 & Grism 5 & $5000-10250$ & 415 & 16.8 & NOT (ALFOSC) \\
\hline 55721.45 & 8.45 & R300B & $3200-5300$ & $\ldots$ & 4.1 & WHT (ISIS) \\
\hline 55721.45 & 8.45 & $\mathrm{R} 158 \mathrm{R}$ & $5300-10000$ & $\ldots$ & 7.7 & WHT(ISIS) \\
\hline 55722.57 & 9.57 & R300B & $3200-5300$ & $\ldots$ & 4.1 & WHT (ISIS) \\
\hline 55722.57 & 9.57 & R158R & $5300-10000$ & $\ldots$ & 7.7 & WHT (ISIS) \\
\hline 55722.42 & 9.42 & IJ & $9000-14500$ & 333 & $\ldots$ & TNG (NICS) \\
\hline 55722.46 & 9.48 & HK & $14000-25000$ & 333 & $\ldots$ & TNG (NICS) \\
\hline 55723.61 & 10.61 & VHRV & $4752-6698$ & 2181 & 2.6 & TNG (LRS) \\
\hline 55725.38 & 12.38 & R300B & $3200-5300$ & $\ldots$ & 4.1 & WHT (ISIS) \\
\hline 55725.38 & 12.38 & $\mathrm{R} 158 \mathrm{R}$ & $5300-10000$ & & 7.7 & WHT (ISIS) \\
\hline 55730.45 & 17.45 & Grism 4 & $3200-9100$ & 355 & 16.2 & NOT (ALFOSC) \\
\hline 55730.46 & 17.46 & Grism 5 & $5000-10250$ & 415 & 16.8 & NOT (ALFOSC) \\
\hline 55730.52 & 17.52 & IJ & $9000-14500$ & 333 & $\ldots$ & TNG (NICS) \\
\hline 55730.57 & 17.57 & HK & $14000-25000$ & 333 & $\ldots$ & TNG (NICS) \\
\hline 55733.37 & 20.37 & b200 & $3300-8700$ & $\ldots$ & 12.0 & CA- $2.2 \mathrm{~m}$ (CAFOS) \\
\hline 55733.37 & 20.37 & $\mathrm{r} 200$ & $6300-10500$ & $\ldots$ & 12.0 & CA-2.2m (CAFOS) \\
\hline 55733.42 & 20.42 & Grism 4 & $3200-9100$ & 355 & 16.2 & NOT (ALFOSC) \\
\hline 55733.43 & 20.43 & Grism 5 & $5000-10250$ & 415 & 16.8 & NOT (ALFOSC) \\
\hline 55737.68 & 24.68 & $200 \mathrm{H}+\mathrm{K}$ & $14900-24000$ & $1881(\mathrm{H}) / 2573(\mathrm{~K})$ & $\ldots$ & LBT (LUCIFER) \\
\hline 55738.49 & 25.49 & Grism 4 & $3200-9100$ & 355 & 16.2 & NOT (ALFOSC) \\
\hline 55738.50 & 25.50 & Grism 5 & $5000-10250$ & 415 & 16.8 & NOT (ALFOSC) \\
\hline 55738.41 & 25.41 & IJ & $9000-14500$ & 333 & $\ldots$ & TNG (NICS) \\
\hline 55743.40 & 30.40 & Grism 4 & $3200-9100$ & 355 & 16.2 & NOT (ALFOSC) \\
\hline 55743.44 & 30.44 & Grism 5 & $5000-10250$ & 415 & 16.8 & NOT (ALFOSC) \\
\hline 55743.43 & 30.43 & b200 & $3300-8700$ & $\ldots$ & 12.0 & CA-2.2 m (CAFOS) \\
\hline
\end{tabular}


Table 9. continued.

\begin{tabular}{|c|c|c|c|c|c|c|}
\hline $\begin{array}{l}\mathrm{JD}(+2400000) \\
\text { (d) }\end{array}$ & $\begin{array}{l}\text { Phase } \\
\text { (d) }\end{array}$ & Grism & $\begin{array}{l}\text { Range } \\
(\AA)\end{array}$ & Resolution & $\begin{array}{l}\text { Resolution } \\
(\AA)\end{array}$ & Telescope (Instrument) \\
\hline 55743.43 & 30.43 & r200 & $6300-10500$ & & 12.0 & CA-2.2 m (CAFOS) \\
\hline 55748.40 & 35.40 & Grism 4 & $3200-9100$ & 355 & 16.2 & NOT (ALFOSC) \\
\hline 55748.41 & 35.41 & Grism 5 & $5000-10250$ & 415 & 16.8 & NOT (ALFOSC) \\
\hline 55748.39 & 35.39 & IJ & $9000-14500$ & 333 & $\ldots$ & TNG (NICS) \\
\hline 55748.42 & 35.42 & HK & $14000-25000$ & 333 & $\ldots$ & TNG (NICS) \\
\hline 55753.41 & 40.41 & Grism 4 & $3200-9100$ & 355 & 16.2 & NOT (ALFOSC) \\
\hline 55753.43 & 40.43 & Grism 5 & $5000-10250$ & 415 & 16.8 & NOT (ALFOSC) \\
\hline 55757.39 & 44.39 & Grism 4 & $3200-9100$ & 355 & 16.2 & NOT (ALFOSC) \\
\hline 55757.41 & 44.41 & Grism 5 & $5000-10250$ & 415 & 16.8 & NOT (ALFOSC) \\
\hline 55757.41 & 44.41 & gt300 & $3200-7700$ & 555 & 9.0 & AS-1.22 m (DU440) \\
\hline 55758.39 & 45.39 & $\mathrm{IJ}$ & $9000-14500$ & 333 & $\ldots$ & TNG (NICS) \\
\hline 55758.42 & 45.42 & HK & $14000-25000$ & 333 & & TNG (NICS) \\
\hline 55760.38 & 47.38 & b200 & $3300-8700$ & $\ldots$ & $\dddot{12.0}$ & CA-2.2 m (CAFOS) \\
\hline 55762.39 & 49.39 & Grism 4 & $3200-9100$ & 355 & 16.2 & NOT (ALFOSC) \\
\hline 55762.40 & 49.40 & Grism 5 & $5000-10250$ & 415 & 16.8 & NOT (ALFOSC) \\
\hline 55765.40 & 52.40 & Grism 4 & $3200-9100$ & 355 & 16.2 & NOT (ALFOSC) \\
\hline 55765.42 & 52.42 & Grism 5 & $5000-10250$ & 415 & 16.8 & NOT (ALFOSC) \\
\hline 55765.39 & 52.39 & IJ & $9000-14500$ & 333 & $\ldots$ & TNG (NICS) \\
\hline 55765.42 & 52.42 & HK & $14000-25000$ & 333 & $\ldots$ & TNG (NICS) \\
\hline 55771.41 & 58.41 & b200 & $3300-8700$ & $\ldots$ & 12.0 & CA-2.2 m (CAFOS) \\
\hline 55771.41 & 58.41 & r200 & $6300-10500$ & $\ldots$ & 12.0 & CA-2.2 m (CAFOS) \\
\hline 55780.39 & 67.39 & Grism 4 & $3200-9100$ & 355 & 16.2 & NOT (ALFOSC) \\
\hline 55780.43 & 67.43 & zJ & $8900-15100$ & 700 & $\ldots$ & WHT (LIRIS) \\
\hline 55780.40 & 67.40 & HK & $14000-23800$ & 700 & $\ldots$ & WHT (LIRIS) \\
\hline 55784.40 & 71.40 & b200 & $3300-8700$ & $\ldots$ & 12.0 & CA-2.2 m (CAFOS) \\
\hline 55784.40 & 71.40 & r200 & $6300-10500$ & $\ldots$ & 12.0 & CA-2.2 m (CAFOS) \\
\hline 55795.39 & 82.39 & b200 & $3300-8700$ & $\ldots$ & 12.0 & CA-2.2 m (CAFOS) \\
\hline 55795.39 & 82.39 & $\mathrm{r} 200$ & $6300-10500$ & $\ldots$ & 12.0 & CA-2.2 m (CAFOS) \\
\hline 55801.37 & 88.37 & IJ & $9000-14500$ & 333 & $\ldots$ & TNG (NICS) \\
\hline 55801.40 & 88.40 & HK & $14000-25000$ & 333 & & TNG (NICS) \\
\hline 55802.37 & 89.37 & gt300 & $3200-7700$ & 396 & 12.6 & AS-1.22 m (DU440) \\
\hline 55804.36 & 91.36 & R300B & $3200-5300$ & $\ldots$ & 4.1 & WHT (ISIS) \\
\hline 55804.36 & 91.36 & $\mathrm{R} 158 \mathrm{R}$ & $5300-10000$ & $\ldots$ & 7.7 & WHT (ISIS) \\
\hline 55812.36 & 99.36 & b200 & $3300-8700$ & $\ldots$ & 12.0 & CA-2.2 m (CAFOS) \\
\hline 55812.36 & 99.36 & r200 & $6300-10500$ & $\ldots$ & 12.0 & CA-2.2 m (CAFOS) \\
\hline
\end{tabular}


Table 10. Pseudo-bolometric UV to MIR lightcurve for SN 2011dh calculated from spectroscopic and photometric data.

\begin{tabular}{|c|c|c|c|c|c|}
\hline $\begin{array}{l}\text { JD }(+2400000) \\
\text { (d) }\end{array}$ & $\begin{array}{l}\text { Phase } \\
\text { (d) }\end{array}$ & $\begin{array}{c}L \\
\left(10^{41} \mathrm{erg} \mathrm{s}^{-1}\right)\end{array}$ & $\begin{array}{c}\mathrm{JD}(+2400000) \\
(\mathrm{d})\end{array}$ & $\begin{array}{l}\text { Phase } \\
\text { (d) }\end{array}$ & $\begin{array}{c}L \\
\left(10^{41} \mathrm{erg} \mathrm{s}^{-1}\right)\end{array}$ \\
\hline 55717.00 & 4.00 & $2.90(0.01)(0.66,1.24)$ & 55765.00 & 52.00 & $5.56(0.02)(1.19,2.03)$ \\
\hline 55718.00 & 5.00 & $3.59(0.01)(0.80,1.49)$ & 55766.00 & 53.00 & $5.44(0.02)(1.16,1.99)$ \\
\hline 55719.00 & 6.00 & $4.56(0.01)(1.02,1.88)$ & 55767.00 & 54.00 & $5.32(0.02)(1.14,1.95)$ \\
\hline 55720.00 & 7.00 & $5.69(0.02)(1.27,2.34)$ & 55768.00 & 55.00 & $5.21(0.02)(1.11,1.91)$ \\
\hline 55721.00 & 8.00 & $6.89(0.02)(1.54,2.82)$ & 55769.00 & 56.00 & $5.10(0.02)(1.09,1.87)$ \\
\hline 55722.00 & 9.00 & $8.09(0.02)(1.81,3.31)$ & 55770.00 & 57.00 & $4.99(0.02)(1.07,1.83)$ \\
\hline 55723.00 & 10.00 & $9.30(0.03)(2.07,3.79)$ & 55771.00 & 58.00 & $4.89(0.02)(1.05,1.79)$ \\
\hline 55724.00 & 11.00 & $10.44(0.03)(2.33,4.24)$ & 55772.00 & 59.00 & $4.80(0.02)(1.03,1.76)$ \\
\hline 55725.00 & 12.00 & $11.50(0.03)(2.56,4.65)$ & 55773.00 & 60.00 & $4.70(0.02)(1.01,1.73)$ \\
\hline 55726.00 & 13.00 & $12.45(0.03)(2.77,5.02)$ & 55774.00 & 61.00 & $4.61(0.02)(0.99,1.69)$ \\
\hline 55727.00 & 14.00 & $13.31(0.03)(2.95,5.35)$ & 55775.00 & 62.00 & $4.53(0.02)(0.97,1.66)$ \\
\hline 55728.00 & 15.00 & $14.07(0.04)(3.12,5.64)$ & 55776.00 & 63.00 & $4.44(0.02)(0.95,1.63)$ \\
\hline 55729.00 & 16.00 & $14.75(0.04)(3.27,5.90)$ & 55777.00 & 64.00 & $4.36(0.02)(0.93,1.60)$ \\
\hline 55730.00 & 17.00 & $15.38(0.05)(3.40,6.13)$ & 55778.00 & 65.00 & $4.27(0.02)(0.92,1.57)$ \\
\hline 55731.00 & 18.00 & $15.93(0.05)(3.52,6.34)$ & 55779.00 & 66.00 & $4.19(0.02)(0.90,1.54)$ \\
\hline 55732.00 & 19.00 & $16.36(0.05)(3.61,6.50)$ & 55780.00 & 67.00 & $4.11(0.02)(0.88,1.52)$ \\
\hline 55733.00 & 20.00 & $16.64(0.05)(3.67,6.60)$ & 55781.00 & 68.00 & $4.04(0.02)(0.87,1.49)$ \\
\hline 55734.00 & 21.00 & $16.72(0.05)(3.68,6.61)$ & 55782.00 & 69.00 & $3.96(0.02)(0.85,1.46)$ \\
\hline 55735.00 & 22.00 & $16.56(0.07)(3.64,6.51)$ & 55783.00 & 70.00 & $3.88(0.02)(0.83,1.43)$ \\
\hline 55736.00 & 23.00 & $16.16(0.06)(3.55,6.32)$ & 55784.00 & 71.00 & $3.80(0.02)(0.81,1.40)$ \\
\hline 55737.00 & 24.00 & $15.56(0.06)(3.41,6.05)$ & 55785.00 & 72.00 & $3.72(0.02)(0.80,1.38)$ \\
\hline 55738.00 & 25.00 & $14.83(0.05)(3.24,5.72)$ & 55786.00 & 73.00 & $3.65(0.02)(0.78,1.35)$ \\
\hline 55739.00 & 26.00 & $14.03(0.04)(3.05,5.37)$ & 55787.00 & 74.00 & $3.58(0.02)(0.77,1.33)$ \\
\hline 55740.00 & 27.00 & $13.20(0.04)(2.86,5.02)$ & 55788.00 & 75.00 & $3.51(0.02)(0.75,1.30)$ \\
\hline 55741.00 & 28.00 & $12.40(0.03)(2.68,4.69)$ & 55789.00 & 76.00 & $3.44(0.01)(0.74,1.28)$ \\
\hline 55742.00 & 29.00 & $11.67(0.04)(2.52,4.39)$ & 55790.00 & 77.00 & $3.38(0.01)(0.73,1.25)$ \\
\hline 55743.00 & 30.00 & $11.04(0.03)(2.38,4.13)$ & 55791.00 & 78.00 & $3.31(0.01)(0.71,1.23)$ \\
\hline 55744.00 & 31.00 & $10.46(0.03)(2.25,3.89)$ & 55792.00 & 79.00 & $3.25(0.01)(0.70,1.21)$ \\
\hline 55745.00 & 32.00 & $9.97(0.03)(2.14,3.70)$ & 55793.00 & 80.00 & $3.18(0.01)(0.68,1.18)$ \\
\hline 55746.00 & 33.00 & $9.54(0.03)(2.05,3.53)$ & 55794.00 & 81.00 & $3.12(0.01)(0.67,1.16)$ \\
\hline 55747.00 & 34.00 & 9.15 (0.03) (1.96,3.38) & 55795.00 & 82.00 & $3.06(0.01)(0.66,1.14)$ \\
\hline 55748.00 & 35.00 & $8.80(0.03)(1.89,3.24)$ & 55796.00 & 83.00 & $3.00(0.01)(0.65,1.12)$ \\
\hline 55749.00 & 36.00 & 8.49 (0.03) (1.82,3.12) & 55797.00 & 84.00 & $2.94(0.01)(0.63,1.10)$ \\
\hline 55750.00 & 37.00 & $8.21(0.03)(1.76,3.01)$ & 55798.00 & 85.00 & $2.88(0.01)(0.62,1.08)$ \\
\hline 55751.00 & 38.00 & $7.95(0.03)(1.70,2.91)$ & 55799.00 & 86.00 & $2.83(0.01)(0.61,1.06)$ \\
\hline 55752.00 & 39.00 & $7.71(0.02)(1.65,2.82)$ & 55800.00 & 87.00 & $2.77(0.01)(0.60,1.04)$ \\
\hline 55753.00 & 40.00 & $7.49(0.02)(1.60,2.74)$ & 55801.00 & 88.00 & $2.71(0.01)(0.58,1.02)$ \\
\hline 55754.00 & 41.00 & $7.27(0.02)(1.55,2.66)$ & 55802.00 & 89.00 & $2.66(0.01)(0.57,0.99)$ \\
\hline 55755.00 & 42.00 & $7.06(0.02)(1.51,2.58)$ & 55803.00 & 90.00 & $2.60(0.01)(0.56,0.97)$ \\
\hline 55756.00 & 43.00 & $6.86(0.02)(1.46,2.51)$ & 55804.00 & 91.00 & $2.55(0.01)(0.55,0.96)$ \\
\hline 55757.00 & 44.00 & $6.66(0.02)(1.42,2.43)$ & 55805.00 & 92.00 & $2.49(0.01)(0.54,0.94)$ \\
\hline 55758.00 & 45.00 & $6.50(0.02)(1.39,2.38)$ & 55806.00 & 93.00 & $2.44(0.01)(0.53,0.92)$ \\
\hline 55759.00 & 46.00 & $6.35(0.02)(1.36,2.32)$ & 55807.00 & 94.00 & $2.39(0.01)(0.52,0.90)$ \\
\hline 55760.00 & 47.00 & $6.20(0.02)(1.32,2.27)$ & 55808.00 & 95.00 & $2.34(0.01)(0.51,0.88)$ \\
\hline 55761.00 & 48.00 & $6.06(0.02)(1.29,2.21)$ & 55809.00 & 96.00 & $2.29(0.01)(0.50,0.86)$ \\
\hline 55762.00 & 49.00 & $5.93(0.02)(1.27,2.17)$ & 55810.00 & 97.00 & $2.25(0.01)(0.49,0.85)$ \\
\hline 55763.00 & 50.00 & $5.80(0.03)(1.24,2.12)$ & 55811.00 & 98.00 & $2.20(0.01)(0.48,0.83)$ \\
\hline 55764.00 & 51.00 & $5.68(0.02)(1.21,2.08)$ & 55812.00 & 99.00 & $2.16(0.01)(0.47,0.81)$ \\
\hline
\end{tabular}

Notes. Random errors are given in the first parentheses and systematic lower and upper errors (arising from the distance and extinction) respectively in the second parentheses. 


\section{Appendix A: Photometric calibration}

The optical photometry was tied to the Johnson-Cousins (JC) and Sloan Digital Sky Survey (SDSS) systems. The NIR photometry was tied to the 2 Micron All Sky Survey (2MASS) system. Table A.1 lists the filters used at each instrument and the mapping of these to the standard systems. Note that we have used JC-like $U B V R I$ filters and SDSS-like $g z$ filters at NOT whereas we have used JC-like $B V$ filters and SDSS-like ugriz filters at LT and FTN. The JC-like URI and SDSS-like uri photometry were then tied to both the JC and SDSS systems to produce full sets of JC and SDSS photometry. The Swift photometry was tied to the natural (photon count based) Vega system although the Swift $U B V$ photometry was also tied to the JC system for comparison. The Spitzer photometry was tied to the natural (energy flux based) Vega system.

\section{A.1. Calibration method}

The SN photometry was calibrated using reference stars within the SN field. These reference stars, in turn, were calibrated using standard fields. The calibration was performed using the SNE pipeline. To calibrate the SN photometry we fitted transformation equations of the type $m_{i}^{\text {sys }}=m_{i}^{\text {ins }}+C_{i, j k}\left(m_{j}^{\text {sys }}-m_{k}^{\text {sys }}\right)+Z_{i}$, where $m_{i}^{\text {sys }}, m_{i}^{\text {ins }}$ and $Z_{i}$ are the system and instrumental magnitudes and the zeropoint for band $i$ respectively and $C_{i, j k}$ is the colour-term coefficient for band $i$ using the colour $j k$. The magnitudes of the SN was evaluated both using these transformation equations and by the use of S-corrections. In the latter case the linear colour-terms are replaced by the S-corrections as determined from the SN spectra and the filter response functions of the natural systems of the instruments and the standard systems. We will discuss S-corrections in Appendix A.5 and compare the results from the two methods. In the end we have decided to use the S-corrected photometry for all bands except the JC $U$ and SDSS $u$ bands. To calibrate the reference star photometry we fitted transformation equations of the type $m_{i}^{\text {sys }}=m_{i}^{\text {ins }}+C_{i, j k}\left(m_{j}^{\text {sys }}-m_{k}^{\text {sys }}\right)+Z_{i}+e_{i} X_{i}$, where $X_{i}$ and $e_{i}$ are the airmass and the extinction coefficient for band $i$ respectively. The magnitudes of the reference stars were evaluated using these transformation equations and averaged using a magnitude error limit ( $0.05 \mathrm{mag})$ and mild $(3 \sigma)$ rejection. Both measurement errors and calibration errors in the fitted quantities were propagated using standard methods. The errors in the reference star magnitudes were calculated as the standard deviation of all measurements corrected for the degrees of freedom.

The coefficients of the linear colour terms $\left(C_{i, j k}\right)$ used to transform from the natural system of the instruments to the JC and SDSS standard systems were determined separately. For each instrument, system and band we determined the coefficient by least-square fitting of a common value to a large number of observations. For the NOT and the LT we also fitted the coefficients of a cross-term between colour and airmass for $U$ and $B$ to correct for the change in the filter response functions due to the variation of the extinction with airmass. However, given the colour and airmass range spanned by our observations, the correction turned out to be at the few-percent level and we decided to drop it. Because of the lower precision in the 2MASS catalogue as compared to the Landolt and SDSS catalogues we could not achieve the desired precision in measured 2MASS colourterm coefficients. Therefore we have used synthetic colour-term coefficients computed for a blackbody SED using the NIR filter response functions described in Sect. A.5. The measured JC and SDSS and synthetic 2MASS colour-term coefficients determined for each instrument are listed in Tables A.2-A.4.
Table A.1. Mapping of natural systems to standard systems.

\begin{tabular}{|c|c|c|}
\hline Telescope (Instrument) & Natural system & Standard system \\
\hline NOT (ALFOSC) & Bessel $U \# 7$ & JC U, SDSS $u$ \\
\hline NOT (ALFOSC) & Bessel $B \# 74$ & $\mathrm{JC} B$ \\
\hline NOT (ALFOSC) & Bessel $V \# 75$ & $\mathrm{JC} V$ \\
\hline NOT (ALFOSC) & Bessel $R \# 76$ & $\mathrm{JC} R, \operatorname{SDSS} r$ \\
\hline NOT (ALFOSC) & Interference i \#12 & $\mathrm{JC} I, \operatorname{SDSS} i$ \\
\hline NOT (ALFOSC) & SDSS $g \# 120$ & SDSS $g$ \\
\hline NOT (ALFOSC) & $\operatorname{SDSS} z \# 112$ & $\operatorname{SDSS} z$ \\
\hline LT (RATCam) & Sloan $u^{\prime}$ & $\operatorname{SDSS} u, \mathrm{JC} U$ \\
\hline LT (RATCam) & Sloan $g^{\prime}$ & SDSS $g$ \\
\hline LT (RATCam) & Sloan $r^{\prime}$ & SDSS $r$, JC $R$ \\
\hline LT (RATCam) & Sloan $i^{\prime}$ & SDSS $i, \mathrm{JC} I$ \\
\hline LT (RATCam) & Sloan $z^{\prime}$ & SDSS $z$ \\
\hline LT (RATCam) & Bessel $B$ & $\mathrm{JC} B$ \\
\hline LT (RATCam) & Bessel $V$ & $\mathrm{JC} V$ \\
\hline CA (CAFOS) & Johnson $U$ 370/47b & $\mathrm{JC} U$ \\
\hline CA (CAFOS) & Johnson $B 451 / 73$ & $\mathrm{JC} B$ \\
\hline CA (CAFOS) & Johnson $V$ 534/97b & JC $V$ \\
\hline CA (CAFOS) & Cousins $R 641 / 158$ & $\mathrm{JC} R$ \\
\hline CA (CAFOS) & Johnson $I 850 / 150 \mathrm{~b}$ & JC I \\
\hline ASIAGO (AFOSC) & Bessel $U$ & $\mathrm{JC} U$ \\
\hline ASIAGO (AFOSC) & Bessel $B$ & $\mathrm{JC} B$ \\
\hline ASIAGO (AFOSC) & Bessel $V$ & $\mathrm{JC} V$ \\
\hline ASIAGO (AFOSC) & Bessel $R$ & $\mathrm{JC} R$ \\
\hline ASIAGO (AFOSC) & Gunn $i$ & JC I \\
\hline ASIAGO (SCHMIDT) & $B$ & $\mathrm{JC} B$ \\
\hline ASIAGO (SCHMIDT) & V & JC $V$ \\
\hline ASIAGO (SCHMIDT) & $R$ & $\mathrm{JC} R$ \\
\hline ASIAGO (SCHMIDT) & $I$ & JC $I$ \\
\hline FTN (FSO2) & $\operatorname{SDSS} G$ & SDSS $g$ \\
\hline FTN (FSO2) & SDSS $R$ & SDSS $r$, JC $R$ \\
\hline FTN (FS02) & SDSS $I$ & SDSS $i$, JC I \\
\hline FTN (FS02) & Pann Starrs $Z$ & $\operatorname{SDSS} z$ \\
\hline MONTSEC (CCD) & $B$ & $\mathrm{JC} B$ \\
\hline MONTSEC (CCD) & $V$ & $\mathrm{JC} V$ \\
\hline MONTSEC (CCD) & $R$ & $\mathrm{JC} R$ \\
\hline MONTSEC (CCD) & $I$ & JC $I$ \\
\hline TNG (LRS) & Johnson $U$ & $\mathrm{JC} U$ \\
\hline TNG (LRS) & Johnson $B$ & $\mathrm{JC} B$ \\
\hline TNG (LRS) & Johnson $V$ & $\mathrm{JC} V$ \\
\hline TNG (LRS) & Cousins $R$ & $\mathrm{JC} R$ \\
\hline TNG (LRS) & Cousins $I$ & JC $I$ \\
\hline TNG (NICS) & $J$ & 2MASS $J$ \\
\hline TNG (NICS) & $H$ & 2MASS $H$ \\
\hline TNG (NICS) & $K$ & 2MASS $K$ \\
\hline TCS (CAIN) & $J$ & 2MASS $J$ \\
\hline TCS (CAIN) & $H$ & 2MASS $H$ \\
\hline TCS (CAIN) & Kshort & 2MASS $K$ \\
\hline WHT (LIRIS) & $j$ & 2MASS $J$ \\
\hline WHT (LIRIS) & $h$ & 2MASS $H$ \\
\hline WHT (LIRIS) & $k \mathrm{~s}$ & 2MASS $K$ \\
\hline NOT (NOTCAM) & $J$ & 2MASS $J$ \\
\hline NOT (NOTCAM) & $H$ & 2MASS $H$ \\
\hline NOT (NOTCAM) & $K \mathrm{~s}$ & 2MASS $K$ \\
\hline CA (O2000) & $J$ & 2MASS $J$ \\
\hline $\mathrm{CA}(\mathrm{O} 2000)$ & $H$ & 2MASS $H$ \\
\hline $\mathrm{CA}(\mathrm{O} 2000)$ & $K \mathrm{~S}$ & 2MASS $K$ \\
\hline LBT (LUCIFER) & $J$ & 2MASS $J$ \\
\hline LBT (LUCIFER) & $H$ & 2MASS $H$ \\
\hline LBT (LUCIFER) & $K \mathrm{~s}$ & 2MASS $K$ \\
\hline
\end{tabular}

\section{A.2. JC calibration}

The optical photometry was tied to the JC system using the reference stars presented in Pastorello et al. (2009, hereafter P09) as well as a number of additional fainter stars close to the SN. 
Table A.2. JC $U B V R I$ colour-term coefficients for all telescope/instrument combinations.

\begin{tabular}{lcccccccc}
\hline \hline $\mathrm{CT}$ & NOT (ALFOSC) & LT (RATCam) & FTN (FS02) & CA-2.2 m (CAFOS) & AS-Schmidt (SBIG) & AS-1.82 m (AFOSC) & TNG (LRS) & TJO (MEIA) \\
\hline $\mathrm{C}_{U, U B}$ & $0.127(0.005)$ & $0.018(0.006)$ & $\ldots$ & $0.186(0.025)$ & $\ldots$ & $0.179(0.048)$ & $0.181(0.020)$ & $\ldots$ \\
$\mathrm{C}_{U, U R}$ & $\ldots$ & $0.009(0.003)$ & $\ldots$ & $0.089(0.012)$ & $\ldots$ & $\ldots$ & $\ldots$ & $\ldots$ \\
$\mathrm{C}_{U, U V}$ & $\ldots$ & $\ldots$ & $\ldots$ & $\ldots$ & $\ldots$ & $0.106(0.028)$ & $0.107(0.011)$ & $\ldots$ \\
$\mathrm{C}_{B, B V}$ & $0.039(0.005)$ & $0.059(0.005)$ & $\ldots$ & $0.135(0.010)$ & $0.133(0.041)$ & $0.035(0.014)$ & $0.071(0.004)$ & $0.237(0.016)$ \\
$\mathrm{C}_{B, U B}$ & $\ldots$ & $0.039(0.004)$ & $\ldots$ & $0.095(0.007)$ & $\ldots$ & $0.024(0.010)$ & $0.050(0.006)$ & $\ldots$ \\
$\mathrm{C}_{B, B R}$ & $\ldots$ & $\ldots$ & $\ldots$ & $\ldots$ & $0.086(0.026)$ & $\ldots$ & $\ldots$ & $\ldots$ \\
$\mathrm{C}_{B, B I}$ & $\ldots$ & $\ldots$ & $\ldots$ & $\ldots$ & $0.065(0.020)$ & $\ldots$ & $\ldots$ \\
$\mathrm{C}_{V, B V}$ & $-0.048(0.007)$ & $-0.058(0.006)$ & $\ldots$ & $-0.036(0.009)$ & $-0.075(0.043)$ & $0.034(0.016)$ & $-0.053(0.009)$ & $-0.038(0.008)$ \\
$\mathrm{C}_{V, V R}$ & $\ldots$ & $-0.103(0.009)$ & $\ldots$ & $-0.060(0.016)$ & $-0.083(0.069)$ & $0.064(0.029)$ & $-0.096(0.018)$ & $-0.072(0.014)$ \\
$\mathrm{C}_{R, V R}$ & $-0.064(0.010)$ & $-0.180(0.006)$ & $\ldots$ & $0.051(0.014)$ & $-0.054(0.050)$ & $0.043(0.032)$ & $-0.031(0.015)$ & $-0.061(0.020)$ \\
$\mathrm{C}_{R, R I}$ & $\ldots$ & $-0.183(0.011)$ & $-0.324(0.014)$ & $0.043(0.011)$ & $-0.050(0.036)$ & $0.048(0.032)$ & $-0.029(0.015)$ & $-0.066(0.020)$ \\
$\mathrm{C}_{I, V I}$ & $-0.021(0.004)$ & $-0.085(0.006)$ & $\ldots$ & $0.072(0.020)$ & $0.024(0.018)$ & $-0.012(0.015)$ & $0.061(0.018)$ & $0.041(0.010)$ \\
$\mathrm{C}_{I, R I}$ & $\ldots$ & $-0.168(0.012)$ & $-0.231(0.019)$ & $0.128(0.038)$ & $0.039(0.031)$ & $-0.021(0.030)$ & $0.122(0.034)$ & $0.082(0.022)$ \\
$\mathrm{C}_{I, B I}$ & $\ldots$ & $\ldots$ & $\ldots$ & $\ldots$ & $0.013(0.011)$ & $\ldots$ & $\ldots$ \\
\hline
\end{tabular}

Notes. NOT colour-term coefficients provided by V. Stanishev (Priv. Comm.). Errors are given in parentheses.

Table A.3. SDSS ugriz colour-term coefficients for all telescope/instrument combinations.

\begin{tabular}{lccc}
\hline \hline $\mathrm{CT}$ & NOT (ALFOSC) & LT (RATCam) & FTN (FS02) \\
\hline $\mathrm{C}_{u, u g}$ & $0.043(0.027)$ & $0.032(0.024)$ & $\ldots$ \\
$\mathrm{C}_{u, u r}$ & $0.029(0.018)$ & $0.021(0.015)$ & $\ldots$ \\
$\mathrm{C}_{g, g r}$ & $0.009(0.011)$ & $0.102(0.012)$ & $0.200(0.038)$ \\
$\mathrm{C}_{g, u g}$ & $0.007(0.008)$ & $0.056(0.010)$ & $\ldots$ \\
$\mathrm{C}_{r, g r}$ & $0.077(0.019)$ & $0.023(0.016)$ & $0.032(0.014)$ \\
$\mathrm{C}_{r, r i}$ & $0.162(0.016)$ & $0.057(0.020)$ & $0.045(0.014)$ \\
$\mathrm{C}_{i, r i}$ & $0.185(0.027)$ & $0.094(0.009)$ & $0.045(0.011)$ \\
$\mathrm{C}_{i, i z}$ & $0.310(0.038)$ & $0.159(0.015)$ & $0.082(0.018)$ \\
$\mathrm{C}_{z, i z}$ & $-0.094(0.046)$ & $-0.081(0.065)$ & $-0.119(0.023)$ \\
$\mathrm{C}_{z, r z}$ & $-0.027(0.019)$ & $-0.024(0.028)$ & $\ldots$ \\
\hline
\end{tabular}

Notes. Errors are given in parentheses.

In the following the reference stars from P09 will be abbreviated as P09-N and those added in this paper as E13-N. Those reference stars, in turn, have been tied to the JC system using standard fields from Landolt (1983, 1992). Taking advantage of the large number of standard star observations obtained with the LT we have re-measured the magnitudes of the P09 reference stars within the LT field of view (FOV). The mean and root mean square (RMS) of the difference was at the few-percent level for the $B, V, R$ and $I$ bands and at the 10-percent level for the $U$ band except for P09-3 which differed considerably. We have also remeasured the magnitudes of the Landolt standard stars and the mean and RMS of the difference was at the few-percent level in all bands. This shows that, in spite of the SDSS-like nature of the LT $u, r$ and $i$ filters, the natural LT photometry transform to the JC system with good precision. In the end we chose to keep the P09 magnitudes, except for P09-3, and use the LT observations as confirmation. The magnitudes of the additional reference stars and P09-3 were determined using the remaining P09 reference stars and large number of deep, high quality NOT images of the SN field. The coordinates and magnitudes of the JC reference stars are listed Table A.5 and their positions marked in Fig. A.1.

\section{A.3. SDSS calibration}

The optical photometry was tied to the SDSS system using the subset of the reference stars within the LT FOV. Those reference stars, in turn, have been tied to the SDSS system using fields

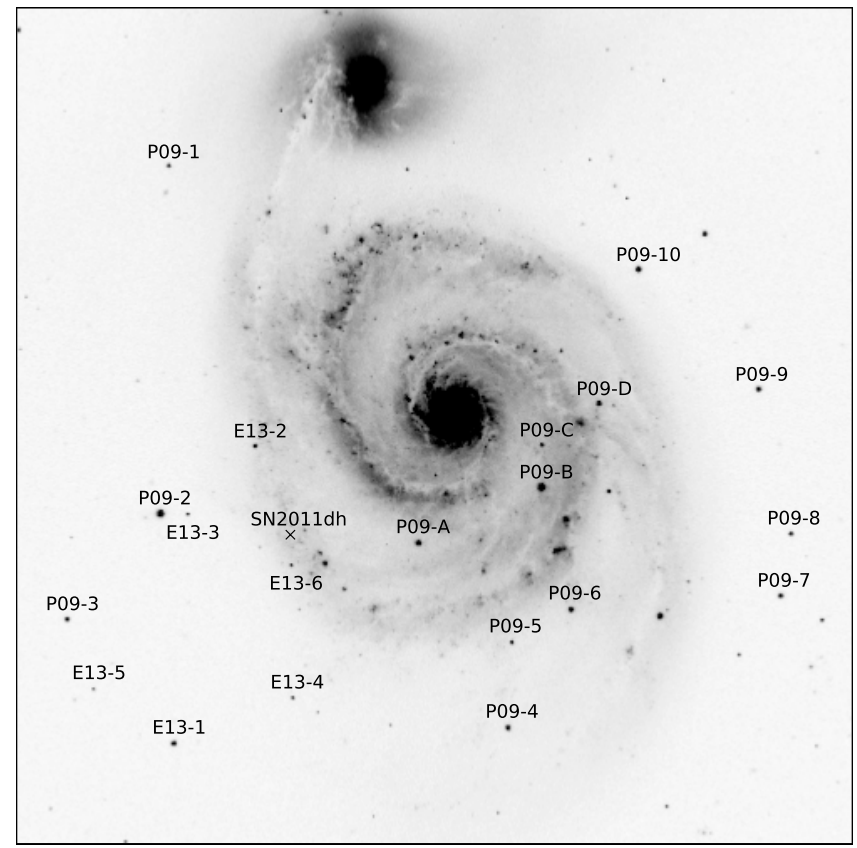

Fig. A.1. Reference stars used for calibration of the optical photometry marked on a SDSS $r$ band image.

covered by the SDSS DR8 catalogue (Aihara et al. 2011). The calibration was not straightforward as the SN field and a number of the LT standard fields were not well covered by the catalogue and many of the brighter stars in the fields covered by the catalogue were marked as saturated in the $g, r$ and $i$ bands. The procedure used to calibrate the reference star magnitudes was as follows. First we re-measured the $g, r$ and $i$ magnitudes for all stars marked as saturated using the remaining stars in the LT standard fields covered by the catalogue. We then measured the magnitudes for the stars in the fields not covered by the catalogue and finally we measured the reference star magnitudes using all the LT standard field observations. For reference, our measured SDSS magnitudes for the LT standard fields are listed in Table A.6. Magnitudes of stars covered by the catalogue and not marked as saturated were adopted from the catalogue. The coordinates and the magnitudes of the SDSS reference stars are listed in Table A.7 and their positions marked in Fig. A.1. The magnitudes of P09-3, E13-1 and E13-5 were adopted from the 
A\&A 562, A17 (2014)

Table A.4. Synthetic 2MASS $J H K$ colour-term coefficients for all telescope/instrument combinations.

\begin{tabular}{lcccccc}
\hline \hline $\mathrm{CT}$ & TNG (NICS) & NOT (NOTCAM) & TCS (CAIN) & LBT (LUCIFER) & WHT (LIRIS) & CA-3.5 m (O2000) \\
\hline $\mathrm{C}_{J, J H}$ & 0.028 & 0.039 & 0.026 & -0.032 & 0.056 & -0.092 \\
$\mathrm{C}_{J, J K}$ & 0.017 & 0.024 & 0.016 & -0.020 & 0.034 & -0.055 \\
$\mathrm{C}_{H, J H}$ & -0.006 & -0.008 & 0.051 & 0.021 & -0.011 & 0.019 \\
$\mathrm{C}_{H, H K}$ & -0.008 & -0.011 & 0.077 & 0.032 & -0.016 & 0.028 \\
$\mathrm{C}_{K, J K}$ & 0.025 & -0.005 & 0.027 & 0.001 & -0.003 & -0.007 \\
$\mathrm{C}_{K, H K}$ & 0.062 & -0.012 & 0.067 & 0.001 & -0.007 & -0.018 \\
\hline
\end{tabular}

Table A.5. JC UBVRI magnitudes of local reference stars used to calibrate the photometry.

\begin{tabular}{lccccccc}
\hline \hline ID & $\begin{array}{c}\text { RA } \\
(\mathrm{h} \mathrm{m} \mathrm{s})\end{array}$ & $\begin{array}{c}\text { Dec } \\
\left({ }^{\circ}{ }^{\prime \prime}\right)\end{array}$ & $\begin{array}{c}U \\
(\mathrm{mag})\end{array}$ & $\begin{array}{c}B \\
(\mathrm{mag})\end{array}$ & $\begin{array}{c}V \\
(\mathrm{mag})\end{array}$ & $\begin{array}{c}R \\
(\mathrm{mag})\end{array}$ & $\begin{array}{c}I \\
(\mathrm{mag})\end{array}$ \\
\hline P09-1 & 133014.2 & +471455 & $\ldots$ & $17.01(0.18)$ & $16.27(0.03)$ & $15.81(0.08)$ & $15.44(0.06)$ \\
P09-2 & 133014.9 & +471027 & $14.62(0.04)$ & $14.32(0.02)$ & $13.60(0.01)$ & $13.19(0.01)$ & $12.81(0.01)$ \\
P09-3 & 133021.9 & +470905 & $16.63(0.01)$ & $16.55(0.01)$ & $15.92(0.01)$ & $15.48(0.01)$ & $15.10(0.01)$ \\
P09-4 & 132948.5 & +470742 & $16.15(0.05)$ & $16.23(0.02)$ & $15.66(0.02)$ & $15.23(0.01)$ & $14.91(0.03)$ \\
P09-5 & 132948.2 & +470848 & $18.88(0.07)$ & $18.25(0.07)$ & $17.36(0.01)$ & $16.75(0.02)$ & $16.28(0.01)$ \\
P09-6 & 132943.7 & +470914 & $15.51(0.02)$ & $15.85(0.01)$ & $15.39(0.02)$ & $15.05(0.01)$ & $14.74(0.01)$ \\
P09-7 & 132927.9 & +470923 & $17.13(0.19)$ & $16.73(0.01)$ & $16.06(0.02)$ & $15.54(0.01)$ & $15.10(0.05)$ \\
P09-8 & 132927.1 & +471012 & $19.76(0.02)$ & $18.72(0.05)$ & $17.41(0.04)$ & $15.82(0.03)$ & $14.38(0.03)$ \\
P09-9 & 132929.5 & +471203 & $18.01(0.23)$ & $16.66(0.03)$ & $15.68(0.01)$ & $14.97(0.01)$ & $14.36(0.04)$ \\
P09-10 & 132938.6 & +471336 & $16.74(0.04)$ & $16.20(0.03)$ & $15.29(0.01)$ & $14.75(0.02)$ & $14.20(0.02)$ \\
P09-A & 132955.3 & +471005 & $17.77(0.03)$ & $16.34(0.01)$ & $15.11(0.01)$ & $14.33(0.01)$ & $13.68(0.01)$ \\
P09-B & 132945.9 & +471047 & $14.12(0.02)$ & $14.01(0.01)$ & $13.43(0.01)$ & $13.06(0.01)$ & $12.73(0.01)$ \\
P09-C & 132945.9 & +471120 & $17.19(0.04)$ & $17.15(0.02)$ & $16.67(0.01)$ & $16.29(0.01)$ & $15.94(0.01)$ \\
P09-D & 132941.6 & +471152 & $15.76(0.03)$ & $15.77(0.01)$ & $15.24(0.01)$ & $14.85(0.01)$ & $14.52(0.01)$ \\
E13-1 & 133013.8 & +470730 & $17.35(0.01)$ & $16.59(0.01)$ & $15.70(0.01)$ & $15.12(0.01)$ & $14.67(0.01)$ \\
E13-2 & 133007.6 & +471119 & $17.76(0.01)$ & $17.26(0.01)$ & $16.48(0.01)$ & $16.02(0.01)$ & $15.63(0.01)$ \\
E13-3 & 133012.8 & +471027 & $18.94(0.02)$ & $18.58(0.01)$ & $17.82(0.01)$ & $17.29(0.01)$ & $16.84(0.01)$ \\
E13-4 & 133004.8 & +470805 & $20.57(0.03)$ & $19.46(0.01)$ & $17.99(0.01)$ & $16.98(0.01)$ & $15.61(0.01)$ \\
E13-5 & 133019.9 & +470812 & $19.79(0.03)$ & $19.17(0.01)$ & $18.34(0.01)$ & $17.76(0.01)$ & $17.31(0.01)$ \\
E13-6 & 133004.9 & +470947 & $20.98(0.06)$ & $19.61(0.01)$ & $18.20(0.01)$ & $17.28(0.01)$ & $16.41(0.01)$ \\
\hline
\end{tabular}

Notes. Errors are given in parentheses.

Table A.6. SDSS ugriz magnitudes for the standard fields PG 0231+051, PG 1047+003, PG 1525-071, PG 2331+046 and Mark-A.

\begin{tabular}{|c|c|c|c|c|c|c|c|}
\hline ID & $\begin{array}{c}\text { RA } \\
(\mathrm{h} \mathrm{m} \mathrm{s})\end{array}$ & $\begin{array}{c}\text { Dec } \\
\left({ }^{\circ}, \prime\right)\end{array}$ & $\begin{array}{c}u \\
(\mathrm{mag})\end{array}$ & $\begin{array}{c}g \\
(\mathrm{mag})\end{array}$ & $\begin{array}{c}r \\
(\mathrm{mag})\end{array}$ & $\begin{array}{c}i \\
(\mathrm{mag})\end{array}$ & $\begin{array}{c}z \\
\text { (mag) }\end{array}$ \\
\hline PG $0231+051 \mathrm{E}$ & 023328.8 & +051948 & $15.56(0.01)$ & & & & $13.41(0.02)$ \\
\hline PG $0231+051 \mathrm{D}$ & 023334.0 & +051930 & $17.00(0.02)$ & $14.59(0.02)$ & $13.62(0.01)$ & $13.24(0.02)$ & $13.04(0.02)$ \\
\hline PG $0231+051 \mathrm{~A}$ & 023340.0 & +051740 & $14.63(0.02)$ & $13.07(0.02)$ & $12.55(0.01)$ & $12.39(0.01)$ & $12.31(0.02)$ \\
\hline PG $0231+051$ & 023341.3 & +051843 & $15.36(0.01)$ & $15.80(0.02)$ & $16.30(0.02)$ & $16.61(0.02)$ & $16.96(0.02)$ \\
\hline PG $0231+051 B$ & 023345.5 & +051733 & $18.28(0.02)$ & $15.54(0.02)$ & $14.16(0.01)$ & $13.36(0.01)$ & $12.93(0.02)$ \\
\hline PG $0231+051 \mathrm{C}$ & 023348.1 & +052026 & $15.33(0.01)$ & $13.97(0.02)$ & $13.49(0.01)$ & $13.33(0.01)$ & $13.29(0.02)$ \\
\hline PG 1047+003 & 105002.8 & -000036 & & $13.23(0.02)$ & $13.68(0.01)$ & 14.06( & $14.40(0.03)$ \\
\hline PG $1047+003 \mathrm{~A}$ & 105005.6 & -000 & $15.22(0.01)$ & $36(0$ & 2( & 1) & 03) \\
\hline $47+003 B$ & 105007.9 & -000204 & $16.45(0.01)$ & $15.02(0.02)$ & $14.54(0$ & 14.45 & 03) \\
\hline PG $1047+003 C$ & 105013.6 & -000031 & $14.04(0.01)$ & $12.72(0.02)$ & $12.27(0.01)$ & 12.14 & $12.20(0.03)$ \\
\hline PG 1525-071 & 152811.5 & -071632 & $14.44(0.01)$ & $14.84(0.01)$ & $15.24(0.01)$ & $15.56(0.01)$ & $15.90(0.01)$ \\
\hline PG 1525-071D & 152812.0 & -071639 & $17.84(0.03)$ & $16.64(0.01)$ & $16.12(0.01)$ & $15.96(0.01)$ & $15.89(0.01)$ \\
\hline PG $1525-071 \mathrm{~A}$ & 152813.4 & -071601 & $15.38(0.01)$ & $13.88(0.01)$ & $13.29(0.01)$ & $13.09(0.01)$ & $13.01(0.01)$ \\
\hline PG 1525-071B & 152814.3 & -071613 & $18.01(0.04)$ & $16.73(0.01)$ & $16.17(0.01)$ & $15.98(0.01)$ & $15.88(0.01)$ \\
\hline PG 1525-071C & 152816.5 & -071430 & $16.57(0.02)$ & $14.10(0.01)$ & $13.20(0.01)$ & $12.90(0.01)$ & $12.74(0.01)$ \\
\hline PG $2331+055$ & 233344.4 & +054639 & $15.43(0.02)$ & $15.04(0.02)$ & $15.30(0.02)$ & $15.52(0.02)$ & $15.70(0.02)$ \\
\hline PG $2331+055 \mathrm{~A}$ & 233349.3 & +054652 & $14.85(0.02)$ & $13.36(0.01)$ & $12.85(0.01)$ & $12.65(0.01)$ & $12.56(0.02)$ \\
\hline PG $2331+055 B$ & 233351.1 & +054508 & $16.79(0.02)$ & $15.10(0.02)$ & $14.47(0.02)$ & $14.21(0.02)$ & $14.07(0.02)$ \\
\hline MarkA4 & 204353.5 & -104505 & $16.53(0.02)$ & $15.14(0.01)$ & $14.53(0.01)$ & $14.25(0.01)$ & $14.10(0.01)$ \\
\hline MarkA2 & 204354.9 & -104531 & $16.10(0.01)$ & $14.82(0.01)$ & $14.37(0.01)$ & $14.21(0.01)$ & $14.15(0.01)$ \\
\hline MarkA1 & 204358.4 & -104712 & $17.26(0.02)$ & $16.16(0.01)$ & $15.73(0.01)$ & $15.57(0.01)$ & $15.51(0.01)$ \\
\hline MarkA & 204359.2 & -104741 & $12.61(0.01)$ & $12.97(0.01)$ & $13.47(0.01)$ & $13.78(0.01)$ & $14.12(0.01)$ \\
\hline MarkA3 & 204403.8 & -104537 & $17.20(0.02)$ & $15.28(0.01)$ & $14.48(0.01)$ & $14.17(0.01)$ & $13.99(0.01)$ \\
\hline
\end{tabular}

Notes. Errors are given in parentheses. 
M. Ergon et al.: SN 2011dh - The first 100 days

Table A.7. SDSS ugriz magnitudes of local reference stars used to calibrate the photometry.

\begin{tabular}{lccccccc}
\hline \hline ID & $\begin{array}{c}\text { RA } \\
(\mathrm{h} \mathrm{m} \mathrm{s})\end{array}$ & $\begin{array}{c}\text { Dec } \\
\left({ }^{\circ}{ }^{\prime \prime}\right)\end{array}$ & $\begin{array}{c}u \\
(\mathrm{mag})\end{array}$ & $\begin{array}{c}g \\
(\mathrm{mag})\end{array}$ & $\begin{array}{c}r \\
(\mathrm{mag})\end{array}$ & $\begin{array}{c}i \\
(\mathrm{mag})\end{array}$ & $\begin{array}{c}z \\
(\mathrm{mag})\end{array}$ \\
\hline P09-1 & 133014.2 & +471455 & $17.99(0.02)$ & $16.64(0.02)$ & $16.09(0.01)$ & $15.86(0.02)$ & $15.77(0.02)$ \\
P09-2 & 133014.9 & +471027 & $15.59(0.01)$ & $13.96(0.02)$ & $13.42(0.01)$ & $13.31(0.02)$ & $13.21(0.02)$ \\
P09-3 & 133021.9 & +470905 & $\ldots$ & $16.15(0.02)$ & $15.69(0.02)$ & $15.52(0.01)$ & $15.39(0.02)$ \\
P09-4 & 132948.5 & +470742 & $17.07(0.02)$ & $15.90(0.01)$ & $15.45(0.01)$ & $15.30(0.01)$ & $15.25(0.01)$ \\
P09-5 & 132948.2 & +470848 & $19.33(0.17)$ & $17.84(0.02)$ & $17.00(0.01)$ & $16.71(0.01)$ & $16.53(0.02)$ \\
P09-6 & 132943.7 & +470914 & $16.41(0.01)$ & $15.55(0.01)$ & $15.24(0.01)$ & $15.13(0.01)$ & $15.10(0.01)$ \\
P09-7 & 132927.9 & +470923 & $\ldots$ & $\ldots$ & $\ldots$ & $\ldots$ & $\ldots$ \\
P09-8 & 132927.1 & +471012 & $\ldots$ & $\ldots$ & $\ldots$ & $\ldots$ & $\ldots$ \\
P09-9 & 132929.5 & +471203 & $\ldots$ & $\ldots$ & $\ldots$ & $\ldots$ & $\ldots$ \\
P09-10 & 132938.6 & +471336 & $\ldots$ & $\ldots$ & $\ldots$ & $\ldots$ & \\
P09-A & 132955.3 & +471005 & $18.52(0.03)$ & $15.86(0.01)$ & $14.65(0.01)$ & $14.22(0.01)$ & $13.99(0.01)$ \\
P09-B & 132945.9 & +471047 & $14.95(0.02)$ & $13.71(0.01)$ & $13.26(0.01)$ & $13.15(0.01)$ & $13.12(0.01)$ \\
P09-C & 132945.9 & +471120 & $17.88(0.11)$ & $16.88(0.01)$ & $16.48(0.01)$ & $16.38(0.01)$ & $16.33(0.05)$ \\
P09-D & 132941.6 & +471152 & $16.64(0.04)$ & $15.49(0.01)$ & $15.09(0.01)$ & $14.99(0.01)$ & $14.95(0.02)$ \\
E13-1 & 133013.8 & +470730 & $18.03(0.02)$ & $16.12(0.02)$ & $15.32(0.02)$ & $15.08(0.01)$ & $14.93(0.02)$ \\
E13-2 & 133007.6 & +471119 & $\ldots$ & $16.86(0.03)$ & $16.23(0.03)$ & $16.04(0.01)$ & $15.96(0.03)$ \\
E13-3 & 133012.8 & +471027 & $\ldots$ & $18.23(0.02)$ & $17.53(0.01)$ & $17.32(0.04)$ & $17.04(0.02)$ \\
E13-4 & 133004.8 & +470805 & $\ldots$ & $18.76(0.02)$ & $17.37(0.01)$ & $16.27(0.01)$ & $15.61(0.01)$ \\
E13-5 & 133019.9 & +470812 & $20.54(0.06)$ & $18.67(0.02)$ & $17.95(0.02)$ & $17.72(0.02)$ & $17.56(0.03)$ \\
E13-6 & 133004.9 & +470947 & $\ldots$ & $18.96(0.03)$ & $17.59(0.01)$ & $16.90(0.01)$ & $16.48(0.02)$ \\
\hline
\end{tabular}

Notes. Errors are given in parentheses.

catalogue. The mean and RMS of the difference between measured and catalogue magnitudes for stars with measured and catalogue errors less than 5 percent was less than 5 percent in all bands.

\section{A.4. 2MASS calibration}

The NIR photometry was tied to the 2MASS system using all stars within 7 arcmin distance from the SN with $J$ magnitude brighter than 18.0 detected in deep UKIRT imaging of the SN field. This includes the optical reference stars as well as $\sim 50$ additional stars, although for most observations the small FOV prevented use of more than about 10 of these. Those reference stars, in turn, have been tied to the 2MASS system using all stars from the 2MASS Point Source catalogue (Skrutskie et al. 2006) with $J$ magnitude error less than 0.05 mag within the $13.65 \times 13.65$ arc minute FOV. The coordinates and magnitudes for the 2MASS reference stars are listed in Table A.8. Magnitudes of stars covered by the catalogue and with errors less than 0.05 were adopted from the catalogue. The mean and RMS of the difference between measured and catalogue magnitudes for stars with measured and catalogue errors less than 5 percent was less than 5 percent in all bands.

\section{A.5. S-corrections}

From the above and the fact that a fair (usually 5-10) number of reference stars were used we conclude that the calibration of the JC, SDSS and 2MASS photometry, with the possible exception of the $U$ band, should be good to the few-percent level as long as a linear colour-term is sufficient to transform to the standard systems. This is known to work well for stars, but is not necessarily true for SNe. Photometry for well monitored SNe as 1987A and 1993J shows significant differences between different datasets and telescopes, in particular at late times, as might be expected by the increasingly line-dominated nature of the spectrum. A more elaborate method to transform from the natural system to the standard system is S-corrections (Stritzinger et al. 2002). Using this method we first transform the reference star magnitudes to the natural system using linear colour-terms and then transform the SN magnitudes to the standard system by replacing the linear colour-terms with S-corrections calculated as the difference of the synthetic magnitudes in the standard and natural systems. Note that this definition differs from the one by Stritzinger et al. (2002) but is the same as used by Taubenberger et al. (2011). Success of the method depends critically on the accuracy of the filter response functions and a well sampled spectral sequence.

For all telescopes we constructed optical filter response functions from filter and CCD data provided by the observatory or the manufacturer and extinction data for the site. Extinction data for Roque de los Muchachos at La Palma where NOT, LT, TNG and WHT are located were obtained from the Isaac Newton Group of Telescopes (ING), extinction data for Manua Kea where FTN is located were obtained from the Gemini Observatory and extinction data for Calar Alto and Mount Ekar were adopted from the QUBA pipeline. For TJO and the amateur telescopes we have assumed the same extinction as at Calar Alto. A typical telluric absorption profile, as determined from WHT and TNG spectroscopy, was also added to the filter response functions. We have assumed that the optics response functions vary slowly enough not to affect the S-corrections. To test this we constructed optics response functions for a number of telescopes from filter zeropoints, measured from standard star data or provided by the observatories. Except below $\sim 4000 \AA$ they vary slowly as expected and applying them to our data the S-corrections vary at the percent level or less so the assumption seems to be justified. Below $\sim 4000 \AA$ the optics response functions may vary rapidly and because of this and other difficulties in this wavelength region we have not applied S-corrections to the JC $U$ and SDSS $u$ bands. To test the constructed JC and SDSS filter response functions we compared synthetic colour-term coefficients derived from the STIS NGSL ${ }^{4}$

4 http://archive.stsci.edu/prepds/stisngsl/ 
A\&A 562, A17 (2014)

Table A.8. 2MASS $J H K$ magnitudes of local reference stars used to calibrate the photometry.

\begin{tabular}{|c|c|c|c|c|c|}
\hline ID & $\begin{array}{c}\mathrm{RA} \\
(\mathrm{h} \mathrm{m} \mathrm{s})\end{array}$ & $\begin{array}{c}\text { Dec } \\
\left(0^{\circ}, \prime \prime\right)\end{array}$ & $\begin{array}{c}J \\
(\mathrm{mag})\end{array}$ & $\begin{array}{c}H \\
\text { (mag) }\end{array}$ & $\begin{array}{c}K \\
(\mathrm{mag})\end{array}$ \\
\hline P09-1 & 133014.3 & +471455 & $14.87(0.01)$ & $14.43(0.01)$ & $14.36(0.01)$ \\
\hline P09-2 & 3014.8 & 4710 & 12.35 & 12.02 & 11.96 \\
\hline P09-3 & 3021.9 & +470905 & 01) & $.01)$ & 1400 \\
\hline P09-4 & 132948.5 & +470742 & $.01)$ & 14.0 & 1300 \\
\hline P09-5 & 132948.2 & +470848 & 15.52 & $.01)$ & 1) \\
\hline P09-6 & 132943.7 & +470913 & $28(0.01)$ & 13.99 & 13.93 \\
\hline P09-7 & 132927.8 & +470923 & $14.46(0.01)$ & $14.00(0.01)$ & 13.91 \\
\hline P09-8 & 132927.0 & +471010 & $12.90(0.01)$ & $12.38(0.01)$ & 12.12 \\
\hline P09-9 & 132929.4 & +471203 & $13.52(0.01)$ & $12.91(0.01)$ & $12.79(0.01)$ \\
\hline P09-10 & 132938.6 & +471335 & $13.46(0.01)$ & $12.93(0.01)$ & $12.79(0.01)$ \\
\hline P09-A & & +471004 & & & \\
\hline P09-B & 13294 & +471047 & & & \\
\hline P09-C & 13294 & +471120 & & & \\
\hline $\mathrm{DOO} D$ & 13294 & +471152 & & & \\
\hline & & & & & \\
\hline & & & & & \\
\hline & 13 & + & & & \\
\hline-4 & 1 & + & & & \\
\hline $\mathrm{E}$ & 1 & + & & & \\
\hline $\mathrm{E}$ & 1 & 7 & & & \\
\hline E13-7 & 1325 & + & & & \\
\hline E13-8 & 132 & +470 & & & \\
\hline-9 & 13293 & +471403 & & & \\
\hline & & & & & \\
\hline E & 1. & +4 & & & \\
\hline E13-12 & 132 & +47 & & & \\
\hline & & +47 & & & \\
\hline & & + & & & \\
\hline & & & & & \\
\hline & & & & & \\
\hline & & & & & \\
\hline & & & & & \\
\hline & 6 & + & & & \\
\hline & 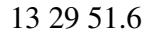 & 0 & & & \\
\hline E13-21 & 13295 & +4710 & & & \\
\hline E13-22 & 13295 & +4713 & & & \\
\hline & 2 & +471 & & & \\
\hline & 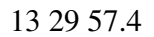 & 470 & & & \\
\hline & & & & & \\
\hline & 1 & +470 & & & \\
\hline E13-27 & 133 & +471 & & 17. & 5) \\
\hline & & +4707 & & & \\
\hline & & & & & \\
\hline & & & & & \\
\hline & & & & & \\
\hline & & + & & & \\
\hline & & & & & \\
\hline & & 0 & & & \\
\hline E & 0 & +470 & & & \\
\hline E & 3010.0 & +4714 & ) & $1^{\prime}$ & 17.21 \\
\hline & 4 & 705 & & 1) & 5.18 \\
\hline & 201 & +4711 & & & \\
\hline & 1 & 70 & & & \\
\hline & 301 & 71 & & & \\
\hline E &  & + & & 1) & \\
\hline $3-42$ & & +4706 & & & 5.99 \\
\hline E13-43 & 133026.7 & +470726 & 10.010. & $14.79(0.01)$ & $4.57(0.01)$ \\
\hline E13-44 & 133030.2 & +471234 & $16.34(0.01)$ & $15.70(0.01)$ & $5.44(0.01)$ \\
\hline-45 & 13 & +4712 & ) & 1) & $.06)$ \\
\hline E1 & & +470639 & & & \\
\hline-47 & 13 & +4714 & ) & 1. & J. \\
\hline & & +4713 & & 15.7 & 15.68 \\
\hline E13-49 & 133034.8 & +471229 & $15.08(0.01)$ & $14.47(0.01)$ & $14.26(0.01)$ \\
\hline
\end{tabular}

Notes. Errors are given in parentheses. 
Table A.8. continued.

\begin{tabular}{lccccc}
\hline \hline ID & $\begin{array}{c}\text { RA } \\
(\mathrm{h} \mathrm{m} \mathrm{s})\end{array}$ & $\begin{array}{c}\text { Dec } \\
\left({ }^{\prime} \prime^{\prime \prime}\right)\end{array}$ & $\begin{array}{c}J \\
(\mathrm{mag})\end{array}$ & $\begin{array}{c}H \\
(\mathrm{mag})\end{array}$ & $\begin{array}{c}K \\
(\mathrm{mag})\end{array}$ \\
\hline E13-50 & 133035.9 & +470719 & $17.98(0.03)$ & $17.63(0.04)$ & $17.50(0.01)$ \\
E13-51 & 133036.6 & +470917 & $15.42(0.01)$ & $14.80(0.01)$ & $14.58(0.01)$ \\
E13-52 & 133039.8 & +471119 & $17.96(0.01)$ & $17.43(0.04)$ & $17.21(0.04)$ \\
E13-53 & 133042.4 & +471011 & $14.71(0.01)$ & $14.03(0.01)$ & $13.93(0.01)$ \\
E13-54 & 133046.2 & +471004 & $16.58(0.01)$ & $15.96(0.01)$ & $15.72(0.03)$ \\
\hline
\end{tabular}

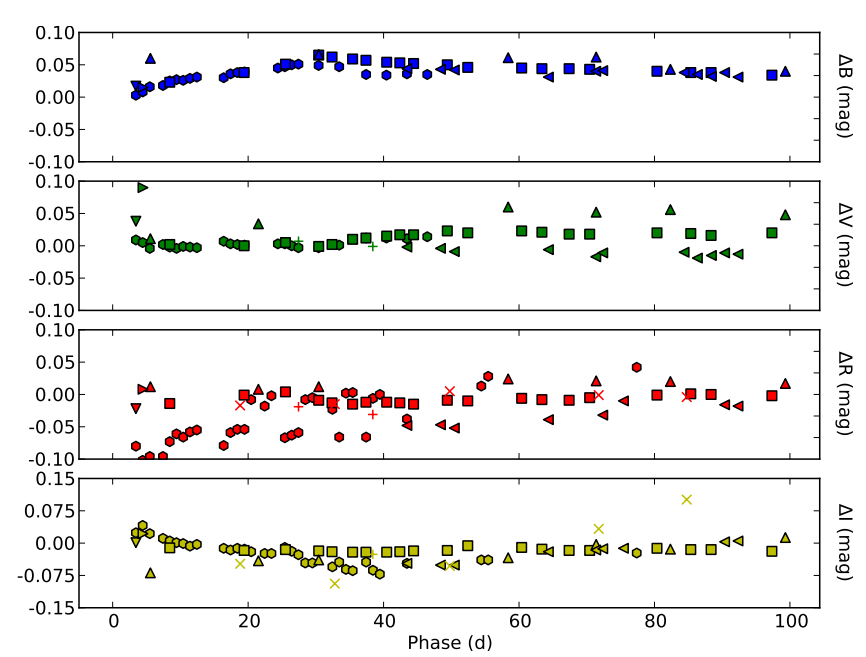

Fig. A.2. Difference between JC colour- and S-corrected photometry for NOT (squares), LT (circles), CA 2.2 m (upward triangles), TNG (downward triangles), AS $1.82 \mathrm{~m}$ (rightward triangles), AS Schmidt (leftward triangles), TJO (pluses) and FTN (crosses).

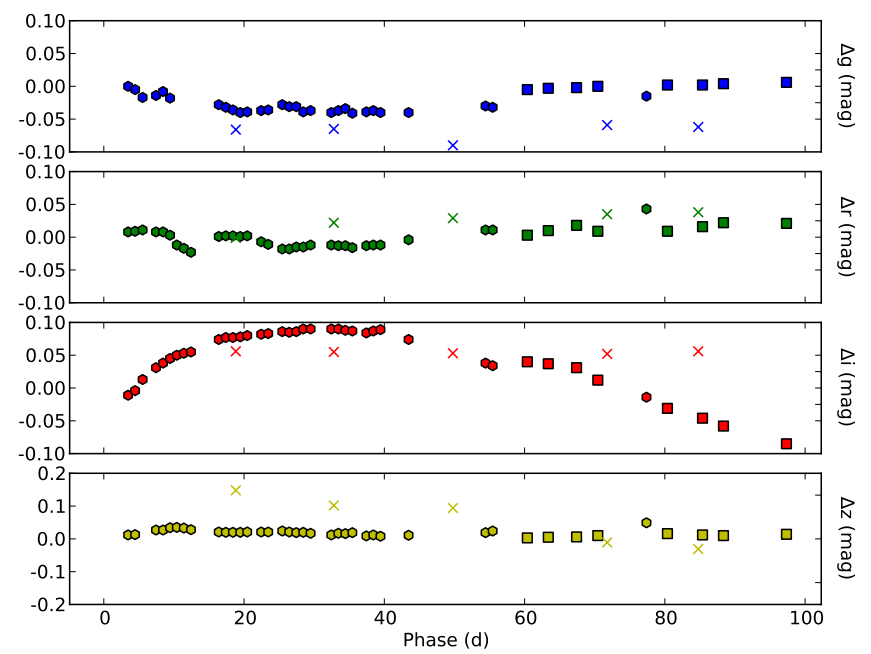

Fig. A.3. Difference between SDSS colour- and S-corrected photometry for NOT (squares), LT (circles) and FTN (crosses).

spectra with observed colour-term coefficients and the agreement was generally good. However, for those filters were the synthetic and observed colour-term coefficients deviated the most, we adjusted the response functions by small wavelength shifts (typically between 50 and $100 \AA$ ). This method is similar to the one applied by S11 and, in all cases, improved the agreement between S-corrected photometry from different telescopes. As we could not measure the 2MASS colour-term coefficients with sufficient precision this method could not be applied to the NIR filters.

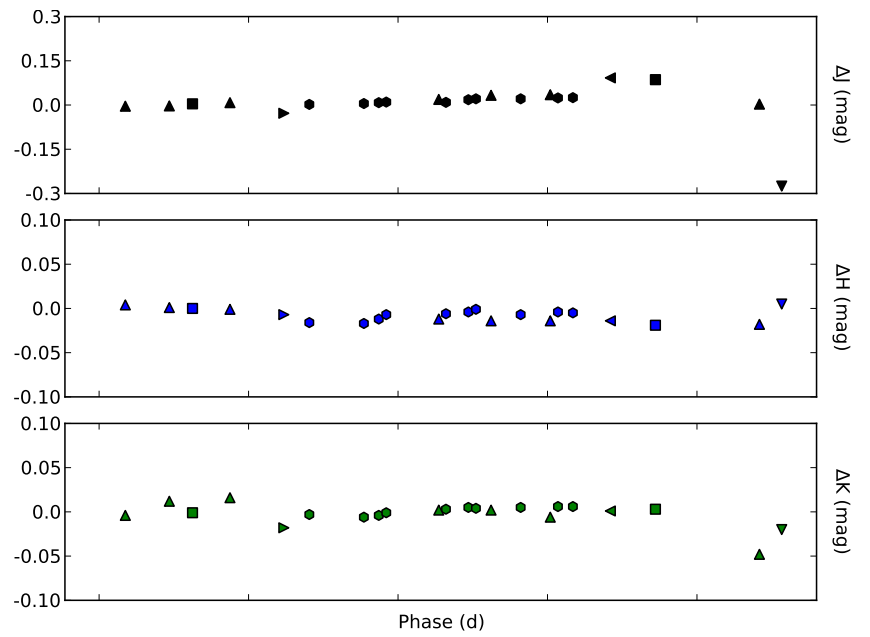

Fig. A.4. Difference between 2MASS colour- and S-corrected photometry for NOT (squares), TCS (circles), TNG (upward triangles), CA $3.5 \mathrm{~m}$ (downward triangles), WHT (rightward triangles) and LBT (leftward triangles).

\section{A.6. Systematic errors}

The difference between the S-corrected and colour-corrected JC, SDSS and 2MASS photometry is shown in Figs. A.2-A.4. The differences are mostly less than 5 percent but approaches 10 percent in some cases. Most notably, the difference for the late CA $3.5 \mathrm{~m} \mathrm{~J}$ band observation is $\sim 30$ percent because of the strong He $10830 \AA$ line. So even if the differences are mostly small, S-corrections seems to be needed to achieve 5 percent accuracy in the photometry.

A further check of the precision in the photometry is provided by comparison to the A11, V12, T12, M13, D13, S13 and, in particular, the Swift photometry. The Swift photometry was transformed to the JC system using S-corrections calculated from the Swift filter response functions, which are well known and not affected by the atmosphere. The differences between the JC photometry for all datasets and a spline fit to these observations are shown in Fig. A.5. The systematic (mean) difference for the NOT and LT datasets (which constitutes the bulk of our data) is less than $\sim 5$ percent in the $B, V, R$ and $I$ bands and less than $\sim 10$ percent in the $U$ band. Considerably larger differences, in the 15-30 percent range, are seen in some datasets, like the A11 WISE $1 \mathrm{~m} V, R$ and $I$, the M13 $B$ and $V$, the D13 $U$ and the $\mathrm{S} 13 B$. The difference between the NOT and LT and the WISE $1 \mathrm{~m}$ photometry seems to arise partly from differences in the reference stars magnitudes (Iair Arcavi, priv. comm.). As seen in Fig. A.5 as well as in Fig. 1, except for the early (0-40 days) NOT $U$ band observations, the agreement between the NOT, LT and Swift $U, B$ and $V$ band photometry is excellent and the systematic (mean) differences are only a few percent. The early (0-40 days) NOT $U$ band photometry shows a systematic (mean) 


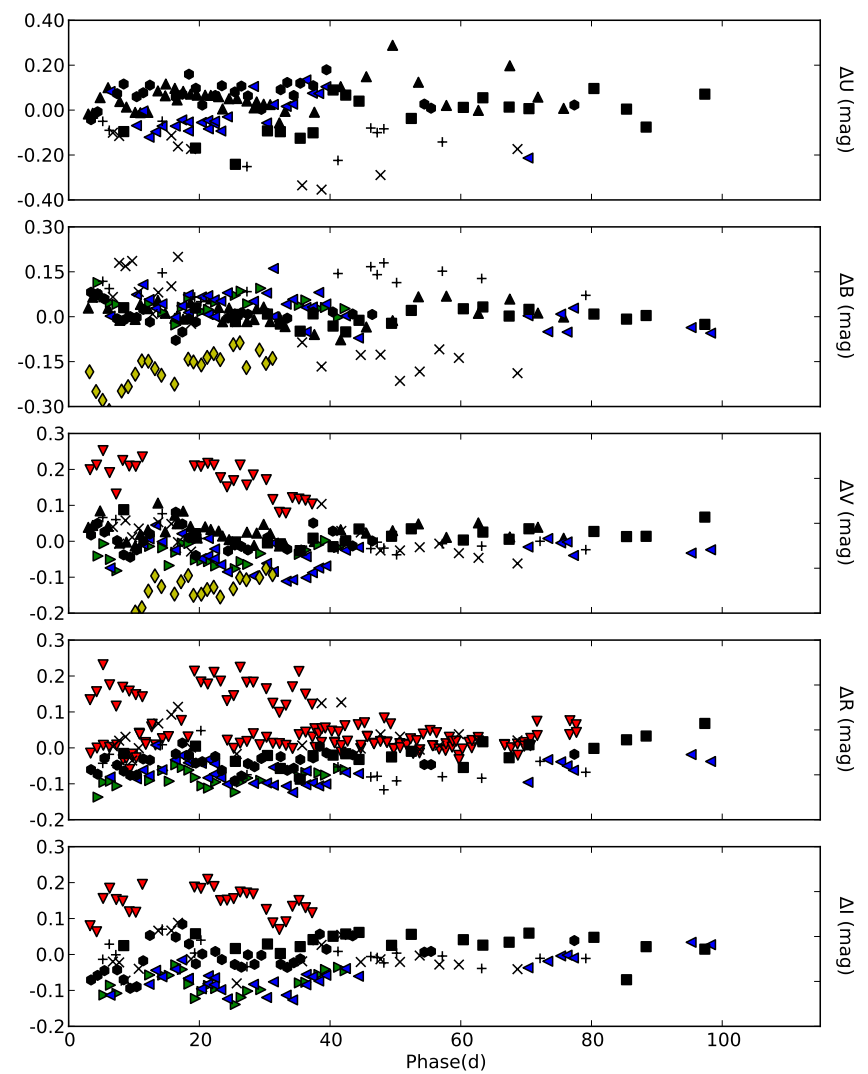

Fig. A.5. Difference between JC photometry for the NOT (black squares), LT (black circles), Swift (black upward triangles), A11 (red downward triangles), V12 (green rightward triangles), T12 (blue leftward triangles), M13 (yellow diamonds), D13 (black crosses) and S13 (black pluses) datasets and a cubic spline fit to all these datasets.

difference of $\sim 20$ percent as compared to the LT and Swift photometry which could be explained by the lack of S-corrections. As the LT filter, in spite of its SDSS nature, is more similar to the JC $U$ band than the NOT filter we favour the LT and Swift photometry in this phase. The good agreement between the NOT and LT JC photometry and the S-corrected Swift JC photometry as well as the bulk of the published JC photometry, gives confidence in the method used. The limited amount of SDSS and 2MASS observations published so far prevents a similar comparison to be done for the SDSS and 2MASS photometry.

\section{A.7. Synthetic photometry}

Synthetic photometry (used for e.g. S-corrections) was calculated as energy flux based magnitudes in the form $m_{i}=$ $\int F_{\lambda} S_{\lambda, i} \mathrm{~d} \lambda / \int S_{\lambda, i} \mathrm{~d} \lambda+Z_{i}$, where $S_{\lambda, i}$ and $Z_{i}$ are the energy response function (BM12) and zeropoint of band $i$ respectively. Note that filter response functions are commonly given as photon response functions (BM12) and then have to be multiplied with wavelength to give the energy response functions. JC filter response functions have been adopted from BM12 and zeropoints calculated using the Vega spectrum and JC magnitudes. SDSS filter response functions have been adopted from Doi et al. (2010) and zeropoints calculated using the definition of AB magnitudes (Oke \& Gunn 1983) and small corrections following the instructions given at the SDSS site. 2MASS filter response functions have been adopted from Cohen et al. (2003) as provided by the Explanatory Supplement to the 2MASS All Sky

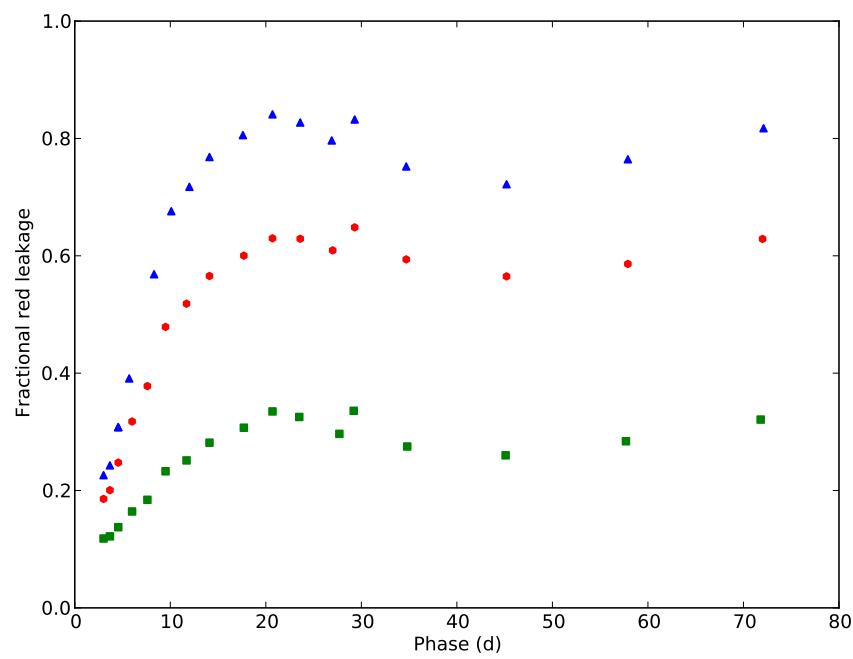

Fig. A.6. Fractional red leakage in the Swift UVW1 (red circles), UVM2 (green squares) and $U V W 2$ (blue triangles) filters.

Data Release and Extended Mission Products. Swift filter response functions have been adopted from Poole et al. (2008) as provided by the Swift calibration database. To transform the photon count based Swift system into an energy flux based system we have multiplied the response functions with wavelength and re-normalized. The zeropoints were then calculated using the Vega spectrum and Swift magnitudes (Poole et al. 2008). Spitzer filter response functions have been adopted from Hora et al. (2008) as provided at the Spitzer web site and zeropoints calculated using the Vega spectrum and Spitzer magnitudes.

\section{A.8. Swift UV read leak}

The response functions of the Swift $U V W 1$ and $U V W 2$ filters (Poole et al. 2008) have a quite strong red tail. If, as is often the case for $\mathrm{SNe}$, there is a strong blueward slope of the spectrum in the UV region this will result in a red leakage that might even dominate the flux in these filters. In Fig. A.6 we quantify this by showing the fractional red leakage defined as the fractional flux more than half the equivalent width redwards of the mean energy wavelengths of the filters. The spectrum was interpolated from the photometry as explained in Sect. 3.3 excluding the $U V W 1$ and $U V W 2$ filters. After $\sim 20$ days the leakage is $\sim 80$ and $\sim 60$ percent in the $U V W 1$ and $U V W 2$ filters respectively. Given this the $U V W 1$ and $U V W 2$ lightcurves do not reflect the evolution of the spectrum at their mean energy wavelengths and we will therefore exclude these from our analysis.

\section{Appendix B: Progenitor observations}

We have obtained high quality pre- and post-explosion $B, V$ and $r$ band images of the SN site with the NOT. The pre-explosion images were obtained on May 262008 (B) and May 292011 ( $V$ and $r$ ), the latter just 2 days before the explosion. Two sets of post-explosion images were obtained, the first on Jan. 202013 ( $V$ and $r$ ) and Mar. $192013(B), 601$ and 659 days post explosion respectively, and the second on Apr. $142013(V)$, May 152013 $(r)$ and June $12013(B), 685,715$ and 732 days post explosion respectively. In Fig. B.1 we show a colour composite of the pre-explosion and the second set of post-explosion $B, V$ and $r$ band images where the RGB values have been scaled to match 

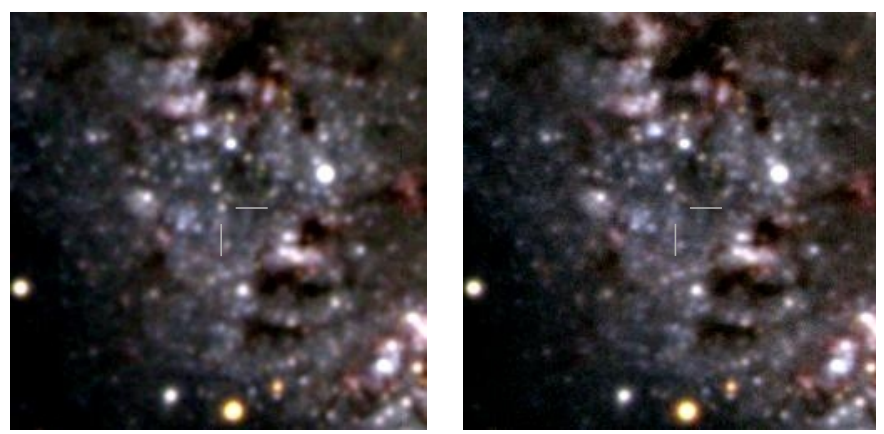

Fig. B.1. Colour composite of the pre- (left panel) and post- (right panel) explosion NOT imaging. The RGB values have been scaled to match the number of photons.

the number of photons. The photometry presented below have been calibrated to the natural Vega $(B V)$ and $\mathrm{AB}(r)$ systems of the NOT using the reference star magnitudes and colour constants presented in this paper (Tables A.5, A.7, A.2 and A.3).

We have used the HOTPANTS package to perform subtractions of the pre- and post-explosion images and aperture photometry to measure the magnitudes of the residuals to $B=$ $23.00 \pm 0.10, V=22.73 \pm 0.07$ and $r=22.22 \pm 0.05 \mathrm{mag}$ for the first set of post-explosion observations and $B=22.73 \pm 0.06$, $V=22.23 \pm 0.05$ and $r=21.95 \pm 0.04 \mathrm{mag}$ for the second set of post-explosion observations. The positions of the residuals in all bands are within 0.15 arcsec from the position of the SN. The two fainter nearby stars, seen in pre-explosion HST images, that could possibly contaminate the result are $\sim 0.5$ arcsec away from the SN so their contribution (due to variability) to the residuals is likely to be small. Using PSF photometry where we have iteratively fitted the PSF subtracted background we measure the magnitudes of the yellow supergiant in the pre-explosion images to $B=22.41 \pm 0.12, V=21.89 \pm 0.04$ and $r=21.67 \pm 0.03 \mathrm{mag}$. The residuals for the second set of post-explosion observations then corresponds to a reduction of the flux with $74 \pm 9,73 \pm 4$ and $77 \pm 4$ percent in the $B, V$ and $r$ bands respectively. The remaining flux, at least partly emitted by the $\mathrm{SN}$, corresponds to $B=23.35 \pm 0.32, V=22.56 \pm 0.10$ and $r=22.67 \pm 0.11 \mathrm{mag}$ for the first set of post-explosion observations and $B=23.89 \pm 0.50$, $V=23.32 \pm 0.20$ and $r=23.28 \pm 0.19$ mag for the second set of post-explosion observations. 